LBL- $-28 \subseteq 81$

DE90 013358

\title{
Nuclear-Decay Studies of Neutron-Rich Rare-Earth Nuclides
}

by

Robet Mark Chasteler

$\mathrm{Ph} . \mathrm{D}$. Thesis

(April 26, 1990)

Department of Chemistry

University of California, Berkeley

and

Nuclear Science Division

Lawrence Berkeley Laboratory

1 Cyclotron Road

Berkeley, CA 94720

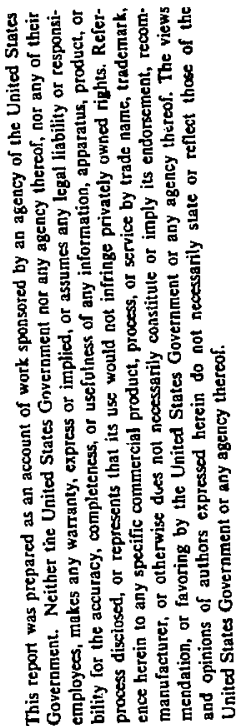

This w ork was supported by the Director, Office of Energy Research, Division of Nuclear Physics of the Office of High Energy and Nuclear Physics of the U.S. Department of Energy under Contract No. DE-AC03-76SF00098. 


\title{
Nuclear-Decay Studies of Neutron-Rich Rare-Earth Nuclides
}

\author{
Robent Mark Chasteler
}

\section{ABSTR ACT}

Neutron-rich rare-earh nuclei were produced in multinucleon transfer reartions of ${ }^{170} \mathrm{Er}$ and ${ }^{176} \mathrm{Yb}$ projectiles on narW targets at the Lawrence Berkeley Laboratory SuperHILAC and their radioactive decays properties studied at the on-line mass separation facility OASIS. Two unkrown isotopes, ${ }^{169} \mathrm{Dy}\left(\mathrm{t}_{1 / 2}=39 \pm 8 \mathrm{~s}\right)$ and ${ }^{174} \operatorname{Er}\left(t_{1 / 2}=3.3 \pm 0.2 \mathrm{~m}\right)$ were discovered and their decay characteristics determined. The decay schemes for two previously identified isotopes, ${ }^{168} \mathrm{Dy}\left(\mathrm{t}_{1 / 2}=8.8 \pm 0.3 \mathrm{~m}\right)$ and ${ }^{17} \mathrm{H}_{\mathrm{Ho}}\left(\mathrm{t}_{1 / 2}=55 \pm 3 \mathrm{~s}\right)$, were characterized. Evidence for a new isomer of $3.0 \mathrm{~m}{ }^{168} \mathrm{Hog},{ }^{168} \mathrm{Ho}^{m}\left(t_{1 / 2}=132 \pm 4 \mathrm{~s}\right)$ which decays by isomeric transition (IT) is presented. Beta particle endpoint energies were determined for the decay of ${ }^{169} \mathrm{Hog},{ }^{169} \mathrm{Dy},{ }^{171} \mathrm{Ho}$, and ${ }^{174} \mathrm{Er}$, the resulting $Q_{\beta}$ values are: $2.93 \pm 0.03,3.2 \pm 0.3,3.2 \pm 0.6$, and $1.8 \pm 0.2 \mathrm{MeV}$, respectively. These values were compared with values calculated using recent atomic mass formulae. Comparisons of various target/ion source geometries used in the OASIS mass separator facility for these muldinucleon transfer reactions were performed. 


\section{DEDICATION}

Tiis thesis is dedicated to my two best friends who supported me during the writing stage. Their words of encouragement convinced even me that I could finish it. If it wasn't for their support and encouragement, I wouldn't have received any and this theris would never have been compleied. Lauren and Lisa, I thank you both from the bottom of my heart and am indebred to you for the rest of my life. 


\section{ACKNOWLEDGEMENTS}

This work was supported by the Director, Office of Energy Research, Division of Nuclear Physics of the Office of High Energy and Nuclear Physics of the U.S. Department of Energy under Contract No. DE-AC03-76SF00098. The support of the Nuclear Science Division of Lawnence Berkeley Laboratory and the Chemistry Department of the University of California, Berkeley.

I would like to thank Prof. Darleane Hoffman for the opportunity given to me in leaming nuclear chemistry and graduate research. The first three years of my six year graduate career were spent under Darleane's guidance. Even though the research I performed during that time is not contained in this thesis, une tools and skills I learned then were invaluable in compiling this work. I would like to thank the other nembers of the LBL Heavy Ion Radiochemistry Group I worked with (D. Lee, M. Nurmia, K. Gregorich, R. Henderson, C. Gannett, D. Bennett, and R. Chadwick) for their assistance.

I arn indebted to the OASIS group (M. Nitschke, P. Wilmarth, $K$. Vierinen, R. Firestone, and A. Shihab-Eldin) for helping me perform the experiments of this thesis. The last three years of my graduate career were spent working with and leaming from these fine scienrists. A special thanks goes to Phil W. and Kari V. for their guidance and direction when I first joined the group. If it wasn't for their help I would have floundered around for at least a year and delayed this work by at least as much time. Also, I wish an extra thanks to Phil W. for the help preparing this thesis in running the Macintosh and providing some figures.

The outstanding technical support of L. Archambault and A. Wydler in keeping OASIS running whenever it was needed is acknowledged. I would also like 
to extend an extra thanks to L. Archambault for providing the CAD drawing of the OASIS ion source used in Figure 3.4. The assistance of the staff and crew of the SuperHILAC is also noted.

Last, but not least, I owe a thanks to all my friends, family, and roommates (past and present) for their friendship and support during my graduate career. I will not even try to name you all, for I will certainly forget someone. A big thank-you is sent to you all for helping keep my sanity in Berkeley. 
TABLE OF CONTENTS

1. INTRODUCTION 1

2. MULTINUCLEON TRANSFER REACTIONS 8

3. EXPERIMENTAL 14

3.1. THE SUPERHILAC 14

3.2. OASIS 16

3.2a. TARGET/ION SOURCE SYSTEMS 16

3.2b. THE SEPARATOR 21

3.2c. TRANSPORT SYSTEMS 22

3.2d. THE DETECTOR SYSTEM 23

3.3. DATA ACQUISITION 26

3.3a. DETECTOR ELECIRONICS 26

3.3b. COMPUTERS AND DATA ACQUISITION SOFTWARE 27 3.4. EXPERIMENT SPECIFICS 28

3.4a. ${ }^{176} \mathrm{Yb}$ EXPERIMENT 28

3.4b. ${ }^{170}$ Er EXPERIMENT 29

4. DATA ANALYSIS

4.1. GAMMA SINGLES DATA 30

4.2. COINCIDENCE DATA 33

4.3. BETA TELESCOPE SPECTRA

5. RESULTS 40

5.1. $A=168$ MASS CHAIN 42 
5.1a. ${ }^{168}$ Dy DECAY SCHEME

5.1b. ${ }^{168} \mathrm{Ho}^{\mathrm{m}}$ ISOMER

5.1c. ${ }^{168}$ Ho Qp-CORRECTIONS

5.2. ${ }^{169} \mathrm{Dy}$ - NEW ISOTOPE

5.3. ${ }^{171}$ Ho DECAY SCHEME

5.4. ${ }^{174} \mathrm{Er}-\mathrm{NEW}$ ISOTOPE

6. CONCLUSIONS 


\section{LIST OF FIGURES}

1.1. Fission yields from ${ }^{235} \mathrm{U}$ and ${ }^{252} \mathrm{Cf}$

1.2. Chart of nuclides for neutron-rich lanthanides 6

2.1. Cross-section calculations 12

3.1. SuperHILAC beam optics used for OASIS 15

3.2. OASIS diagram 17

3.3. Beam scanner display 19

3.4. Normal OASIS ion source 20

3.5. Detector arrangement 24

4.1. Detector efficienies 32

4.2. $A=168, \beta$ decay data and Fermi-Kurie plots with $741-\mathrm{keV} \gamma$ gate $\quad 36$

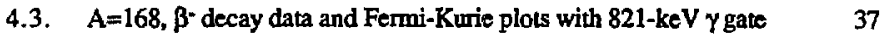

4.4. Calculated $\beta$ - spectra fits for 741- and $821-\mathrm{keV} \gamma$ gates for $A=168 \quad 39$

5.1. Chart of nuclides for region studied 41

5.2. A=168, 1024-s run, Ho K x-ray decay curves

5.3. $A=168,1024-\mathrm{s} \mathrm{run},{ }^{168}$ Ho growth and decay curves 46

5.4. ${ }^{168}$ Dy dexay scheme $\quad 48$

5.5. 168 Ho $193-\mathrm{keV}$ level decay curve 50

5.6. Ho K X-ray decay curves, $A=168,512$-s an 52

5.7. 168 Ho growth and decay curves, $A=168,512-s$ run 54

5.8. Theoretical $x$-ray intensities for ${ }^{168} \mathrm{Hom}^{\mathrm{m}}$ decay 56

5.9. ${ }^{172} \mathrm{Lu}^{\mathrm{m}}$ and ${ }^{168} \mathrm{Ho}^{\mathrm{m}}$ decay schemes 58 
5.10. ${ }^{168} \mathrm{Ho} 821-\mathrm{keV}$ level $\beta$ - decay data and Fermi-Kurie plot 61

5.11. ${ }^{168} \mathrm{Ho} 896-\mathrm{keV}$ level $\beta$ - decay data and Fermi-Kurie plot 62

5.12. ${ }^{168} \mathrm{Ho} 995-\mathrm{keV}$ level $\beta$ - decay data and Fermi-Kurie plot 63

5.13. $\beta$ decay data and Fermi-Kurie plot, for $A=169$

5.14. $1578-\mathrm{keV}$ decay curve for $A=169$ mass chain 69

5.15. $\beta$ - particles coincident with $1578 . \mathrm{keV} \gamma$ rays in the $A=169$ mass chain 69

5.16. Maximum likelihood fit of $\gamma$ coincident $\beta$ particles 70

5.17. Calculated $\beta$ - spectrum fit for $A=169$ mass chain 72

5.18. ${ }^{169}$ Dy decay scheme $\quad 73$

5.19. $\beta$ decay data and Fermi-Kurie plot for $A=171$

5.20. $\beta$-coincident $\gamma$ spectra for $A=171$ mass chain 78

5.21. ${ }^{171}$ Ho decay scheme $\quad 82$

5.22. ${ }^{171} \mathrm{Er} 199-\mathrm{keV}$ level decay curve 83

5.23. ${ }^{171} \mathrm{Ho} \beta$ - endpoint data 84

5.24. Low-energy $\gamma$ spectrum for $A=174$ mass chain 88

5.25. ${ }^{174} \mathrm{Er}$ decay scheme $\quad 89$

5.26. ${ }^{174} \mathrm{Er} \beta$ - endpoint data and Fermi-Kurie plot 91

5.27. $176 \mathrm{Yb}$ experiment $\beta$ telescope calibration data and Fermi-Kurie plot 92

5.28. Targetfion source geometries used 93 


\section{LIST OF TABLES}

5.1. Electromagnetic multipole selection rules 42

5.2. ${ }^{168}$ Dy decay data 47

5.3. ${ }^{168} \mathrm{Ho} \beta^{-}$endpoint and $Q_{\beta}$ - data 64

5.4. ${ }^{168} \mathrm{Ho} \log f$ values $\quad 64$

5.5. $A=169$ isobar decay information $\quad 67$

5.6 A=171 isobar decay information 76

5.7. ${ }^{171}$ Ho decay data $\quad 80$

5.8. ${ }^{174}$ Er decay daca $\quad 86$

5.9. Target/ion source results for ${ }^{168} \mathrm{Ho}$ production 95

5.10. Target/ion source : esults for ${ }^{168} \mathrm{Lu}$ production 96

6.1. Experimental versus theorerical Q\$-comparisons 100 


\section{INTRODUCTION}

This investigation is concemed wiih the synthesis and decay studies of neutron-rich rare-earth nucles. Nuclear-decay informaticn for neutron-rich isotopes has important applicarions to astrophysical problems and reactor technology. Neuron-binding energies and nuclear halif-lives are the most inportani parameters needed for calculations of astrophysical nucleosynthesis. Nuclear half-lives of neutron-rich isotopes are particularly critical in the case of rapid neutron capture (the $r$-process). During the $r$-process, in the enormous neutron flux inside of stars, the time required for a nucleus to capture a neutron is very short compared to beta decay times and the neutron exposure time is many times longer than the beta cecay halflives so that successive beta decays can take place during bombardment. During supernova explosions, neutron ñuxes reach high enough levels, for short periods of time. that "prompt" capture processes occur in which reutrons are quickly added to a mucleus until the product is at the neutron-drip line (point where the neutron-binding energy equals zero). Both the $r$-proc-ss and "prompt" capnure frocess result in the flow of nucleosynthesis along the neutron-drip line [Cow70]. Peaks in the mass abundance distribution ocsur around masses 80, 130, 165, and 194 where the $r$ process reaches magic neutron shells and further neutron capture is hindered due to the low neutron-binding energy. The mass flow moves along these neutron numbers because of $\beta$ - decay and subsequent neutron cipture back towards the magic nentron numbers.

With the neutron-drip line approximately 50 mass units away from the known isotopes in the rare-earth region, astrophysicists have to depend heavily on half-life predictions for their calculations. At the star of this research, two sets of half-life 
estimates for large numbers of neutron-rich nuclei existed. Takahashi, Yamada, and Kondoh [Tak73] calculated beta decay half-lives using the gross theory of beta decay. Klapdor, Metzinger, and Oda [Kla84] used calculations incorporating a detailed treatment of beta strength functions. In general, the calculations of Takahashi, Yamada, and Kondoh overestimate and the calculations of Kiapdor, Metzinger, and Oda underestimate half-lives of nuclei in the neutron-rich rare-earth region. The deviations from the experimental half-lives at times approach an onder of magninde resilting in large uncertainties in the calculation of astrophysisal nucleosynthesis.

Decay information for these neutron-rich isotopes is also important in developing and testing nuclear theory. Theoretical calculations of nuclear level energies [Ni169, Mö189] and deformations [Mö181] are used in predicting $\beta$ strength functions and ground-state spins and parities. Also numerous calculations of atomic masses have been made [M8181, Hau88 (and references therein)] with varying results. Since most of these calculations depend on measured masses, spins, and parities to determine the best parameter sets for their models, new decay information is always needed. Also, half-life prediction codes [Tak73, Kla84] are dependent on the atomic masses used.

The present investigation is concerned with the decay properties of neutronrich rare-earth nuclei with masses greater than $\mathrm{A} \sim 160$. There is very little information known about nuclei in this important region. Prior to about 1980 , nuclei of only two neutrons more or two protons less than the most neutron-rich stat: isotopes were known for this region. These were made using relatively simple reactions (i.e. $(n, \gamma),(n, \alpha),(n, p),(\gamma, p),(p, 2 p),(p, 3 p)$, and $(t, p))$ which could be performed with nuclear reactors, neutron generators, and Van de Graaff accelerators. 
After approximately 1980 , two new methods of producing neutron-rich rare-earth isotopes with $A>160$ were investigated; fission of heavy actinides and multinucleon gransfer reactions.

The fission of heavy actinides proves to be very efficient in producing new neutron-rich isotopes of the rare-earth elements from $\mathrm{Ce}(\mathrm{Z}=58)$ to $\mathrm{Tb}(\mathrm{Z}=65)$ [Mac86, Gre87, Kaw88, and references therein]. Thermal neutron fission of ${ }^{235} \mathrm{U}$ has been used at Brookhaven National Laboratory (BP.T.) to produce isotopes with masses up to $\mathrm{A}=161$ [Mac86]. However, their research is limited by the mass yields from thermal neutron fission of ${ }^{235} \mathrm{U}$ which drops drastically above mass $\mathrm{A}=160$ (Figure 1.1). Researchers at Idaho National Engineering Laboratory (INEL) have used the spontaneous fission of ${ }^{252} \mathrm{Cf}$ as a production source of neutron-rich rareearth nuclei [Gre87]. The extra 16 nucleons in ${ }^{252} \mathrm{Cf}$ as compared to $235 \mathrm{U}+$ nthemal, shifts the heavy mass tail up about 10 atomic mass units as seen in Figure 1.1. This shift has allowed INEL to investigate neutron-rich rare-earth isotopes up to mass $A=168$ [Geh82].

In the early 1980's, researchers at Gesellschaft für Schwerionenforschung (GSD) started using multinucleon transfer reactions to produce new neutron-rich nuclei of heavy rare-earths and nuclei lighter than the group of light fission products ( $A=52-79$ ) [Run85]. In the case of heavy rare-earths, natw/Ta or 186W/Ta targets were bombarded with $136 \mathrm{Xe}$ and ${ }^{238} \mathrm{U}$ ions at $11.5 \mathrm{MeV} / \mathrm{u}$ [Ryk89]. Since ${ }^{136} \mathrm{Xe}$ is an $\mathrm{N}=82$ nuclide with six stable $\mathrm{N}=82$ stable isotones with atomic numbers greater than $54(\mathrm{Xe})$, one expects some preference ior the pick-up of protons from the target nucleus. The increased $\mathrm{N} / \mathrm{Z}$-ratio of the ${ }^{238} \mathrm{U}$ projectile should enhance the production of neutron-rich nuclei compared with lighter projectiles. Both projectiles, ${ }^{136} \mathrm{Xe}$ and ${ }^{238} \mathrm{U}$, have been successful in producing nieutron-rich rare-earth nuclei 


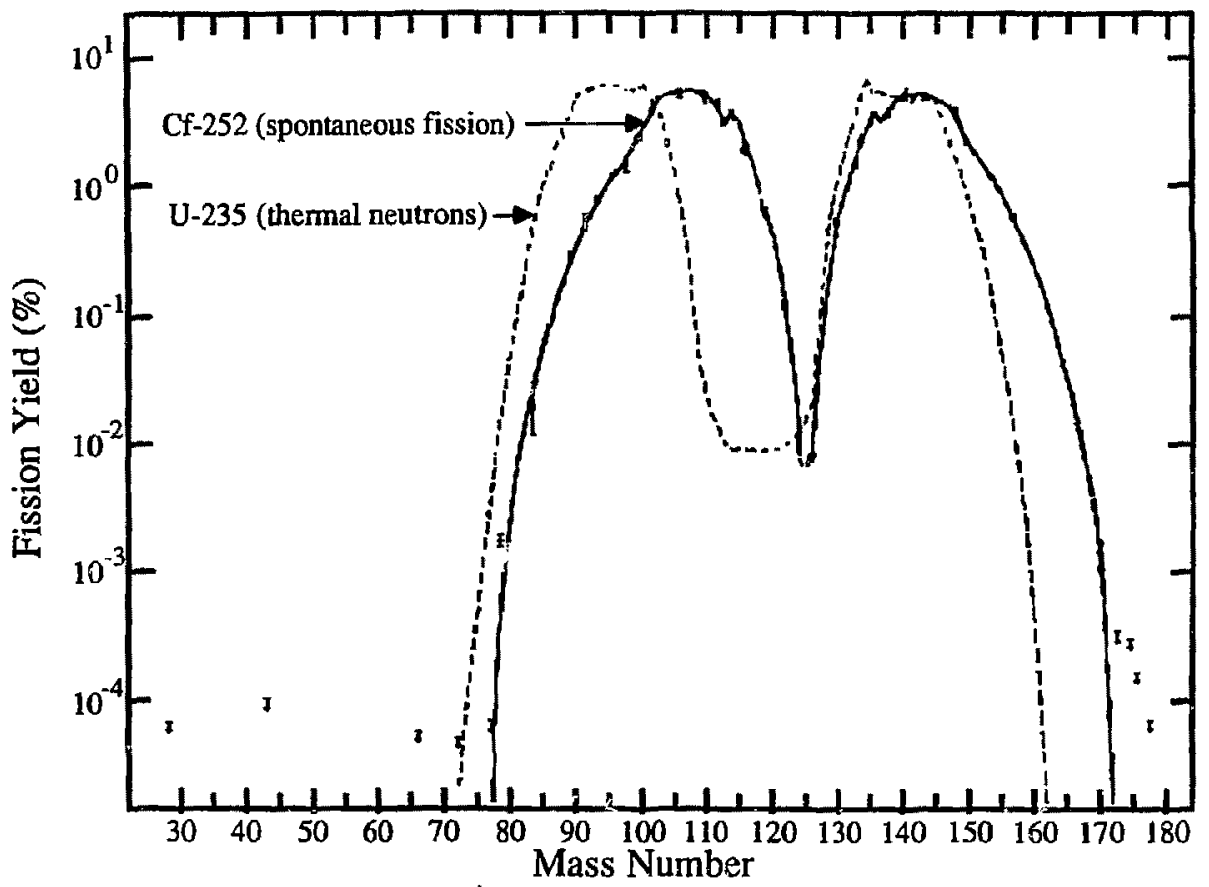

Figure 1.1.Spontaneous fission yield from $\mathrm{Cf}-252$ as compared with the yield curve for the thermal neutron fission of U-235. Curves are taken from Hyde [Hyd71]. 
having masses $A=177-184$ with similar production yields [Ryk89]. 136Xe has proven to be the only projectile to produce the lighter masses $A=171,172$, and 174 [Ryk89, Mei89]. For the production of nuclei below the group of light fission products [Run85], a different method of isotope production was used. Because GSI's mass separator ion source requires heavy refractory materials, like W or Ta, for non-compound nucleus reactions, arget-like fragments in the $A \sim 65$ region can not be produced. However, using neutron-rich beams, near the desired nuclei, on (nat or 186)W/Ta targets, neutron-rich projectile-like fragments can be produced. In these reactions, the target picks up protons from the projectile, though, not with the same affinity as ${ }^{136} \mathrm{Xe}$. Researchers at GSI has used ${ }^{76} \mathrm{Ge}$ and ${ }^{82} \mathrm{Se}$ beams : produce ${ }^{60} \mathrm{Cr},{ }^{61} \mathrm{Mn},{ }^{64} \mathrm{Fe},{ }^{65} \mathrm{Co},{ }^{69} \mathrm{Ni}$, and $73 \mathrm{~m} \mathrm{Zn}$ [Run85, Bos88] using this method of projectile-like fragment production.

In the current investigation, multinucleon transfer reactions were used to produce new neutron-rich rare-earth nuclei. However, instead of looking at the target-like fragments in this region as did researchers at GSI, projectile-like fragments of ${ }^{170} \mathrm{Er}$ and ${ }^{176} \mathrm{Yb}$ [Cha89] beams were produced as was done at GSI in the $A=60-73$ region with ${ }^{76} \mathrm{Ge}$ and ${ }^{82} \mathrm{Se}$ beams. Using the OASIS facility [Nit83] on-line at the Lawrence Berkeley Laboratory SuperHILAC for mass separation and detection of the products, two new isotopes, ${ }^{169} \mathrm{Dy}$ and ${ }^{174} \mathrm{Er}$, and one new isomer, ${ }^{168} \mathrm{Hom}^{\mathrm{m}}$, were identified. Also, the decay schemes for two previously identified isotopes, ${ }^{168} \mathrm{Dy}$ and ${ }^{171} \mathrm{Ho}$ (one at INEL and one at GSI, respectively), were determined. Figure 1.2 shows the region of the char of the nuclides studied with the various nuclei produced at GSI, BNL, INEL, and OASIS marked. As can be seen in the figure, this research fits between the upper limits at DNEL and the lower limits at GSI. 


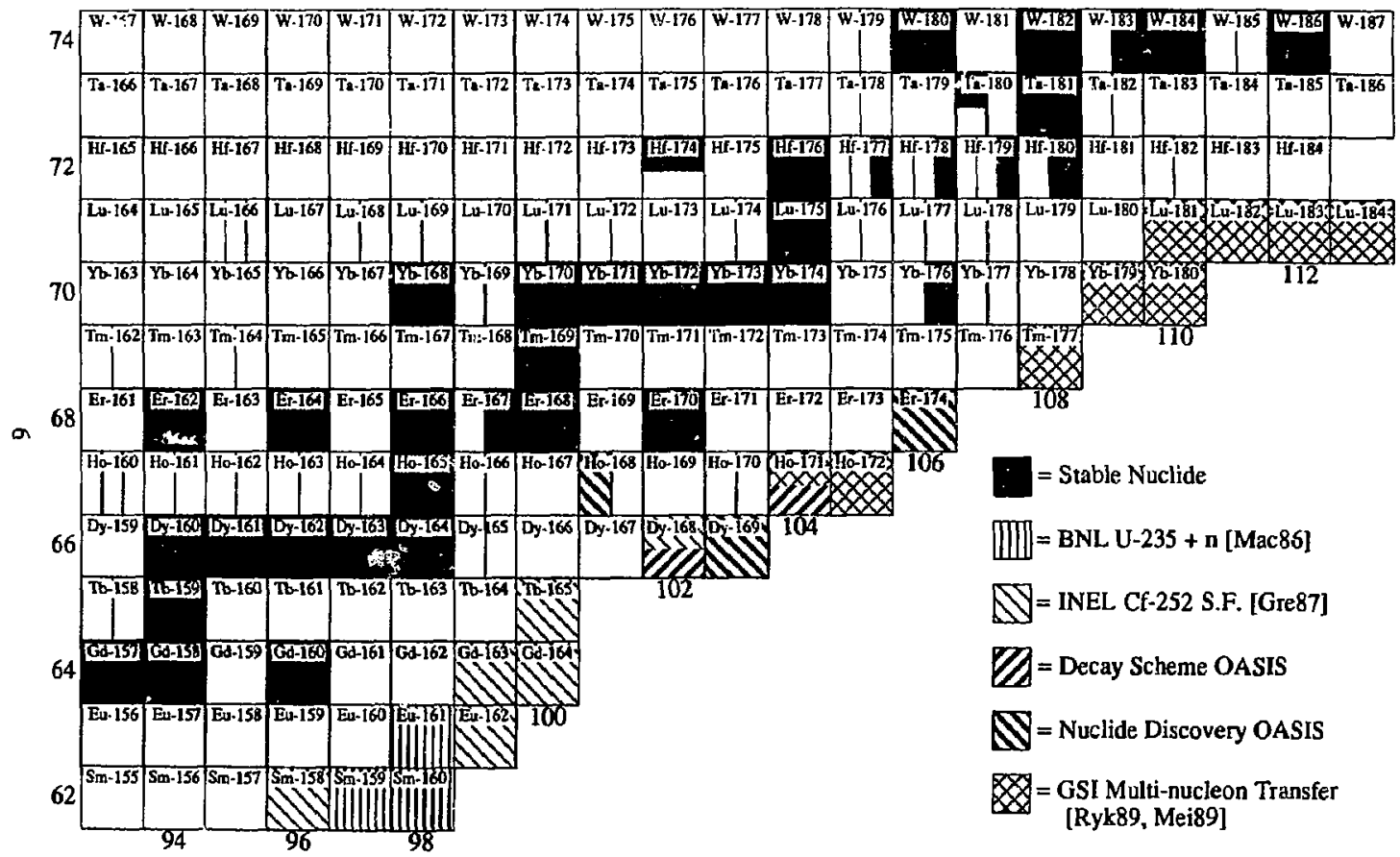

Figure 1.2. Region of the chart of nuclides showing the various nuciei produced in this study (OASIS) and at Gesellschaft für Schwerionenforschung (GSI), Idwho National Engineering Laboratory (INEL), and Brookhaven National Laboaratory (BNL). 
A short discussion of the physics of multinucleon transfer reactions and their application to this study is presented in chapter 2 . A description of the experimental conditions and parameters is given in chapter 3. A brief discussion of the data analysis techniques and computer programs used is presented in chapter 4 . The results of the experiments are presented in chapter 5. These results will be presented in order of increasing mass number rather than the chronological order in which they were produced. Conclusions from this study are discussed in chapter 6. 


\section{MULTINUCLEON TRANSFER REACTIONS}

Some of the mechanisms and properties of multinucleon transfer reactions and their application to this research study are discussed in this chapter. Heavy-ion nuclear reactions can be broadly classified into five types of reactions: "distant", "touching", "solid contact", "deeply penetrating", and "fusion" collisions [Sch77a]. "Distant" collisions, elastic scattering and Coulomb excitation reactions, and "touching" collisions, inelastic scarering and few nucleon transfer reactions, are classified as reactions with little or no energy damping (loss of kinetic energy between the entrance and exit channels). "Fusion" collisions, compound nucleus reactions, are associated with full energy damping. "Solid contact" collisions, fragmentation and multinucleon transfer reactions, and "deeply penetrauing" collisions, deep inelastic reactions, are classified as reactions with large, but not complete, energy damping. These last two reaction types are of interest to this study, where penetration depths are deen enough to allow substantial mass transfer, yet not so deep as to have complete loss of projectile and target identities. Mechanisms and properties of these damped heavy-ion collisions will be discussed below.

In strongly damped reactions, where several hundreds of MeV of kinetic energy can be lost, numerous reaction channels are energetically possible and there is no obvious preference for a binary reaction mode. Yet, in-plane kinematic coincidence of correlated fragments from reactions of ${ }^{84} \mathrm{Kr}(600 \mathrm{MeV})$ on ${ }^{209} \mathrm{Bi}$ indicate a preference for a binary reaction mode. The experimental results were consistent with a binary fragmentation of the intermediate system followed by neutron evaporation [Sch77a]. Studies using lighter heavy-ions, ${ }^{12} \mathrm{C},{ }^{16} \mathrm{O},{ }^{20} \mathrm{Ne}$, ${ }^{32} \mathrm{~S},{ }^{35} \mathrm{Cl},{ }^{40} \mathrm{Ar}$, and ${ }^{63} \mathrm{Cu}$, also lend support to the two-body nature of strongly 
damped reactions. There are indications that sequential fission of the heavy fragment is an important decay mode for reactions involving very heavy targets [Sch77a].

Assuming a binary reaction mechanism, damped heavy-ion reactions can be broken into a four-step process: formation of the dinuclear complex; charge, mass and energy exchange in the dinuclear complex; break-up of the dinuclear complex; and deexcitation of the product fragments. Evidence of the existence of the dinuclear complex has been found in reactions of ${ }^{209} \mathrm{Bi}+136 \mathrm{Xe}(1130 \mathrm{MeV})$ [Sch76, Sch77a]. Double-differential cross-section contour plots, with respect to lab angle and total kinetic energy of the products, produced a ridge that moved down in energy at an almost constant angle. This indicates strong angular focusing of an infermediate double nucleus which decays at approximately the same angle, independent of angular momentum [Sch77a]. The charge, mass, and energy exchan 8 in the dimclear complex becomes the important step in determining the products of damped heavy-ion reactions. It has been found for reactions of ${ }^{84} \mathrm{Kr}$ ions on ${ }^{165} \mathrm{Ho}$ and

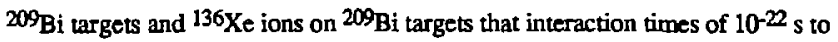
several times $10^{-21} \mathrm{~s}$ were obtained for the dinuclear complex of damped collisions [Sch77b]. Relaxation times for equilibration of the $N / Z$ distribution, kinetic energy damping, and charge distribution of dinuclear complexes have been measured as approximately $1.3 \times 10^{-22} \mathrm{s,} 3 \times 10^{-22} \mathrm{~s}$, and $60 \times 10^{-22} \mathrm{~s}$ respectively [Mor81]. A relaxation time of the $\mathrm{N} / \mathrm{Z}$ distribution on the order of $10^{-22} \mathrm{~s}$ has also been measured for Xe projectiles on Au targets [Kra79]. It can be seen from these times that the $N / Z$ distribution and kinetic energy damping are almost completely equilibrated in the dinuclear complex, while the charge distribution is not equilibrated.

Much research has been performed on the dependence of the differential cross section on bombarding energy, charge, mass, product angle, and energy damping. It 
has been found that the lab-frame angular distribution for reactions of ${ }^{84} \mathrm{Kr}$ on ${ }^{208} \mathrm{~Pb}$ exhibits a strong peak that moves forward and sharpens with increasing bombarding energy [Yan76]. In reactions of ${ }^{209} \mathrm{Bi}$ with $1130-\mathrm{MeV}{ }^{136} \mathrm{Xe}$ ions, the differential cross section was very strongly peaked at an angle of $29.8^{\circ}$ in the laboratory frame, inside of the measured quarter-point angle of $33.1^{\circ}$ [Sch76]. There was essentially no recoil intersity at $\theta_{l a b}=0^{\circ}$. The width of the angular distribution for projectilelike fragments for the above reaction increases substantially with larger energy damping [Sch77a]. The width of the charge distribution also increases with larger energy damping [Sch76]. The last two facts indicate that large mass transfers are associated with a large degree of energy damping and wide angular distributions. Measurements of the most probable mass number for each product $Z$ in reactions of

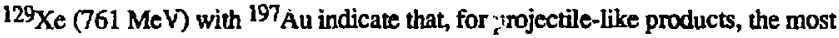
probable mass number decreases by $\sim 2$ for each $Z$ below the projectile charge ( $A_{\text {prob }}=129$ for $Z=54(\mathrm{Xe}$ ) ) [Kra79]. This indicates that the $N / Z$ equilibration tends to follow the line of beta stability.

The results mentioned above indicate that the desired products from these damped heavy-ion reactions rocoil with non-zero lab frame angles centered close to the quarer-point angle. For large energy damping, associated with large mass transfers, the angular distribution becomes quite wide. These large angles presented problums for performing these reactions using the OASIS mass separator, since previous ion sources were designed for compound-nucleus reactions. Further discussion of this problem will be presented in the experimental and resulis chapters. The quoted results indicate that large energy damping is desirable for increasing the yield of projectile-like products with a fow (2-4) protons removed. These studies uid not address the problem of the disposition of this lost kinetic energy. The energy 
loss is assumed to appear as excitation energy of the recoiling products. The normal modes of deexcitation for this region would be by neutron evaporation, or gamma emission for excitation energies below the last neutron binding energy. This hinders this research since neutron-rich nuclei are of interest $\mathrm{A}$ balance berween maximizing the width of the charge distribution, i.e. increasing the energy damping, and minimizing the amount of recoil excitation, i.e. decreasing the energy damping, is desired.

A modeling code was sought that would help determine the optimum target, projectile, and beam energy to use for these reactions and also serve as a guide for possible nuclei which could be studied. A computer code originally used to predict cross sections for ${ }^{136} \mathrm{Xe}+{ }^{249} \mathrm{Cf}$ reactions [Gru85] was used. This code assumed that the unequilibrated charge distributions took on tie features described by Wollersheim [Wol84]. After calculating the $\partial^{2} \sigma \partial 2 \partial E$ distribution [Wol84], the neutrons and excitation energy were distribured among the psuaucts. Since equilibration of the $N / Z$ distribution and kinetic energy is fast compared to the interactions times, a statistical procedure based on the calculated level densities of the dinuclear complex and all possible exit channels was used. This would yield the triple differential cross section distribution $\partial^{3} \sigma / \partial Z \partial \mathrm{N} \partial \mathrm{E}^{*}$, where $\mathrm{E}^{*}$ is the excitation energy of the target-like product. This distribution was then deexcited by neutron emission, the fission deexcitation channel of the original code being neglectcd for this region. The original code, designed for target-like product calculations, was modified to calculate projectile-like products. Figure 2.1 shows some sample crosssection calculations for ${ }^{170} \mathrm{Er}+$ narW using the modified computer code. The cross sections are listed for beam energies $10 \%, 20 \%$, and $50 \%$ above the Coulomb barrier. Cross sections were calculated for ${ }^{170} \mathrm{Er}$ projectiles on the various stable W 


\begin{tabular}{|c|c|c|c|c|c|c|}
\hline $\mathrm{Er}-168$ & \multirow{4}{*}{$\begin{array}{c}\text { Er-16 } \\
10 \%- \\
11.0 \mathrm{mb} \\
20 \%- \\
12.5 \mathrm{mb} \\
50 \%- \\
13.8 \mathrm{mb}\end{array}$} & \multirow{4}{*}{$\mathrm{Er}-\mathrm{T} / 0$} & \multirow{4}{*}{$\begin{array}{c}\text { EF- T7T } \\
10 \%- \\
3.46 \mathrm{mb} \\
20 \%- \\
3.18 \mathrm{mb} \\
50 \%- \\
3.45 \mathrm{mb}\end{array}$} & \multirow{4}{*}{$\begin{array}{c}\mathrm{Er}-172 \\
10 \% \\
1.61 \mathrm{mb} \\
20 \% \\
1.39 \mathrm{mb} \\
50 \%- \\
1.43 \mathrm{mb} \\
\end{array}$} & \multirow{4}{*}{$\begin{array}{c}\text { Er-173 } \\
10 \%- \\
229 \mu \mathrm{b} \\
20 \% \\
235 \mu \mathrm{b} \\
50 \% . \\
237 \mu \mathrm{b}\end{array}$} & \multirow{4}{*}{$\begin{array}{c}\mathrm{Er}-1 \mathrm{~T} \\
10 \%- \\
47.6 \mu \mathrm{b} \\
20 \%- \\
51.8 \mu \mathrm{b} \\
50 \%- \\
51.8 \mu \mathrm{b} \\
\end{array}$} \\
\hline & & & & & & \\
\hline & & & & & & \\
\hline & & & & & & \\
\hline Ho-167 & Ho-168 & Ho-169 & Ho-170 & Ho-171 & 110-172 & 106 \\
\hline $\begin{array}{c}10 \%- \\
8.64 \mathrm{mb} \\
20 \%- \\
9.43 \mathrm{mb} \\
50 \%- \\
9.38 \mathrm{mb}\end{array}$ & $\begin{array}{c}10 \%- \\
3.25 \mathrm{mb} \\
20 \%- \\
3.50 \mathrm{mb} \\
50 \%- \\
3.42 \mathrm{mb}\end{array}$ & $\begin{array}{c}10 \%- \\
1.70 \mathrm{mb} \\
20 \%- \\
1.85 \mathrm{mb} \\
50 \%- \\
1.77 \mathrm{mb}\end{array}$ & $\begin{array}{c}10 \%- \\
374 j \mathrm{bb} \\
20 \%- \\
411 \mu \mathrm{b} \\
50 \%- \\
370 \mu \mathrm{b}\end{array}$ & $\begin{array}{c}10 \%- \\
97.3 \mu \mathrm{b} \\
20 \%- \\
107 \mu \mathrm{b} \\
50 \%- \\
99.5 \mu \mathrm{b}\end{array}$ & $\begin{array}{c}10 \% \\
12.1 \mu \mathrm{b} \\
20 \%- \\
13.8 \mu \mathrm{b} \\
50 \%- \\
12.5 \mathrm{ub}\end{array}$ & \\
\hline $\begin{array}{c}\mathrm{Dy}-166 \\
10 \%- \\
1.09 \mathrm{mb} \\
20 \%- \\
1.02 \mathrm{mb} \\
50 \%- \\
0.873 \mathrm{mb}\end{array}$ & $\begin{array}{c}\mathbf{D y}-167 \\
10 \%- \\
261 \mu \mathrm{b} \\
20 \%- \\
241 \mu \mathrm{b} \\
50 \%= \\
208 \mu \mathrm{b}\end{array}$ & $\begin{array}{c}\text { Dy-168 } \\
10 \%- \\
77.4 \mu \mathrm{b} \\
20 \%- \\
70.1 \mu \mathrm{b} \\
50 \%= \\
59.3 \mu \mathrm{b}\end{array}$ & $\begin{array}{c}\mathrm{Dy}-169 \\
10 \%- \\
9.87 \mu \mathrm{b} \\
20 \%- \\
8.83 \mu \mathrm{b} \\
50 \%- \\
7.55 \mu \mathrm{b}\end{array}$ & 104 & & \\
\hline $\begin{array}{c}\mathrm{Tb}-165 \\
10 \%_{0} \\
79.6 \mu \mathrm{b} \\
20 \%- \\
53.6 \mu \mathrm{b} \\
50 \%_{0}- \\
35.6 \mu \mathrm{b} \\
\end{array}$ & $\begin{array}{c}\mathrm{Tb}-166 \\
10 \% \\
12.5 \mu \mathrm{b} \\
20 \%- \\
8.29 \mu \mathrm{b} \\
50 \%^{-} \\
5.41 \mu \mathrm{b} \\
\end{array}$ & 102 & & & & \\
\hline
\end{tabular}

Figure 2.1. Cross-section calculations [Gre85] for Er-170 projectiles (at energies $10 \%, 20 \%$, and $50 \%$ above the Coulomb barrier) on W(natural) target:s. 
isotopes arid then added together usizg the isotopic abundances as weighting factors to give the numbers in Figure 2.1. There is a smooth energy dependence of the $\mathrm{Er}$ and Ho cross sections, while for Dy and $\mathrm{Tb}$ isotopes the cross sections drop off rapidly with larger beam energies, and hence larger energy damping, as expected. Zycor et al. [Zyc83] measured yields for isotupes of hafnium and lutetium isotopes from reactions of ${ }^{40} \mathrm{Ar},{ }^{84} \mathrm{Kr}$, and ${ }^{136} \mathrm{Xe}$ beams on thick natW targets and found them to be similar to cross sections from thin targets for projectiles with energies of $\sim 1.3$ times the Coulomb barrier.

In the current study, producrion of the strongest sources of new neutron-rich isotopes was desired. Selection of the most neutron-rich projectiles enhances the production of neutron-rich projectile-like products. The criculations predicted that ${ }^{170} \mathrm{Er}$ and $176 \mathrm{Yb}$ were the best projectiles and these were chosen as the bombanding particles to produce neutron-rich isotopes of Dy, Ho, and Er. Target selection was limited to either Ta or W due to ion source construction, and the modeling code favored $W$. The question of optimum beam energy to use was actually a simple problem. The thickxess, of the $\mathrm{W}$ anode endplate that served as the target, 1471 $\mathrm{mg} / \mathrm{cm}^{2}$, was far thicker than the thickness required to degrade the energy of the 8.5 $\mathrm{MeV} / \mathrm{u},{ }^{176} \mathrm{Yb}$ or ${ }^{170} \mathrm{Er}$ ion beams (from the SuperHILAC) below the Coulomb barriers for interactions with $W$ of $\sim 12-14 \mathrm{mg} / \mathrm{cm}^{2}$ thickness. The cross-section calculations for all the desired isotopes showed at most a 50\% decrease in cross sections (for the Dy isotopes) at the full beam energy versus $10 \%$ above the Coulomb barrier. Therefore the maximum SuperHIL_AC beam energy, $8.5 \mathrm{MeV} / \mathrm{u}$, was chosen to ircrease the amount of target encountered by the besm at energies above the barrier. 


\section{EXPERIMENTAL}

The neutron-rich nuclei studied in this research were produced at the Lawrence Berkeley Laboratory's SuperHILAC which provided the ${ }^{170} \mathrm{Er}$ and ${ }^{176} \mathrm{Yb}$ ion beams. A few specifics about this machine will be presented in section 3.1. The OASIS mass separator, including the devices used to produce and ionize, mass separate, and transport the desired nuclei, and the detector system used to observe their decays, will be discussed in section 3.2. The various electronics, computers and other data acquisition handware and software used during the experiments will be presented in section 3.3. The final section will explain the specific experimental parameters for each experiment.

\subsection{THE SUPERHIILAC}

The SuperHILAC at the Lawnence Berkeley Laboratory delivered ion beams of $170 \mathrm{Er}$ and ${ }^{176} \mathrm{Yb}$ to the OASIS mass separawr located in Cave N, see Figure 3.1, of the experimental area. Full energy heams $(-8.5 \mathrm{MeV} / \mathrm{u})$ were used to maximize the amount of target material the beam would encounter before being decelerated below the Coulomb barrier. The beam-line optics and OASTS orientation used in this study are shown in Figure 3.1. The E42 beam-line can deliver full-rigidit beams as heary as ${ }^{238} \mathrm{U}$ into Cave $\mathrm{N}$ and OASIS. The new ieam apertures were of sufficient size to deliver two charge states simultaneously (probably $33^{+}$and $34^{+}$) of the $170_{E_{T}}$ beams on target inside of OASIS. 


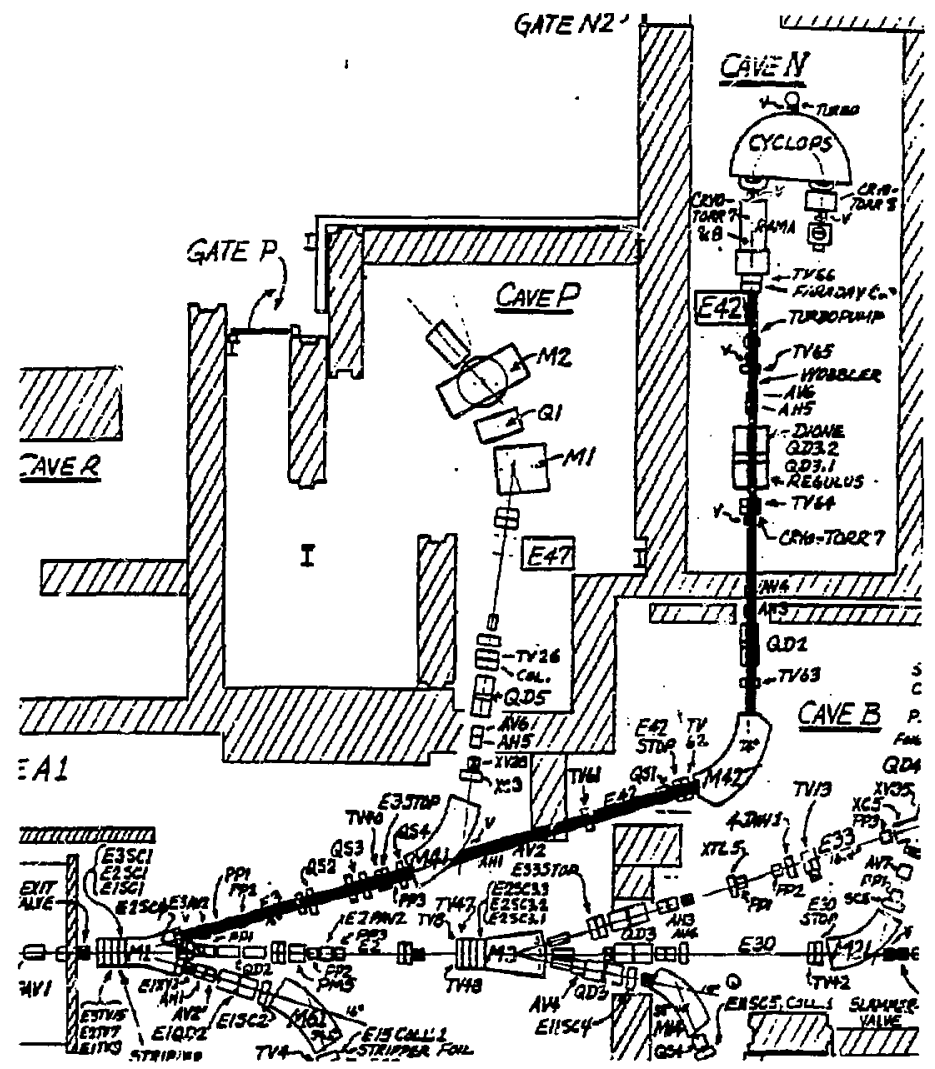

Figure 3.1. Beam optics used at the SuperHILAC for this research study. The path of the ions between bending magnets from the end of the accelerator tc OASTS is shaded. 


\subsection{OASIS}

The On-Line Apparatus for SuperHILAC Isotope Separation [Nit83] consists of four main parts discussed in the following sections: target/ion source system, the separator, transport systems, and the detector system. A modified diagram [Wil88] of the complete system is shown in Figure 3.2.

\section{2a. TARGEI/ION SOURCE SYSTEMS}

The SuperHILAC beam was transported via standard ion optical elements to the OASIS "cave". There, the beam encountered a "wobbler" (a three phase motor with the beam-line replacing the rotor) that moved the beam spot uniformly on the target to allow even heating. This "wobbler" was locared $~ 1-1 / 3$ meters upstream of the OASIS ion source. About six inches downstream from the "wobbler", a beam scanner was located for the ${ }^{170} \mathrm{Er}$ experiments. This scanner consisted of a helical wire perpendicular to the beam-line, that when rotated about the helix axis, would cause the wire to fist scan across the beam vertically and then horizontally. By measuring the current on the wire and using a digital oscilloscope for display, both horizontal and vertical beam profiles could be obtained without interrupting the beam. This information, along with electrometer readings from the collimato: and an RF pickup electrode (to measure the beam intensity entering the ion source) close to the target, determined the path of the ions into the OASIS ion source. In the case of the $170 \mathrm{Er}$ ion beams, two distinct peaks were seen in the horizontal scan, while only one was seen in the vertical scan. This can be explained assuming that two charge stares of the ${ }^{170} \mathrm{Er}$ ion beam left the $\mathbf{M 4 2}$ bending magnet (Figure 3.1) with slightly different trajectories. The beam-opics downstream of M42 were tuned to place the 


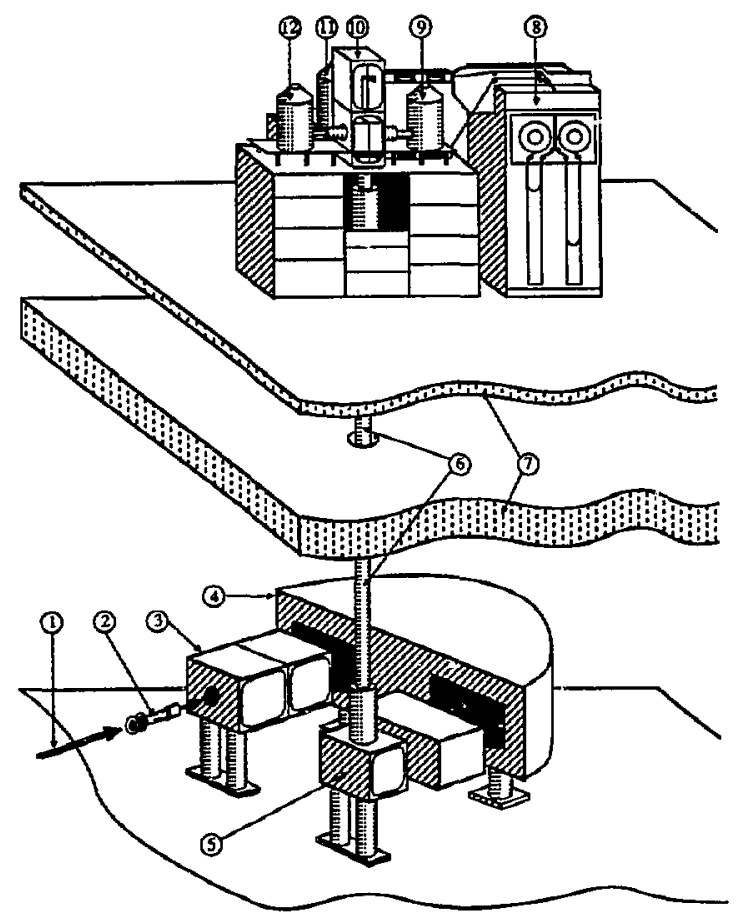

1. SuperHILAC Beam

2. Target/lon Source

3. Extraction and Focusing

4. Analyzing Magnet

5. Electrostatic Mirror

6. Transfer Line
7. Concrete Shielding

8. Tape Drive (IBM 729)

9. Ge Detector (52\%)

10. Detector Box

11. Ge Detector (24\%)

12. HPGe Detector

Figure 3.2. Simplified representation of the OASIS mass separator on-line at the LBL SuperHILAC. The major components are labeled. Figure is a modified diagram from Wilmarth [Wil88]. 
valley between the two peaks of the horizontal scan on the beam-line center of the scanner, while simulcaneously producing the largest beam-to-collimator signal racio. Figure 3.3 shows a graphic representation of the digital oscilloscope display obtained while two charge states of ${ }^{170} \mathrm{Er}$ ions were delivered to OASIS.

The normal target/ion source used in OASIS for compound nucleus reactions is shown in Figure 3.4. Due to the large recoil angles for the multinucleon transfer reactions performed in this study, the normal target position with the small $\mathrm{Ta}$ capillary tubes (22.23 $\mathrm{mm}$ in length with $\sim 1 \mathrm{~mm}$ inner diameter) would not allow the recoil products to enter the surface ionization region of the ion source. The capillaries are used to allow compound-nucleus products (small recoil angles) to enter the ionization region, but limit the amount of products diffusing back upstream to the cooler regions of the ion source. In this study, the problem of the large recoil angles and the extreme temperature $(-2500 \mathrm{C})$ in the ionization region of the ion source coupled with the desire to not have to redesign the entire ion source allowed very few solutions. The Havar window, $\mathrm{N}_{2}$ cooling gas, and compound-nucleus target were removed and the beam was allowed to pass through the carbon heat shields and the Ta capillaries and impinge upon the anode endplate. The anode endplate was used as the target and the Ta material which normally acted as the recoil catcher was replaced with W since the large number of neutrons in W compared to Ta should enhance production of neutron-rich products. This was the standard targetrion source geometry used in these multi-nucleon transfer reactions. A few ocher target/ion source geometries were tried in hopes of increasing the yield of the products. These included using a Ta endplate with a thin layer $\left(-20 \mathrm{mg} / \mathrm{cm}^{2}\right)$ of evaporated $W$, since Ta shows better diffusion of ions than $W$, or installing a thin $\left(-12-17 \mathrm{mg} / \mathrm{cm}^{2}\right) \mathrm{W}$ window/targets at the end of the capillary section with and without capillaries. 


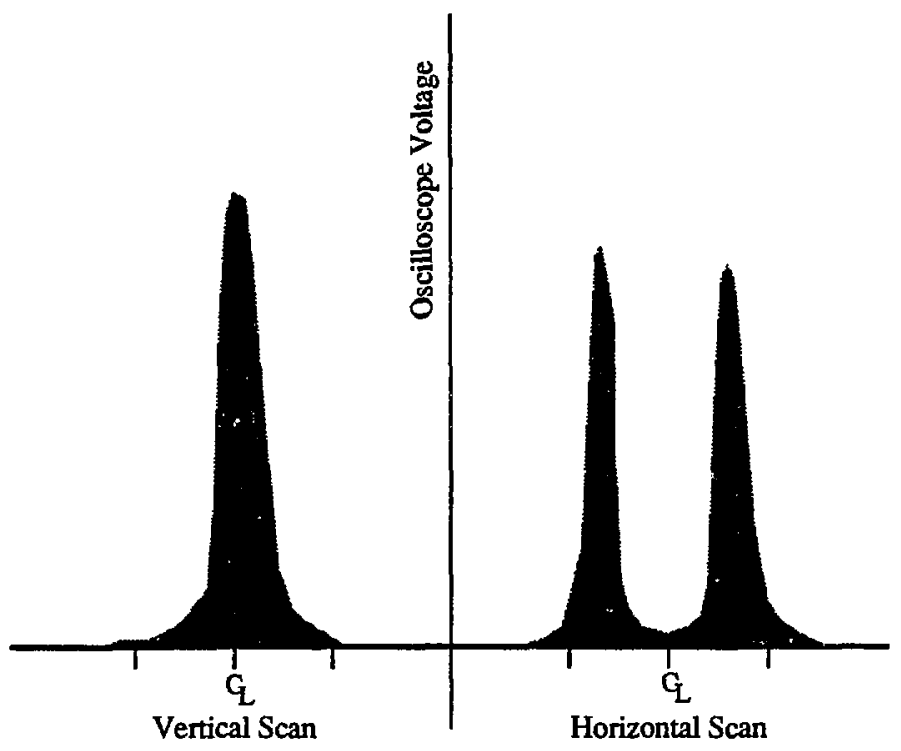

Figure 3.3. Graphic representation of the oscilloscope display for the beam scanner obrained while two charge states of $\mathrm{Er}-170$ where being delivered to OASIS. The beam-line center for each scan is marked. The extra marks on each side of the center are for $1 \mathrm{~cm}$ off the beam-line center. 


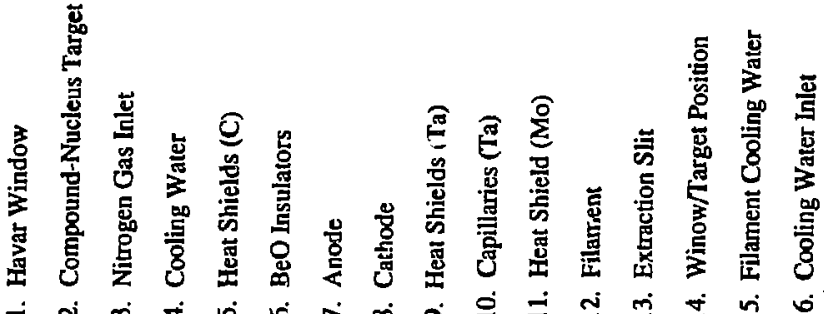

$$
\begin{aligned}
& \theta \text { (1) }
\end{aligned}
$$

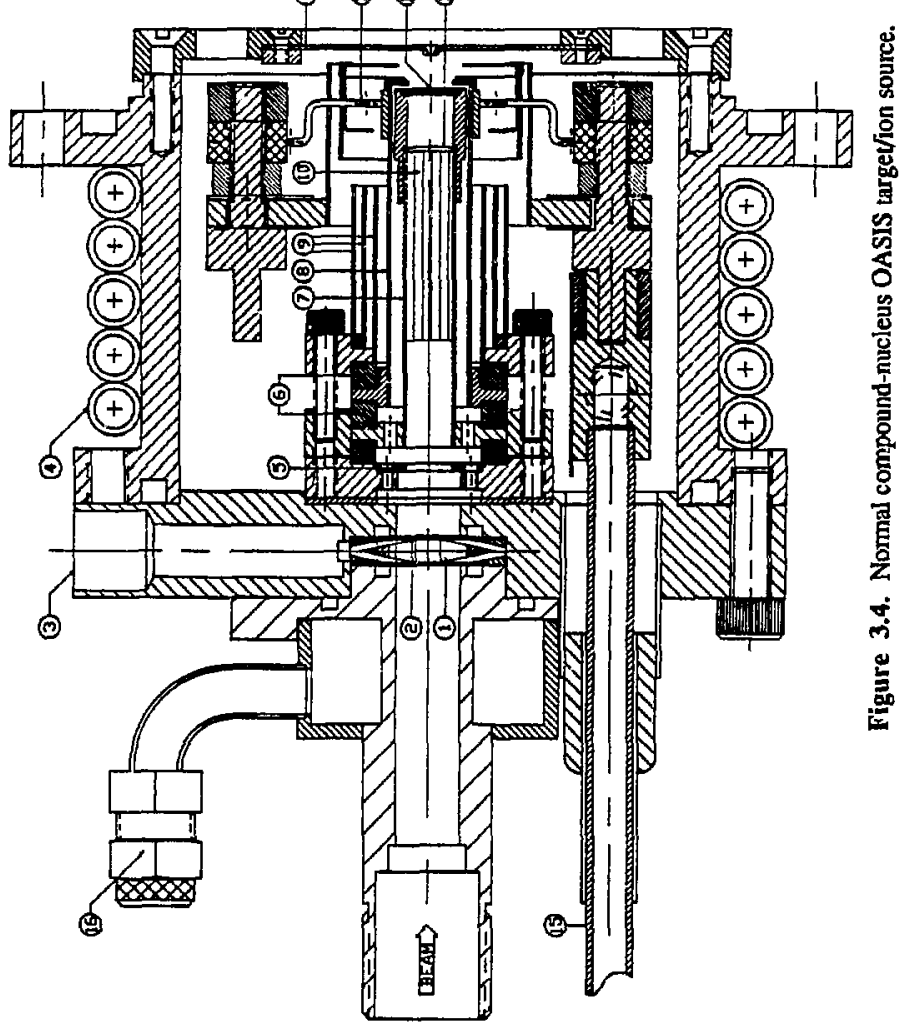


Results of these target/ion source tests will be discussed in chapter 5 . The recoils produced (and caught in the case of the W window/target ion source) in the anode endplate diffused from the catcher and were surface ionized by heating the ionization region of the ion source to temperatures near the melting point of $\mathrm{Ta}(-3000 \mathrm{C})$ by electron bombardment (EB). Most targetion sources lasted $\sim 24$ hours before most of the Ta cylinder wall of the anode had been evaporated by the EB, except for the W window/target source where the $W$ window was melted through by the SuperHIILAC beam in about six hours. Any of these problems caused a noticeable drop in the ion source output at which time a new ion source would be installed.

\section{2b. THE SEPARATOR}

After being ionized, recoils in the ion source would be extracted axially and accelerated to a $50 \mathrm{keV}$ potential. The ion beam was then focused with an einzel lens and a quadrupole triplet onto the entrance slit of the separator magnet. This magnet had the following basic parameters: sector angle $\phi=180^{\circ}$, mean radius of curvature $r_{0}=0.66 \mathrm{~m}$, first field index $\alpha=0.5$, and a mass resolving power $R_{m}=880$ (for 1.5 mm object slit). With the $50-k V$ acceleration, masses from 45 to 380 amu were separable with a transmission of $-95 \%$. The magnetic field was measured with an NMR probe locateci at the $135^{\circ}$ position inside a small homogeneous magnetic field section. $A^{-a l l}$ probe was located next to the NMR probe to tune the NMR probe as the magneac field was changed. The ion energy was accurately determined by measuring the accelerating potential with a 6-1/2 digit digital voltmeter (DVM). A PDP 11/10 minicomputer was used for control of the accrlerating voltage. Using the NMR probe and DVM reading, the computer calculated the mass that would come through the magnet and then applied a correction voltage ( -8 volts to +8 volts) to the 
accelerating potential to separate the desired mass with an accuracy of $\pm 0.01 \mathrm{u}$. Mass calibrations were accomplished after each ion source change by introducing a small amount of a suitable rare earth oxide into the ion sources. This allowed a stable mass marker either at the desired mass or a few mass units away to be produced. The PDP 11/10 minicomputer also monitored and controlled all other important instrumental parameters of the separator.

\section{2c. TRANSPORT SYSTEMS}

After the recoil products had been mass analyzed, the ion beam was deflected $90^{\circ}$ verically to the counting room four meters above the cave. The deflection was performed via an electrostatic mirror operated at $-80 \%$ of the accelerating potential. The transfer line from the electrostatic mirror to the counting room contained two quadrupole triplets and two Faraday cups at the midpoint and counting room end for nuning the beam optics. The counting room was shielded from the radiation produced in the cave by the cave ceiling ( $\sim 18$ inches of concrete), large quantities of polyethylene for neutron thermalization, the counting room floor ( -6 inches of concrete), and $\sim 4$ inches of lead on the counting room floor.

After transport to the counting room, the recoils were collected on a fast cycling tape system. This systern consisted of an IBM 729 tape drive modified to bring a tape loop through an evacuated chamber where the recoils were collected and counted. To bring the tape from the atmosphere into a vacuum of $\sim 10^{-6}$ torr, it was guided through three differentially-pumped feed-through chambers. The activities were then implanted in the magnetic tape directly above the transfer line. After the apjs : priate collection time, the tape was moved 6.88 inches to bring the activity into the detector array. The tape, after leaving the detector chamber, went through a 
second set of vacuum feed-through chambers, identical to the first, and was then ;ound up by the IBM 729 Jrive. Magnetic computer tape with a conductivity of 3-4 $k \Omega$ per square (Scotch 700) was used to prevent electrostatic build-up from the 50 $\mathrm{keV}$ ion beam. For the ${ }^{176} \mathrm{Yb}$ beam experiment, a tape travel time of $\sim 70$ msec was used to move the activity from the collection point to the detector array. For the 170Er beam experiments, this travel time was increased to $\sim 200 \mathrm{msec}$, by using a different gear ratio in the IBM 729 drive to assure better activity positioning in the detector array.

The tape drive was controlled by an Intel 8085 microprocessor. The system was run in a stepping mode where by the activity was collected for a set time and then moved to the detectors to be counted while the next sample was being collected. The collection/counting time intervals were selected based on the known or predicted half-lives [Kla84, Tak83] of the desired activities. The tape control computer inhibited counting during the tape movement plus $-10 \mathrm{msec}$ settling time and also reset and stared a digital timer used for time-tagging of each decay event during the counting interval for half-life determinations.

\section{2d. THE DETECTOR SYSTEM}

Beta decay of :aeutron-rich rare-earths produces measurable electrons, $x$ rays, $\gamma$ rays, and conversion electrons. $X$-ray and $\gamma$-ray singles data were of the most importance in identifying new isotopes. However, $\beta \gamma, \gamma \gamma$, and $x \gamma$ coincidence information and $\beta$ - endpoint energy determinations were essential for synthesizin ; decay schemes. The normal detector set-up used at OASIS for neutron-deficient studies, shown in Figure 3.5, was used for these studies also. The only element of this array not required for this research was the $10.4 \mu \mathrm{m} \mathrm{Si} \mathrm{detector} \mathrm{normally} \mathrm{used} \mathrm{as}$ 


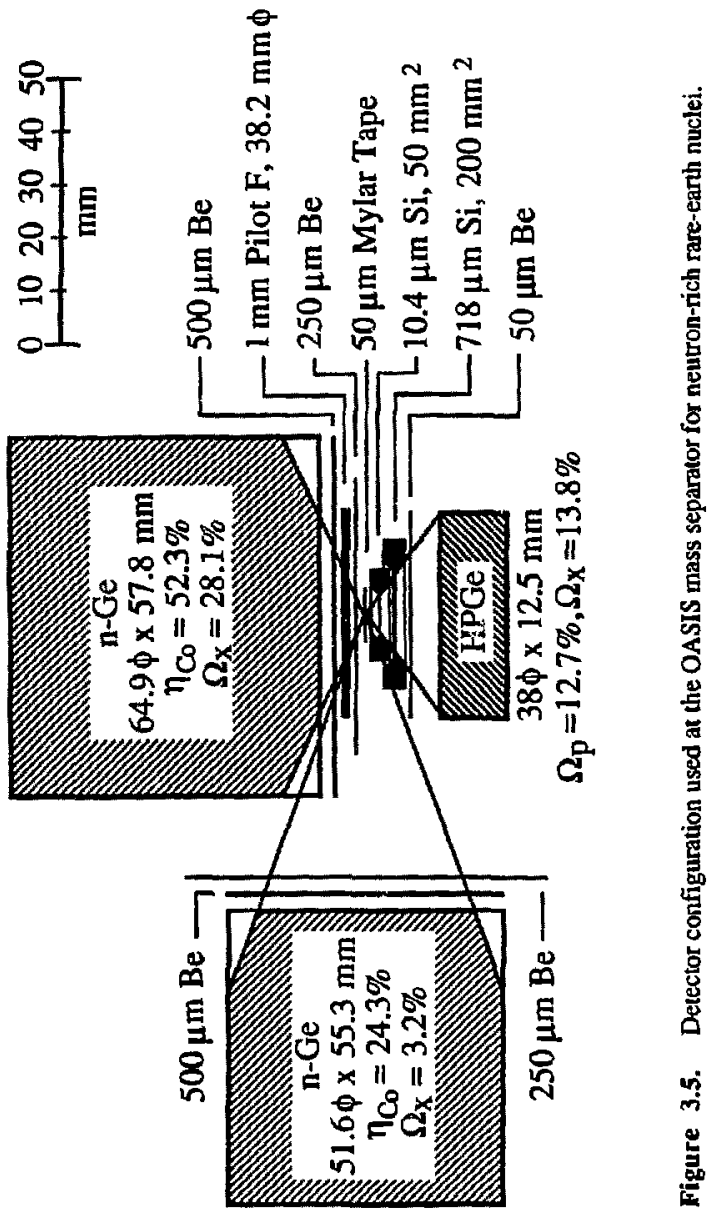


a $\triangle E$ particle detector of a particle telescope. It was not removed due to its fragile nature. Its effect on $\beta$ - energies is to absorb $\sim 3.6 \mathrm{keV}$ for a $1 \mathrm{MeV}$ electron and $\sim 4.1$ $\mathrm{keV}$ for a $5 \mathrm{MeV}$ electron [Pag72], a negligible effect in these experiments.

A beta telescope and low-energy (10-400 keV) $\psi$-ray detector, consisting of a $718 \mu \mathrm{m}$ Si detector and a planar hyperpure Ge (HPGe) detector, faced the radioactive layer on the tape. The $718 \mu \mathrm{m} \mathrm{Si}$ detector was capable of stopping electrons of 0.40 to $0.55 \mathrm{MeV}$ [Pag72] depending on the direction of the electron thru the Si. The remaining energy of a high energy electron would be detected in the HPGe. A large solid angle ( $35 \%)$ 1-mm thick plastic scintillator, for electron and positron detection, and a large ( $52 \%$ relative efficiency) Ge detector, for $\gamma$-ray detection were located on the opposite side of the tape. A third Ge detector (24\% relative efficiency) was located $-4.5 \mathrm{~cm}$ from the source at $90^{\circ}$ to the other detectors. All five detectors used were surrounded by at least 2 inches of lead to shield against background radiation and activities on the tape being collected for the next counting cycle or from previous counting cycles.

Energy calibrations were performed before and after each experiment. In the case of the two-week long ${ }^{170} \mathrm{Er}$ beam experiment, a mid-run calibration was performed. Calibrations of the germanium detectors were performed using standand $\gamma$-ray sources $\left({ }^{152} \mathrm{Eu},{ }^{241} \mathrm{Am},{ }^{226} \mathrm{Ra}\right.$ ). The silicon detector was calibrated with a precision pulser which was periodically calibrated using standard $\alpha$ sources. The long term stability of the pulser was very good. Special thin sources attached to the computer tape and moved to the actual counting position were used to determine the absolute efficiencies of the Ge detectors about once per year. Calibration of the plastic scintillator was not necessary since output from this detector was used only 
for coincidence information to determine $\beta \gamma$ and $\beta x$ coincidences with much higher $\beta$ efficiency than with the $\beta$ telescope.

\subsection{DATA ACQUISITION}

Data acquisition for OASIS consisted of the electronics, computers , and various software discussed in the following sections.

\section{3a. DETECTOR ELECTRONICS}

The pulsed optical preamplifier signal from the HPGe detector was electronically split into a low gain amplifier, for $\beta$ particle detection (0-5 MeV range), and a high gain amplifier, for low energy photons $(0-400 \mathrm{keV}$ range). The low gain signal and the amplified signal of the $718 \mu \mathrm{m} \mathrm{Si} \mathrm{made} \mathrm{up} \mathrm{the} \beta$ telescope signals. The $52 \%$ Ge preamplifier signal was also split into a low gain $(0-4 \mathrm{MeV}$ range) amplifier and a high gain (0-400 keV range) amplifier. Single amplifiers were used for the $718 \mu \mathrm{m} \mathrm{Si}$, plastic scintillator, and $24 \%$ Ge detectors.

Singles data were acquired for the high gain HPGe, low gain $52 \%$ Ge. and the $24 \%$ Ge detectors for determining intensity information of $\gamma$ and $x$ rays. Multiparameter event-by-event data were acquired for $\beta \gamma$, $x \gamma$, and $\gamma$ coincidences. The fast timing signals from each detector, after going through appropriate shaping amplifiers and constant fraction discriminators, were used to define coincident events of interest. Timing information between detector pairs was also generated from the fast timing signals using time to amplitude convertors (TAC's). In the ${ }^{176} \mathrm{Yb}$ beam experiment, coincidences ( $-2 \mu$ s overlap) between the $718 \mu \mathrm{m}$ Si und HPGe detectors ( $\beta$ telescope), the plastic scinillator and the $52 \%$ Ge detector, and pairs of the three germanium detectors defined a coincidence event. These five coincidence 
signals were logically OR'ed to obtain one master event trigger. In the ${ }^{170} \mathrm{Er}$ beam experiment, the same coincidence criteria as in the ${ }^{176} \mathrm{Yb}$ experiment were used plus additional coincidences between the plastic scintillator and the other two germanium detectors (HPGe and 24\% Ge) were added since these would add By coincidences with a large $\beta$ efficiency. High quality linear electronic components were used for amplification, shaping, and gating of all energy signals, resulting in good resolution and stability. Extreme efforts were made to prevent ground loops in electronics, and these efforts were generally successful.

All coincident energy signals, TAC's, and timing information, after the above mentioned electronic processing, were routed to a single CAMAC crate. In the CAMAC crate, the energy and TAC analog signals were converted to digital information with high resolution LeCroy 3511 or Ortec AD811 ADC's. Timing information was digitized using LeCroy scalers. Analog singles data from the HPGe and 52\% Ge detectors were digitized with LeCroy 3512 Buffered ADC's, timerouted ( 8 equal time bins per counting cycle) with LeCroy 3587 Routers, and histogrammed in CES HM2161 64K histogramming memories ( 8 by 8192 channel spectra).

\section{3b. COMPUTERS AND DATA ACQUISITION SOFTWARE}

The main data acquisition computer was a ModComp Classic computer. Interfacing between the computer and various $\mathrm{ADC}$ 's, scalers, and memories was achieved with a CAMAC branch controller and a differential branch driver and transceiver. The CDAS software package [Bel86] was used to read the various event parameters, store the data on magnetic tape, and also supply some on-line sorting and display. The data acquisition architecture allowed a data rate of $>30,000$ parameters 
per second However, the data rate never approached that level in this study. The histogramming memories (HPGe and $52 \%$ Ge singles data) could be read either into the on-line graphics display memory or directly to magnetic tape, with a task nunning at a lower priority than the CDAS software on the ModComp Classic computer. A Tracor Northem 1750 multichannel analyzer was used for pulse-height analysis of the singles data from the $24 \% \mathrm{Ge}$ detector. An Apple Macintosh Plus was used to read out the data from the Tracor Northem and store it on floppy disk.

\subsection{EXPERIMENT SPECIFICS}

This research study consisted of two major experiments: a six-day experiment of ${ }^{176} \mathrm{Yb}$ projectiles which was run in the first week of November 1987, and a twoweek ran (longest continuous run for OASIS to date) of $170 \mathrm{Er}$ ions that was performed in the first two weeks of August 1989. The details for these two runs will be discussed in the following sections. A five-day run was attempied in December 1988, but due to technical difficulties with the SuperHILAC, no ${ }^{170} \mathrm{Er}$ beam was delivered to OASIS.

\section{4a. 176Yb EXPERIMENT}

The SuperHILAC delivered 25-45 particle nancampere beams of $8.40 \mathrm{MeV} / \mathrm{u}$ $176 \mathrm{Yb}$ ions on $\mathrm{W}$ targets using nanural ytterbium in the ion sources $(176 \mathrm{Yb}$ abundance $=12.7 \%$ ). The purpose of this experiment was to produce new isotopes at masses $A=171$ and 174. The OASIS mass separator was mass calibrated using $\mathrm{Yb}_{2} \mathrm{O}_{3}$, which pmvided mass markers at $\mathrm{A}=171$ and 174. Most of the targetion sources used in OASIS were of the nomal geometry (capillaries, W endplate, no windows). One special ion sounce was used which had capillaries, a Ta endplate, 
and a $17 \mathrm{mg} / \mathrm{cm}^{2} \mathrm{~W}$ window/target at the end of the capillaries (position 14 in Figure 3.4). The event-by-event data consisted of 15 parameters (event tag, clock parameter, time (from last tape movement), 7 energy parameters and 5 TAC's) per event.

\section{4b. ${ }^{170}$ Er EXPERIMENT}

The SuperiIlLAC provided 50-125 pnA of $8.5 \mathrm{MeV} / \mathrm{s} 170 \mathrm{Er}$ ions on W targets using natural erbium ion sources $\left({ }^{170} \mathrm{Er}\right.$ abundance $\left.=14.9 \%\right)$ and ion sources with ${ }^{170} \mathrm{Er}$ enriched strips spot-welded to natural erbium backings. The purpose of this experiment was to produce new isotopes at mass $A_{A}=169$ and construct decay schemes of ${ }^{168} \mathrm{Dy}$ and ${ }^{171} \mathrm{Ho}$. The OASIS mass separator was mass calibrated using $\mathrm{Yb}_{2} \mathrm{O}_{3}$ for masses $\mathrm{A}=168$ and 171 . Mass $\mathrm{A}=169$ was calibrated by interpolating between the $A=168$ aral 170 mass calibrations of the $\mathrm{Yb}_{2} \mathrm{O}_{3}$. Two special arget/ion source geometries were run in DASIS besides the normal geometry (capillaries, W endplate, no window): one geomery with capillaries, $W$ coated Ta endplate, and no window and the other geometry with no capillaries, Ta endplate, and a $12 \mathrm{mg} / \mathrm{cm}^{2}$ target/window at position 14 in Figure 3.4. The event-by-event data consisted of 17 parameters (event tag, clock parameter, time (from the last tape movement), beam parameter (for ion source test normalization), 7 energy parameters, and 6 TAC's) per event. 


\section{DATA ANALYSIS}

The data acquired in the experiments performed cas 'e grouped into three subsets: the gamma singles data, the event-by-event coincidence information, and the beta telescope spectra.

\subsection{GAMMA SINGLES DATA}

The singles data (ModComp tapes or Macintosh floppy disks) were first transferred to Digital Electronic Corporation (DEC) computers. In the case of the Macintosh data, commercial Kermit file transfer programs were used. The ModComp tapes were read with software developed in the OASIS group. The two DEC computers used in the analysis of the singles data were a VAX 8650 Cluster at LBL or a $\mu$ VAX-II Workstation owned by the OASIS group. The 8650's cpu and YO were almost an order of magnitude faster for the analysis programs used than the HVAX, however, dae to the 8650 being a time-sharing machine, their speed increase was not realized under actual conditions. All spectra were converted to an identical binary format to minimize disk memory usage.

The main program used to analyze the singles spectra was a VAX version of SAMPO [Rou69]. This version allowed for the fitting of up to eight $\gamma$ rays, as compared to only six in the original code, in a single fitting region. This new option was very useful in fitting of $K_{\alpha}$ and $K_{\beta} x$-ray regions, where four adjacent elements were detected. The shape parameters, the key variables in SAMPO to assure good fits, were generated from the calibration specis of each experiment. Separate shape parameters were used for $y$ rays and $x$ rays due to slightly different shapes of their 
peaks. Efficiencies used were from the once per year calibrations and are shown in Figure 4.1.

Intensity versus time information, from the $52 \% \mathrm{Ge}$ and HPGe detectors, was fit with a least-squares error-weighted decay program [Gre85]. This program allowed either up to five independent components, or growth and decay with up to four independent components, or two growth and decay components with up to one independent component. However, since the hardware used created only eight timebinned histograms for these detectors, no more than three half-lives were fit at one time. The program would calculate a $\chi^{2}$ goodness of fit parameter and a reduced $\chi^{2}$, $\chi^{2} /$ (\# of points - \# of free parameters - 1). Both of these parameters will be shown with the decay curves presented in chapter 5 .

For the determination of absolute $\gamma$-ray intensities, information from the $52 \%$ Ge had to be treated with caution. Due to the large size of the crystal and the proximity of the source, summing problems were noted. This was not a count rate problem ("pile-up"), but a true gamma cascade summing problem. The errors this introduced were at the largest when one of the $\gamma$ rays in a cascade was at at an energy near the peak of the $52 \%$ Ge detectors efficiency ( $150 \mathrm{keV}$ in Figure 4.1). Summing problems were not seen in the HPGe detector, and the $24 \%$ Ge detector was far enough from the source to avoid this problem. Gamma intensities reported in this study are from the HPGe and $24 \%$ Ge detectors, unless noted. In a few cases, the order of magnitude difference in the efficiencies of the the two $\mathrm{Ce}$ detectors (Figure 4.1), would cause the desired gamma ray to be undetectable in the $24 \% \mathrm{Ge}$ detector above the various backgrounds and the intensities from the $52 \% \mathrm{Ge}$ were used. 


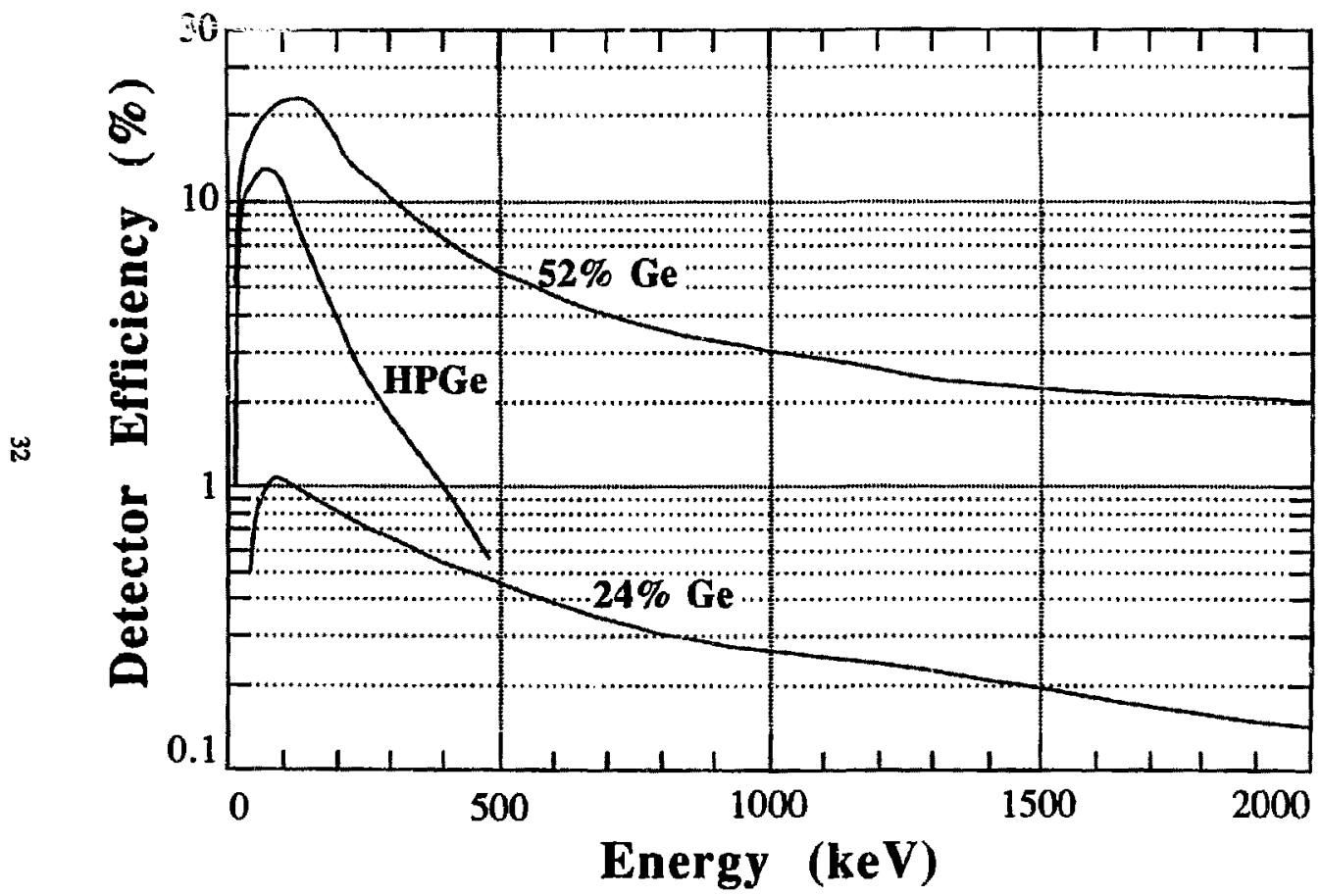

Figure 4.1. Absolute efficiencies from 15 to $2000 \mathrm{keV}$ for the germanium detectors in the geometry used. 


\subsection{CONNCDENCE DATA}

The event-by-event data were aralyzed with a ModComp Classic computer. The EVA software package [Bel87a] was used exclusively to produce the various histograms. Appropriate peak and background gates would be set in a chosen detector and coincidence spectra from the other detectors would be generated. Beta telescope spectra were produced based on the following requirements: energy signals above $0.18 \mathrm{MeV}$ in the $718 \mu \mathrm{m} \mathrm{Si}$ detector and $0.15 \mathrm{MeV}$ in the HPGe low gain signal plus a very narrow gate on the prompt TAC peak of the $718 \mu \mathrm{m}$ Si/HPGe TAC. These gates were used to avoid noise in both detectors and to obtain clean signals with the narrow TAC. Other coincidence spectra were normally produced with "wide-open" TAC gates. After $\gamma, x \gamma$, or By coincidences were found, TAC spectra were generated after gating on both signals of the coincidence pair to determine if the intermediate level in the decay was delayed.

The peaks in the histograms generated with EVA were fit with the SUSIE graphics software package [Bel87b]. The program gave graphic displays of the histograms plus arithmetic functions to operate on the spectra. Background-gated spectra could be subtracted from peak-gated spectra and stored on disk or tape. Simple peak-fitting calculations could also be performed and hardcopy plots could be quickly generated.

After use of the one-dimensional features of the software mentioned above, two-dimensional feanures were noticed and used. Two-dimensional energy spectra were generated for any pair of detector signals (the beta telescope is only one signal in this discussion) with EVA. The SUSIE software package allowed for quick interactive background-subtracted gates to be set in one direction of the 2-D spectrum 
and produce the resulting 1-D projection. This procedure allowed for quicker results once the 2-D spectra were generated. Generation of the 2-D spectra was about an order of magnitude slower than generating 1-D spectra due to the size difference in the spectra. The flexibility of the 2-D histogram more than made up for this speed difference, since all possible gates in either detector could be tested without having to resort the event-by-event data.

Spectra generated with either software package could be read into the DEC computers and fit with SAMPO, in the case of $\gamma$ spectra, or the $\beta$ analysis software (to be described below), in the case of $\beta$ spectra.

\subsection{BETA TELESCOPE SPECTRA}

The spectra measured with the beta telescope in this study proved to contain extremely important information. Unlike the neutron-deficient studies normally done at OASIS, where $\mathrm{K} \times$ rays were seen in a large fraction of the decays due to the large electron-capture branches, neutron-rich decays only produce $x$ rays if a $\gamma$ transition in the decay is internally converted. Thus, the elemental assignment from the $\mathrm{K} x$ rays was almost absent as a means to identify a specific isobar in the mass chain separated. However, elecurons from $\beta$ - decay could be detected with good resolution in the beta telescope. The electrons of a paricular decay become hard to distinguish from beta particles of other decays due to the energy of $\beta$ - decay being randomly split between the electron and the electron antineutrino. However, in odd mass chains, the total beta decay energies, $Q_{\beta}$, generally increase with the neutron number in an isobaric chain. This fact coupled with possible ground state or low excitation energy level beta feedings yields for the lowest $Z$ isobar a portion of the beta spectrum beyond the beta endpoints of isobars closer to the line of beta stability. The increase 
in $Q_{\beta}$ values for more neutron-rich nuclei is not always seen in even mass chains due to the odd-even affect of nucleons on masses. The $Q_{\beta}$ value for an even-even isotope is generally less than the $Q_{\beta}$ values of it's two neighboring odd-odd isobars. However, if an odd-odd decay was studied, it's $Q \beta$ value was generally greater than the isobars closer to beta stability.

Being able to determine new beta decays and intensities from only the upper part of a beta spectrum was needed. Integration of regions of the time-binned beta spectra beyond known beta endpoints wolld yield half-life information for the beta decay. Background subtraction was performed by using an identically sized window at the higher energy part of the beta spectrum (background region) normalized with a ratio factor determined for the same windows in a 15-hour beta background spectrum. A computer code was written to calculate Fermi-Kurie plois in order to determine beta endpoints. Examples of this calculation are shown in Figures 4.2 and 4.3 , where endpoints of $\beta^{-}$particles coincident with gamma rays seen in the $52 \% \mathrm{Ge}$ detector from the decay of ${ }^{168} \mathrm{Ho}$ are calculated. An error-weighted least-squares fit was used to determine the best linear fits shown in the figures and the corresponding endpoints.

Determination of absolute beta intensities was not as simple as determining gamma intensities. A large fraction of beta counts were lost in the determination of the beta telescope energy below $\sim 0.35 \mathrm{MeV}$ (due to the 0.18 and $0.15 \mathrm{MeV}$ threshold gates in the $718 \mu \mathrm{m} \mathrm{Si}$ and HPGe low gain signals). Also for ground state beta branches, :zo gamma-ray gating could be used, so only the part of the beta spectrum beyond the known beta endpoints (the very low statistics portion of a beta spectrum) could be used. Using some subroutines from a code to calculate the response of Ge detectors to positrons [A vi81], a computer program was written that would generate 


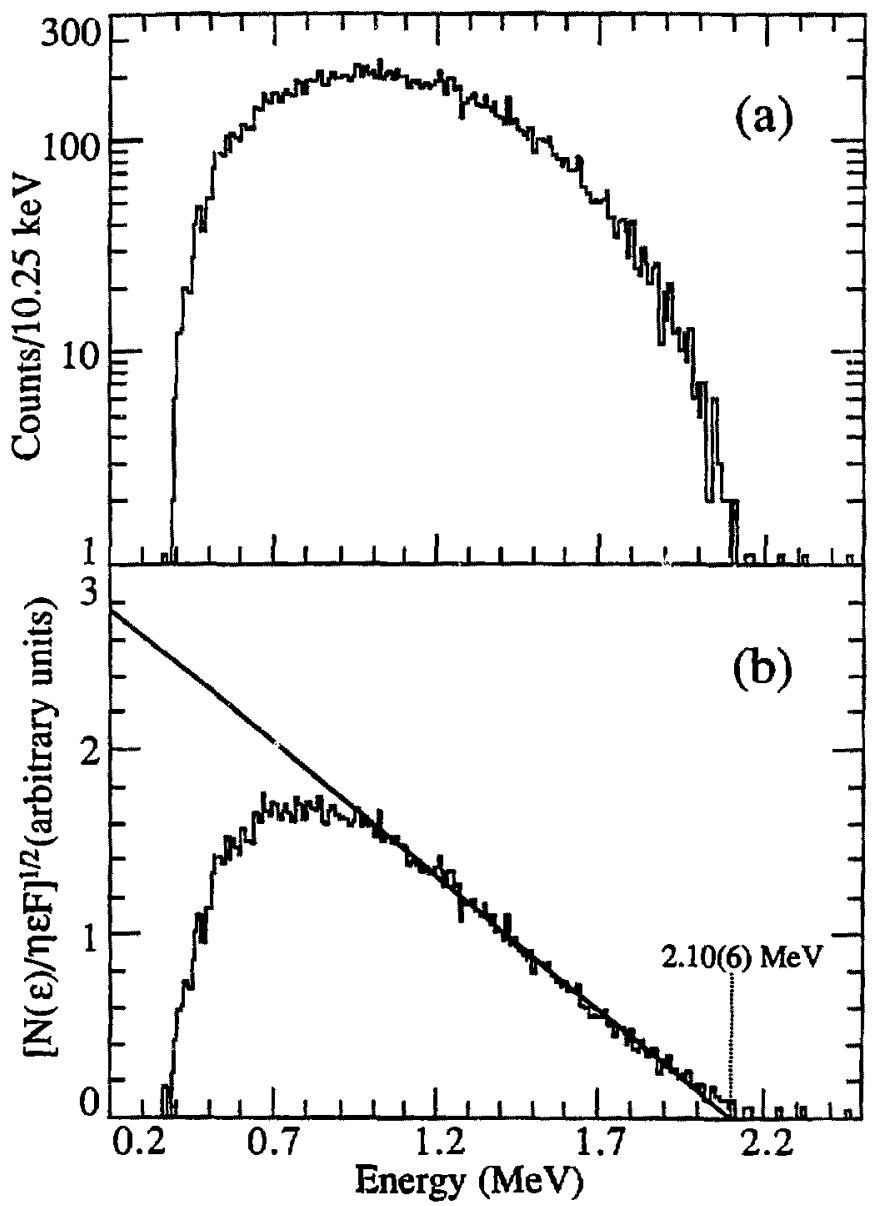

Figure 4.2. Beta decay data coincident with $741-\mathrm{keV}$ gamma rays in the $52 \% \mathrm{Ge}$ detector for the $A=168$ mass chain; (a) beta particle spectrum measured wh the beta telescope and (b) Kurie plot and least-squares lixear fit from 1.1 to $2.0 \mathrm{MeV}$. 


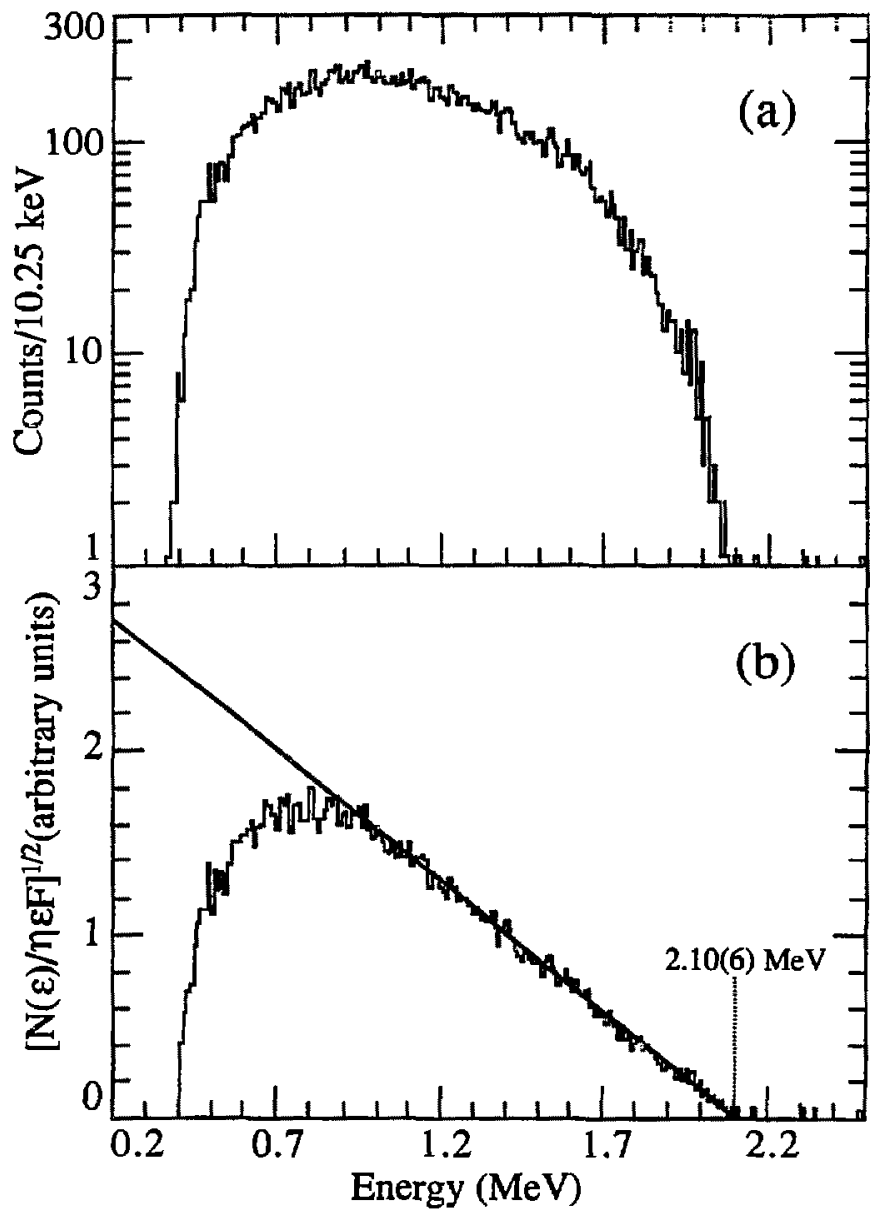

Figure 4.3. Beta decay data coincident with $821-\mathrm{keV}$ gamma rays in the $52 \%$ Ge detector for the $A=168$ mass chain; (a) beta particle spectrum measured wh the beta telescope and (b) Kurie plot and least-squares linear fit from 1.1 to $2.0 \mathrm{MeV}$. 
an ideal allowed $\beta$ - spectrum. It required only the $Z$, A, endpoint energy, and energy calibrations to generate a calculated $\beta$ spectrum. A copy of the source code used to calculate these calculated $\beta$ spectra is given in Appendix A. A calculated spectrum would then be renormalized to the measured spectrum such that the number of the counts in the clean upper region of the measured spectrum equaled the number of counts in the calculated spectrum over the same region. Figure 4.4 shows the fit of calculated spectra to the measured $\beta$ spectra in Figures 4.2 and 4.3. The absolute efficiency of the beta telescope was determined from these calculated spectra. The rotal counts of these two calculated spectra were divided by the number of gamma tays seen in the $52 \%$ Ge singles data, used as gates to generate the measured beta spectra. A $\beta$-detection efficiency of $8.47 \%$ was determined by this methad for the energy and TAC gates used. The dependence of this efficiency on different endpoint energies was not determined. 


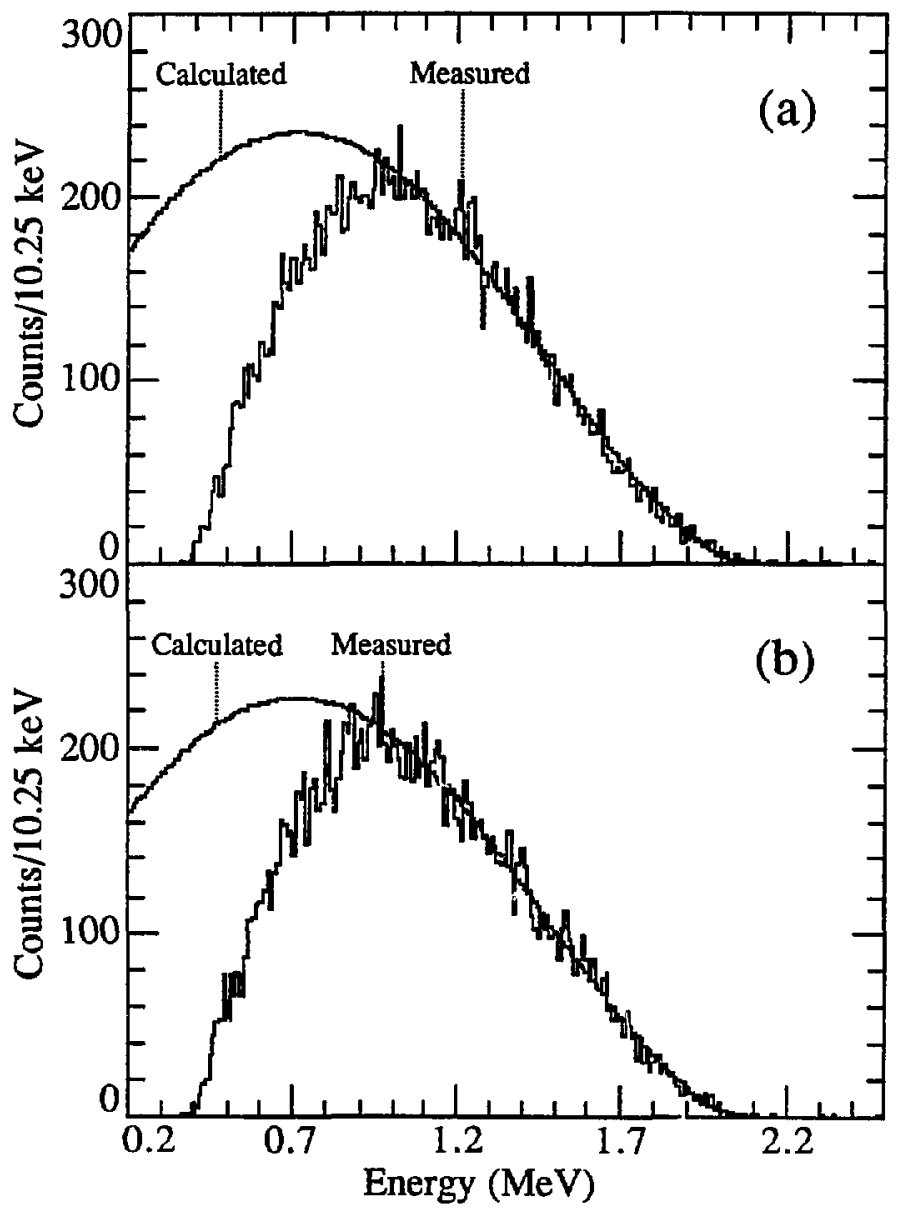

Figure 4.4. Beta decay data and calculated spectra fits in the $A=168$ mass chain coincident with gamma rays in the $52 \%$ Ge detector at; (a) $741 \mathrm{keV}$ and (b) $821 \mathrm{keV}$. 


\section{RESULTS}

In the course of this research, two new isotopes, ${ }^{169} \mathrm{D} y$ and ${ }^{174} \mathrm{Er}$ [Cha89], and the isomer ${ }^{168} \mathrm{Ho}^{\mathrm{m}}$ were discovered. In addition, the decay schemes of two previously identified isotopes, ${ }^{168} \mathrm{Dy}$ [Geh82] and ${ }^{171} \mathrm{Ho}$ [Ryk89], were determined. Figure 5.1 shows location of these isotopes in the chart of the nuclides. These results will be discussed in the following sections in increasing mass number. The $A=168$ isotopes will all be discussed in one section due to the related data analysis. For historical purposes, the chronological onder of the production of these isotopes is as follows: ${ }^{174} \mathrm{Er}-11 / 6 / 87,{ }^{171} \mathrm{Ho}-11 / 8 / 87$ and $8 / 7 / 89, \mathrm{~A}=168$ isotopes $-8 / 2 / 89$, and ${ }^{169} \mathrm{Dy}-8 / 4 / 89$. The results of the various OASIS target/ion source tests are also presented.

A few standard short-hand notations used in decay scheme studies are followed in this presentation. Internal conversion coefficients (ICC) will be denoted by $\alpha_{\mathrm{tat}}, \alpha_{\mathrm{K}}, \alpha_{\mathrm{L}}$, etc. to represent the total, $\mathrm{K}$-sheil, L-shell, etc. conversion coefficients. All ICC's listed in tables are theoretical values [Ros78]. Only $\alpha_{K}$ values could be measured with the equipment. Experimental values followed by numbers in parenthesis have an experimental error equal to the value of the number(s) in parentheses on the last digit(s) of the listed value. Errors are one sigma limits ( $68 \%$ confidence limits). All beta endpoints determined from FermiKurie plots were calculated using a least-squares fiting program with errorweighting. $\beta$ - decays in this region are Gamow-Teller allowed $(\Delta \mathrm{I}=0$ or $1, \Delta \pi=$ no) or first-forbidden ( $\Delta I=0$ or $1, \Delta \pi=$ yes) decays. The relevant selection rules for electromagnetic multipole radiation [Seg77] are reproduced in Table 5.1. The original computer code used by Takahashi et ol. [Tak73] to calculate nuclear half- 


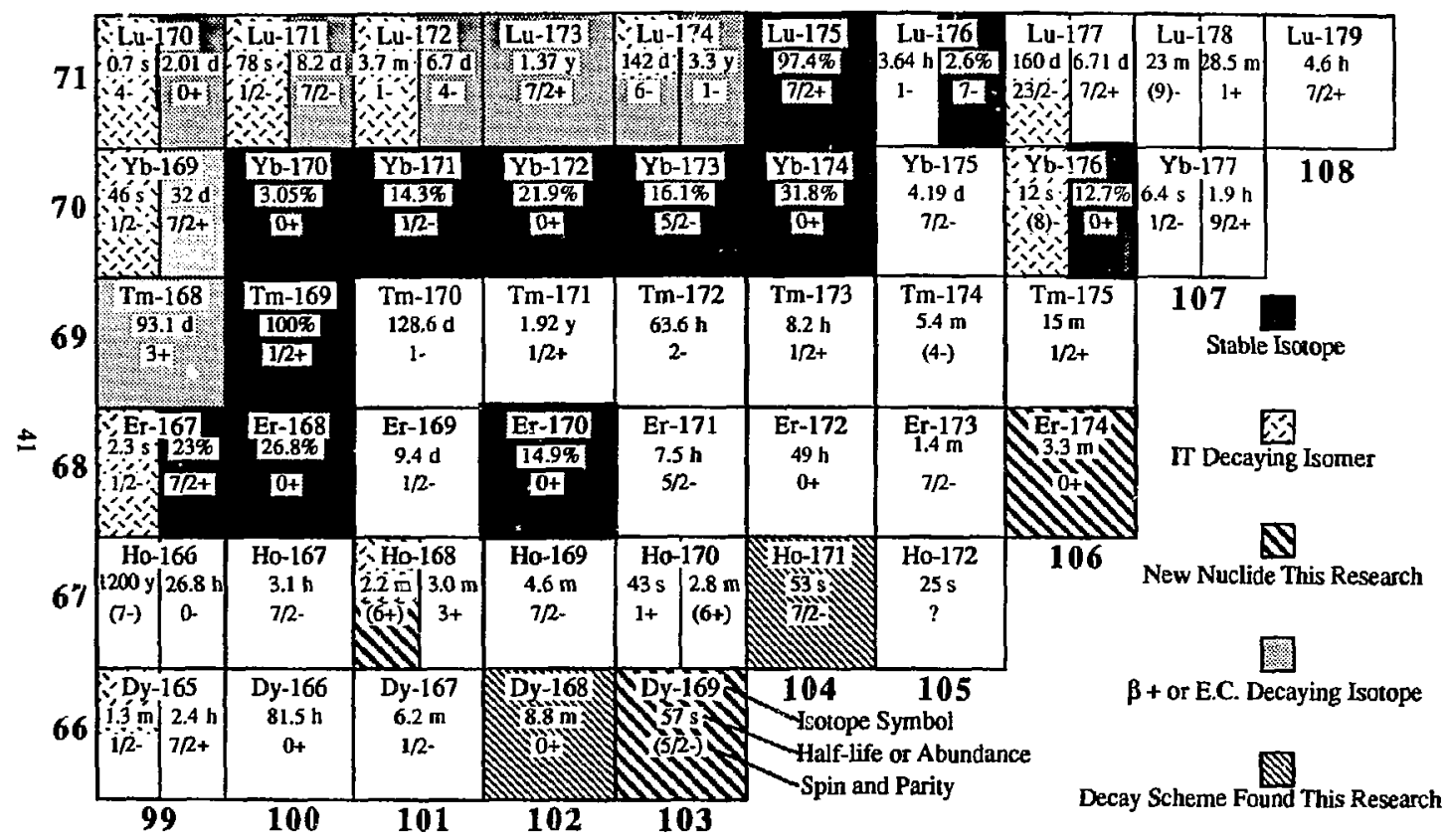

Figure 5.1. Chart of nuclides for the region studied. Isotopes investigated in this research are indicated. All unshaded nuclides decay by beta emission. The two projectile ions used in this research have bold borders. 
Table 5.1. Selection rules for electromagnetic multipole radiation [Seg77] used in this research.

\begin{tabular}{ccccc}
\hline & E1 & E2 & E3 & E4 \\
\hline$\Delta x$ & yes & no & yes & no \\
$\Delta I \leq$ & 1 & 2 & 3 & 4 \\
\hline$\Delta \pi$ & M1 & MR & M3 & M4 \\
\hline$\Delta I \leq$ & no & yes & no & yes \\
\hline
\end{tabular}

lives was obtained from the author [Tak88]. Using $Q$ values calculated with more recent nuclear mass formulae [Hau88] this Gross Theory of Beta Decay (GTBD) code predicted half-lives with better accuracy than before.

\section{$5.1 \quad A=158$ MASS CHAIN}

The $A=168$ mass chain was studied using $170 \mathrm{Er}$ projectiles with various OASIS targetfion source geometries. The discussion of the different sources used and the results will be presented in a later section. Natural erbium was used as the source material for the SuperHIILAC ion sources. Beam intensities of 33 to 75 particle nanoamperes on target werts delivered for about 30 hours. This experiment was performed to determine the decay scheme of the 8.5(5) $\mathrm{m}^{168 \mathrm{Dy}}$ [Geh82] and investigate the affect of various target/ion source geometries on the production of $3.0 \mathrm{~m}^{168} \mathrm{Ho}$ [Wil60]. Thus, a tape cycle time of $1024 \mathrm{~s}(17.1 \mathrm{~m})$ was used to study ${ }^{168} \mathrm{Dy}$, and $512 \mathrm{~s}(8.5 \mathrm{~m})$ was used to study ${ }^{16{ }^{\circ}} \mathrm{Ho}$. During the analysis of the ${ }^{168} \mathrm{Dy}$ data, eviderce of a new isomer ${ }^{168} \mathrm{Ho}$ was seen. Also a new $Q_{\beta}$ - value for ${ }^{168} \mathrm{Ho}$ [Kaw73] was determined. 


\section{1a ${ }^{168}$ Dy DECAY SCHEME}

${ }^{168} \mathrm{C}$ / was identified by researchers at Idaho National Engineering Laboratory from the spontaneous fission of ${ }^{252} \mathrm{Cf}$ and assigned a half-life of $8.5(5)$ minutes [Geh82]. They used a rapid chemical separation system, ESOL Elemental Separation $Q$ Qn-Line) to prepare Ho/Dy fraction samples. Five new gamma rays at $143,192,443,487$ and $630 \mathrm{keV}$ were seen to desay with the average half-life given above and were assigned to the decay of ${ }^{168} \mathrm{Dy}$. They also assigned an emission probability per beta decay of 0.22 (4) for the $487-\mathrm{keV}$ gamma ray from intensity ratios with the $821-\mathrm{keV}$ gamma ray from ${ }^{168} \mathrm{Ho}$ at various decay times.

In the 1024-s tape cycle time data of this research, all five of the gamma rays previously reported were observed to decay with the reported half-life. In addition, two new gamma rays, with energies of 43.8 and $437.0 \mathrm{keV}$, were idenified to decay with the same half-life. A weighted average value of $8.8(3) \mathrm{m}$ was determined for the half-life of these seven gamma rays. In the $x$-ray singles data, Ho $K_{\alpha 1}$ and $K_{\alpha 2} x$ rays were observed. However, these $x$ rays exhibited a twocomponent decay behavior. Figure 5.2 shows the decay curves of the $\mathrm{Ho}_{\alpha 1}$ and $\mathrm{K}_{\mathbf{\alpha} 2} \mathbf{x}$-ray activities. The long half-life had to be held fixed or the fitting program would diverge. The short-lived activity deduced from these curves will be discussed in the section on the ${ }^{168} \mathrm{Ho}^{\mathrm{m}}$ isomer. The intensities of the $8.8 \mathrm{mHo}$ $K_{\alpha 1}$ and $K_{\alpha 2}$ activities were determined by integrating the long-lived conponent of the decay curves.

The absolute decay intensity of ${ }^{168}$ Dy could be determined by observing the growth and decay of its daughter activity, $3.0 \mathrm{~m}^{168} \mathrm{Ho}$. However, this was complicated by the shor-lived activity observed in the $\mathrm{x}$-ray singles analysis. This 


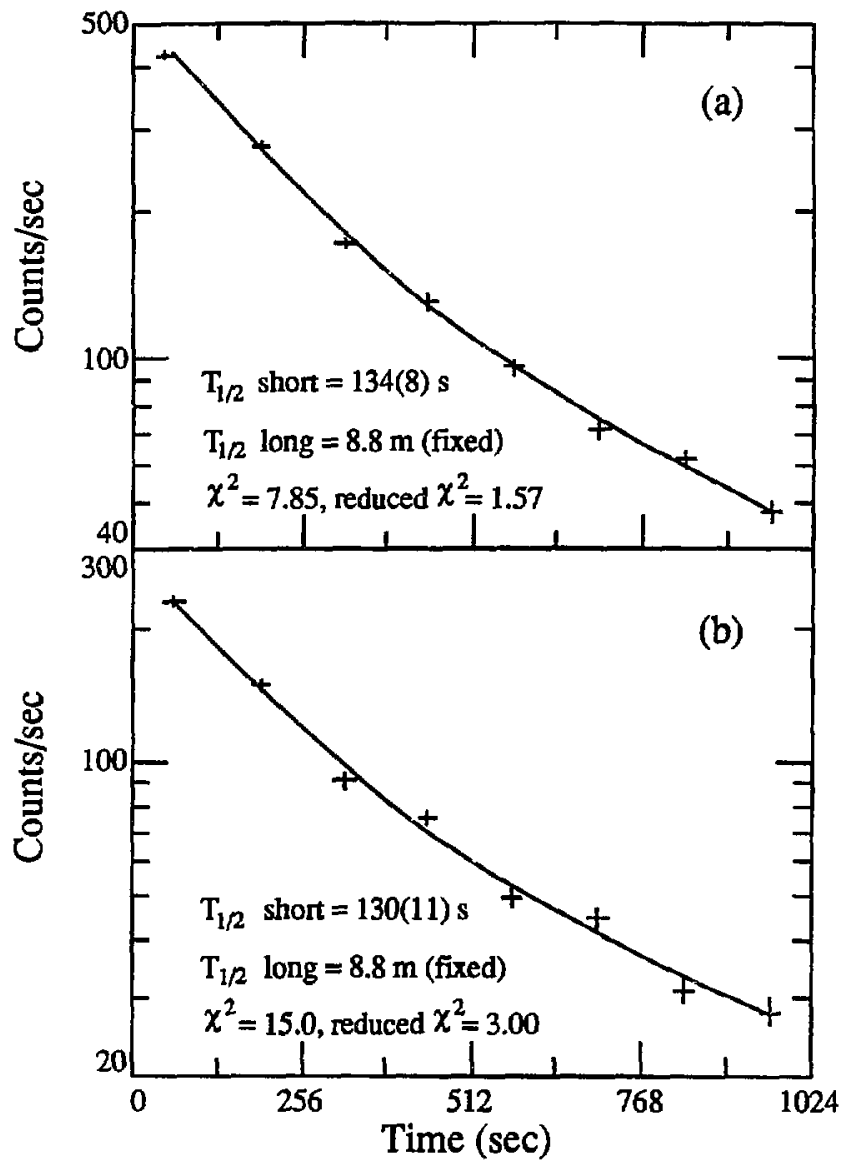

Figure 5.2. Two-component decay curves fit to the Ho x-ray data for the 1024-s rin; (a) Ho $K_{\alpha 1}(47.5 \mathrm{keV})$, and (b) Ho $\mathrm{K}_{\alpha 2}(46.6 \mathrm{keV})$. Vertical bars are indicative of the error in the activity at each point. Horizontal bars are for plotting purposes only and have no relationship to the uncerainty in time (which was assumed to be negligible). 
short-lived activity also decays to ${ }^{168} \mathrm{Ho}$ and aids another growth component to the gamma rays of ${ }^{168} \mathrm{Ho}$. Figure 5.3 shows the decay curves of the two strongest gamma rays (741 and $821 \mathrm{keV}$ ) in the decay of ${ }^{168} \mathrm{Ho}$. Double growth and single decay fits of the ground ( $\left.{ }^{168} \mathrm{Dy}\right)$ and isomeric $\left({ }^{168} \mathrm{Ho}^{\mathrm{m}}\right)$ parent decays feeding the single daughter activity ( $\left.{ }^{168} \mathrm{Ho}\right)$, with all half-lives held fixed, are shown in the figures. (The choice of $132 \mathrm{~s}$ for the short-lived parent will be justified in the next section.) This fit was performed using two growth and decay components with one of the daughter's initial activity held fixed at zero to help convergence. The decay fit in Figure 5.3 may look like only one-component decay, but the fit shown gave a better $\chi^{2}$ value than single- or double-component decay or one growth and decay component. The ratio of the initial intensities for the short- to long-lived parent activities was $\sim 7.2$ for the fit in Figure 5.3 during the 1024-s run. The intensity of the $8.8-\mathrm{m}$ parent, ${ }^{168} \mathrm{Dy}$, was integrated over the counting interval and corrected for the known branching ratios of the 741 - and $821-k e V ~ \gamma$ rays in 168 Ho decay, 35.9(8)\% and 34.7(8)\%, respectively [Led78]. An emission probability per beta decay of 0.225 (16) was determined for the $487.0-\mathrm{keV}$ gamma ray in ${ }^{168}$ Dy decay in good agreement with the reported value 0.22(4) [Geh82].

Coincidence gates were set for the seven $\gamma$ rays and the $\mathrm{Ho}_{0} \mathrm{~K}_{02} \times$ ray in the HPGe or 52\% Ge detectors and the corresponding spectra were generated for coincidences in the other $G$ detector without the gate, the $\beta$ telescope, or the plastic scintillator. A "clean" gate for the Ho $K_{\alpha 1} \times$ ray could not be set due to interterence from $\mathrm{Er} \mathrm{K}_{\omega_{2}} \times$ rays $(48.2 \mathrm{keV})$ of the ${ }^{168} \mathrm{Ho}$ decay. Table 5.2 lists the seven gamma- and Ho $K_{\alpha} x$-ray energies, absolute intensities, multipolarities, conversion coefficients ( $\alpha$ 's), and gamma-ray coincidences assigned to the decay of $8.8 \mathrm{~m}$ 168 Dy. The proposed partial decay scheme, put together from the coincidence and 


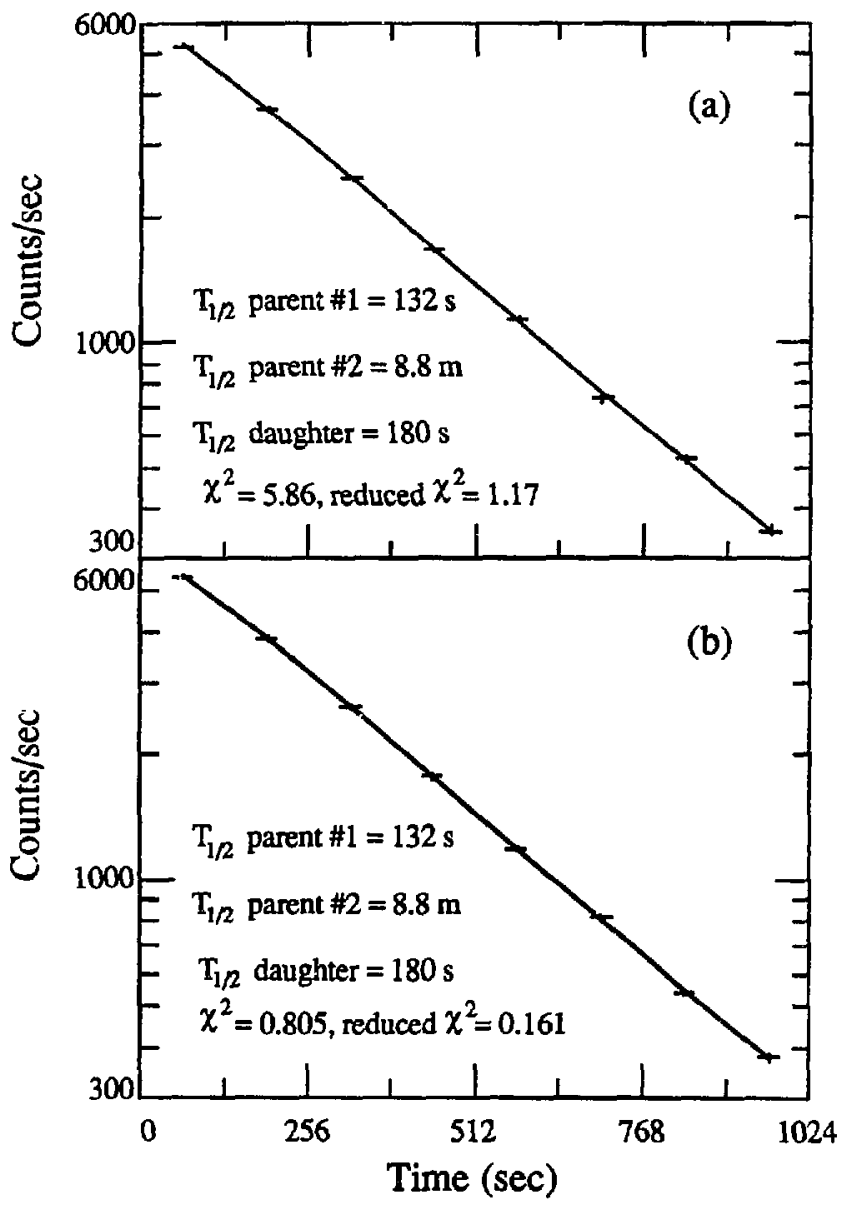

Figure 5.3. Calculated double growth and decay curves of ground and isomeric parent decay feeding one daughter activity, $3.0-\mathrm{m} \mathrm{Ho}-168$, for the 1024-s run; (a) 741-keV gamma-ray decay and (b) 821 -keV gamma-ray decay. All half-lives were held fixed. 
Table 5.2. Gamma-ray energies, $E_{\gamma}$, absolute intensities, $I_{\gamma}$, multipolarities, $M$, theoretical conversion coefficients [Rös78], $\alpha_{\mathrm{K}}$ and $\alpha_{\mathrm{LOt}}$ and gamma coincidences in the decay of ${ }^{168} \mathrm{Dy}$.

\begin{tabular}{|c|c|c|c|c|c|}
\hline$E_{Y}(\mathrm{keV})$ & $I_{Y}$ (abs.) & $\mathbf{M}$ & $\underline{\alpha \underline{K}}$ & $C_{\text {iot }}$ & Coincident $\gamma$ rays ${ }^{b}$ \\
\hline $43.8(2)$ & $0.044(4)$ & (M1) & 0 & 4.90 & $X,(143), 443$ \\
\hline $46.6 \mathrm{Ho} \mathrm{K}_{\alpha 2}$ & $0.122(14)$ & & & & $43.8, X,(143), 443,487$ \\
\hline $47.5 \mathrm{Ho} \mathrm{K}_{\alpha 1}$ & $0.213(23)$ & & & & c \\
\hline $143.5(2)$ & $0.065(5)$ & (M2) & 5.12 & 6.72 & $43.8,487$ \\
\hline $192.5(2)$ & $0.328(20)$ & E2 & 0.180 & 0.279 & $(\mathrm{X}), 437$ \\
\hline $437.0(7)$ & $0.085(11)$ & (M1) & 0.0398 & 0.0465 & $x, 192$ \\
\hline $443.3(2)$ & $0.155(11)$ & (E1) & 0.00600 & 0.00703 & $43.8, \mathrm{X},(143)$ \\
\hline $487.0(2)$ & $0.225(16)$ & (E1) & 0.00486 & 0.00569 & $X,(143)$ \\
\hline $630.4(3)$ & $0.136(11)$ & (E2) & 0.00730 & 0.00897 & d \\
\hline
\end{tabular}

a () indicates the multipolarity was not measured but inferrod from the decay scheme.

b $\mathrm{X}=\mathrm{Ho} \mathrm{K} X$ rays. ( ) indicates a weak coincidence.

c Due to $\mathrm{Er}_{\mathrm{K}} \mathrm{K}_{\infty 2}$ interferences, no clean coincidence gate could be set.

d No coincidences were measured.

intensity information, is shown in Figure 5.4. The gamma intensities listed in Table 5.2 were obtained from the $52 \%$ Ge detector due to the masking of the ${ }^{168} \mathrm{Dy}$ gammas by ${ }^{168} \mathrm{Ho}$ activity in the $24 \%$ Ge detector. The intensity of the ${ }^{168} \mathrm{Ho}$ activity was orders of magnitude greater than that of ${ }^{168} \mathrm{Dy}$ in these experiments. All the gamma rays assigned to ${ }^{168}$ Dy decay and the $\mathrm{Ho} \mathrm{K}_{\alpha} \times$ rays were observed in coincidence with $\beta$ - particles in the beta telescope and the plastic scintillatm. Due to 168 Ho interferences, $\beta$ - energy endpoint analysis could not be performed for ${ }^{168} \mathrm{Dy}$ decay. An average value of $1.4 \mathrm{MeV}$ was obtained from the mass formulae 


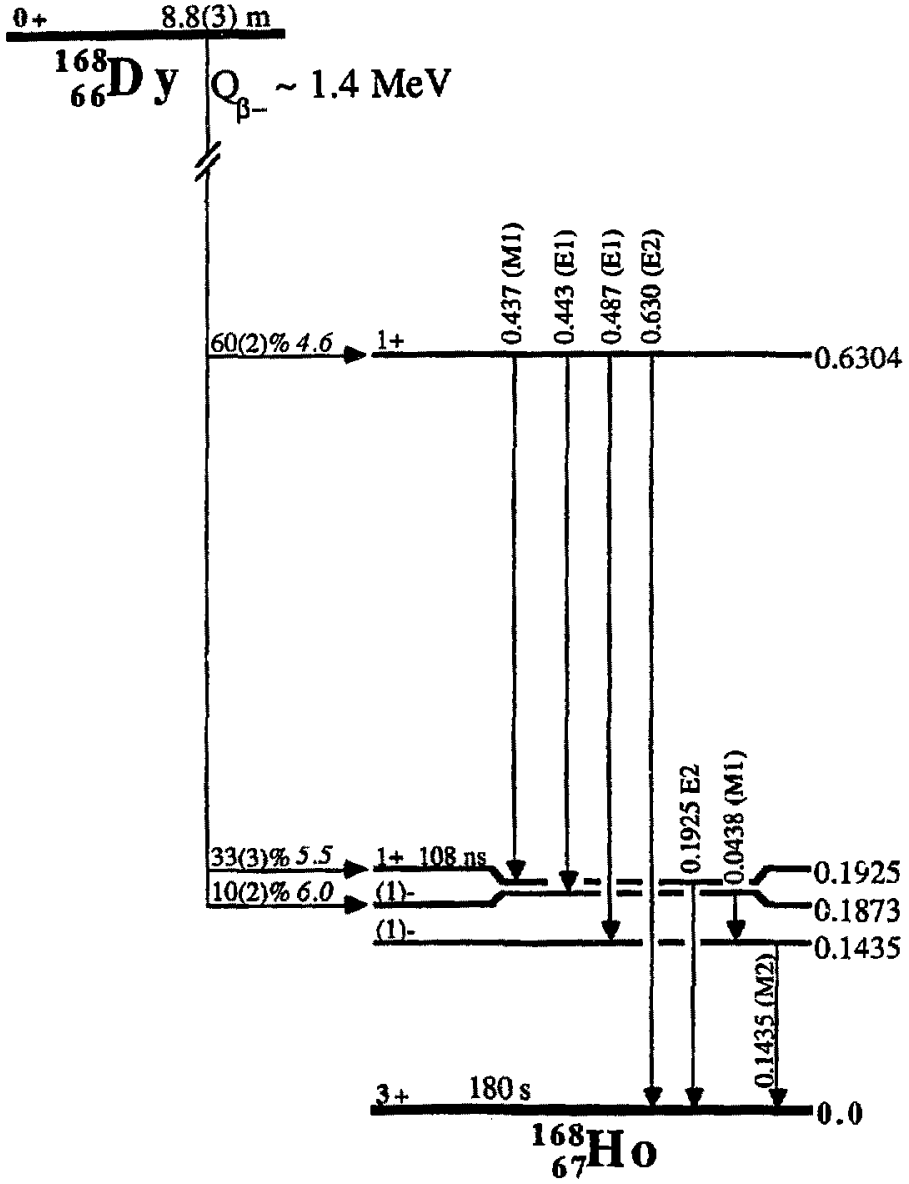

Figure 5.4. Proposed decay scheme for the decay of 8.8(3) $\mathrm{m}$ Dy-168. All energies are in $\mathrm{MeV}$. The beta decay energy is an average of the mass ionnulae compiled by Haustein [Hau88]. Log ft's are in italics. The spin and parity assignments are discussed in the text. 
compiled by Haustein [Hau88]. The multipolarities of the 143- and 192-keV transitions were determined from intensity ratios with Ho $\mathrm{K} x$ rays $\left(\alpha_{\mathrm{K}}=5.4(4)\right.$ and $0.22(4)$, respectively) in coincidence spectra feeding each transition and the use of internal conversion coefficient tables [Røs78]. The multipolarity of the 43.8-keV transition could not be determined since $K$ conversion is energetically impossible and the Ho L x-ray energies were too low for the HPGe detector to measure, but was inferred from the intensity balances in the proposed decay scheme given in Figure 5.4. TAC spectra measured between the $193-\mathrm{keV}$ transition and $\beta$ - particles (in the beta telescope or plastic scintillator) indicated that the $192.5-\mathrm{keV}$ level was delayed. Figure 5.5 shows the decay fit of the TAC data for this delayed level and the measured half-life of $108(11)$ ns. The measured half-life of this level, as compared with the half-life calculated using the single-paricle model [Bla52] for an E2 transition and corrected for internal conversion was calculated as 5.0 Weisskopf units. The half-lives of all other levels were prompt ( $5 \mathrm{~ns}$ ). This is inconsistent with the measured M2 multipolarity for the $143.5-\mathrm{keV} \gamma$ transition which has a calculated half-life of $\sim 1.8 \mu$ s [Bla52] after correction for ICC.

The $1+$ spin and parity of the 192- and 630-keV levels in the decay scheme are the unly assignments that are consistent with their measured $\log f$ 's and ground state gamma branches. The negative parity assignments of the 143- and $187-\mathrm{keV}$ levels are consistent with the multipolarity assignments and their low beta feedings. A 0- or 1- spin assignment to the $187-\mathrm{keV}$ level is consistent with the level scheme, but a 0 - assigmment is inconsistent with the measured $\log f t^{\prime} \mathrm{s}$ of -7.2 to -8.1 for other $0+$ to 0 - beta transitions in this region [Led78]. The $143-\mathrm{keV}$ level's spin and parity must be the same as the $187-\mathrm{keV}$ level since they are connected by a M1 transition ( $\Delta \mathrm{l}=0$ or $1, \Delta \pi=$ no). From these arguments the spin and parity of the 


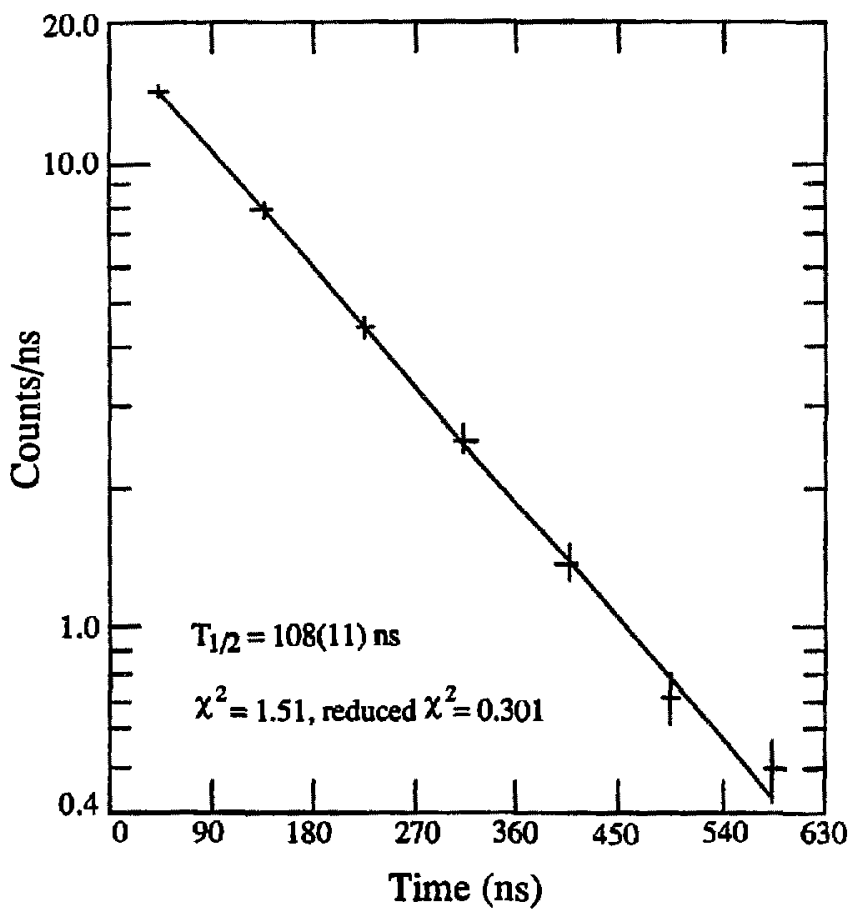

Figure 5.5. Single-component decay fit to the timing information berween beta particles (in the beta telescope or plastic scintillator) and 193-keV gamma transitions measured in the $A=168$ mass chain. 
143- and 187-keV levels are tentatively assigned as (1)-. The multipolarities of the 437-, 443-, 487-, and 630-keV transitions in Table 5.2 and Figure 5.4 3re based on the level spin and parity assignments. Ground state $\beta$-feeding would be inconsistent with the measured data and the parent and daughter spins and parities. Beta feeding to the $143-\mathrm{keV}$ level had a measured upper limit of $52(4) \%$; however, with all of the beta intensity (103(5)\%) assigned and the large percentage error on this beta branch, no beta feeding to this level is proposed.

\section{1b. ${ }^{168} \mathrm{Ho}^{\mathrm{m}}$ ISOMER}

When a short-lived activity was noted in the 1024-s nun data, the 512-s nun da: a vere analyzed more thoroughly than originally intended for the targetion source tests. The $H_{0} K_{\alpha 1}$ and $K_{\alpha 2} x$-ray singles activity again exhibited a two component decay behavior as shown in Figure 5.6. A weighted average of 132(4) $\mathrm{s}$ was determined for the half-life of the short-lived $x$-ray activities from both the 512-s and 1024-s runs. The intensities of the 132.s Ho $K_{\alpha 1}$ and $K_{\alpha 2} \times$ rays were determined by integrating the shor-lived activity of the decay curves. This was also done for the short-lived $x$-ray activities in the 1024-s run in Figure 5.2.

Two possible explanations of this observation are: a new ${ }^{168} \mathrm{Dy}$ isomer which predominantly $\beta$-decays ( ${ }^{168}$ Dy decay showed no growth and decay behavior) with an intemally converted $\gamma$ ray, or a new ${ }^{168} \mathrm{Ho}$ isomer which has an isomeric transition (IT) branch which is internally converted. Isomers of even-even isotopes are not expected in this region and this new activity is assigned to the new isomer ${ }^{168} \mathrm{Ho}^{\mathrm{m}}$ with a half-life of $132(4) \mathrm{s}$.

The decay intensity of this new isomer for decay by isomeric transition was determined by analyzing the growth and decay curves of its daughter activity, $3.0 \mathrm{~m}$ 


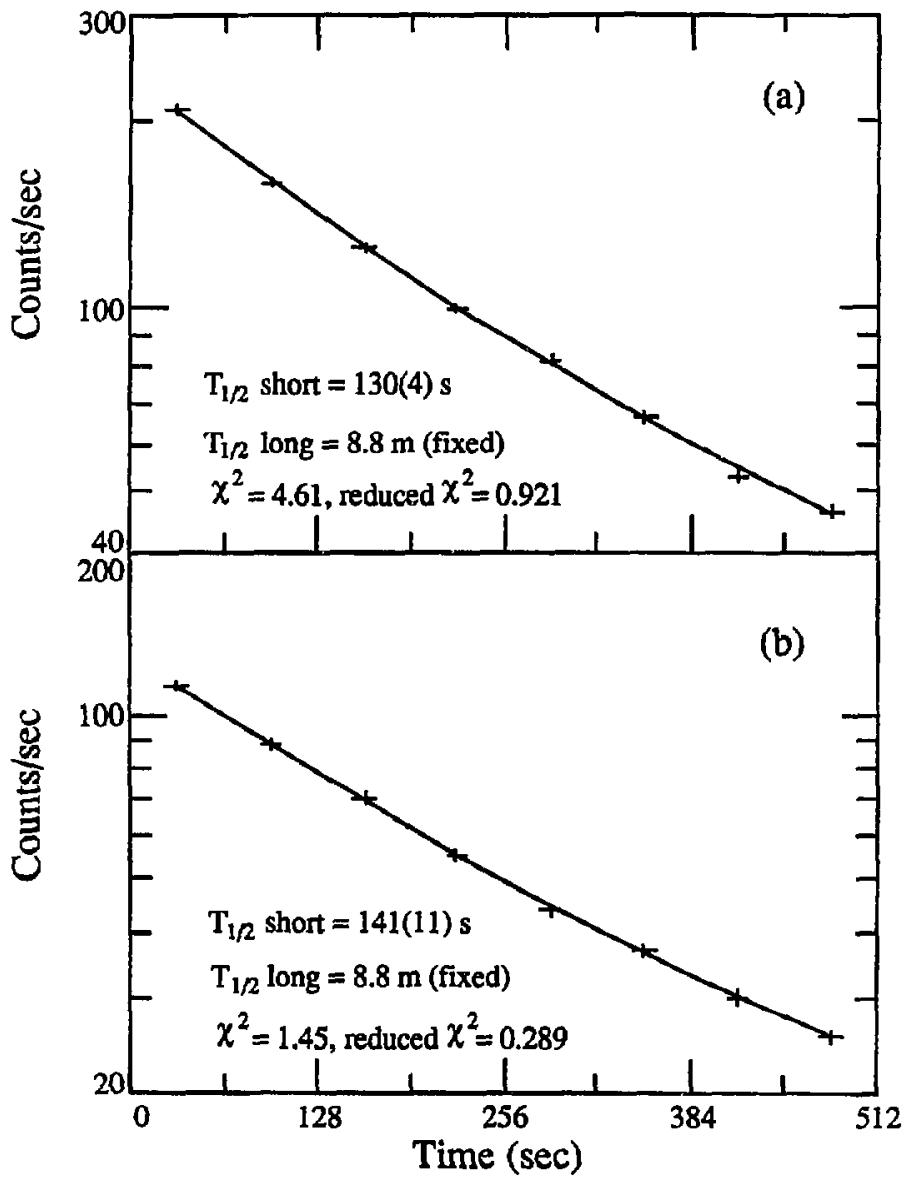

Figure 5.6. Two-component decay curves fit to the Ho $\mathrm{x}$-ray data for the 512-s run; (a) $\mathrm{Ho} \mathrm{K}_{\alpha 1}(47.5 \mathrm{keV})$, and (b) $\mathrm{Ho}_{\alpha 2}(46.6 \mathrm{keV})$. 
${ }^{168} \mathrm{Ho}$, as was done to determine the decay intensity of ${ }^{168} \mathrm{Dy}$. Figure 5.7 shows the decay curves of the 741 - and $821-\mathrm{keV}$ gamma rays of ${ }^{168} \mathrm{Ho}$ for the $512-\mathrm{s}$ run. A decay curve fit of two growth and decay components with one daughter's initial activity held fixed at zero and the long-lived parent and the daughter half-lives fixed at $8.8 \mathrm{~m}$ and $180 \mathrm{~s}$, yielded a $\sim 120 \mathrm{~s}$ short-lived parent half-life (fits shown in Figure 5.7). The ratio of the initial activities for the short- to long-lived parent activities was $\sim 11.2$ for the fit in Figure 5.7 during the 512-s nun. The intensity of the short-lived parent was integrated over the counting interval and corrected for the 741- and 821-keV emission probabilities [Led78]. This was also done for the shortlived parent in the 1024-s run growth and decay curves in Figure 5.3. An absolute Ho $\mathrm{K} x$-ray intensity of $0.133(10)$ was determined for the IT branch of $168 \mathrm{Hom}$ by dividing the $\mathrm{Ho}_{\mathrm{K}} \mathrm{x}$-ray intensity, (Ho $\mathrm{K}_{\mathrm{Q}_{1}}+\mathrm{K}_{\mathrm{\alpha 2}_{2}}$ intensity)/0.79, by the shortlived parent intensity (determined above) for both the 512-s and 1024-s runs.

The HPGe and $52 \%$ Ge detectors' singles data did not contain any gamma rays that decayed with this 132 -s half-life. All $y$ rays seen could be assigned to other $A=168$ activities or background radiations. The $\gamma$ rays of ${ }^{168}$ Ho were investigated very closely to determine that their intensities m*: shed those in the literature [Led78] and showed no $\beta$ - feeding from the ${ }^{168} \mathrm{Ho}^{\mathrm{m}}$ isomer. Based on the above facts, the $\beta$ - branch of this new isomer was determined to $50.5 \%$.

Having found only a half-life and $\mathbf{K} x$-ray intensity, we sought means to idendify the IT gamma ray. For internal conversion of Ho $\mathrm{K} x$ rays, the gamma transition in the decay of this new isomer must have an energy $257 \mathrm{keV}$. A high multipolarity is likely for the $\gamma$ transition depopulating the isomeric level in onder to a half-life as long as $132 \mathrm{~s}$. Also, any of the $\gamma$ rays in the decay of the isomer must be highly converted, or have energies less than $10 \mathrm{keV}$, or they would have beer. 


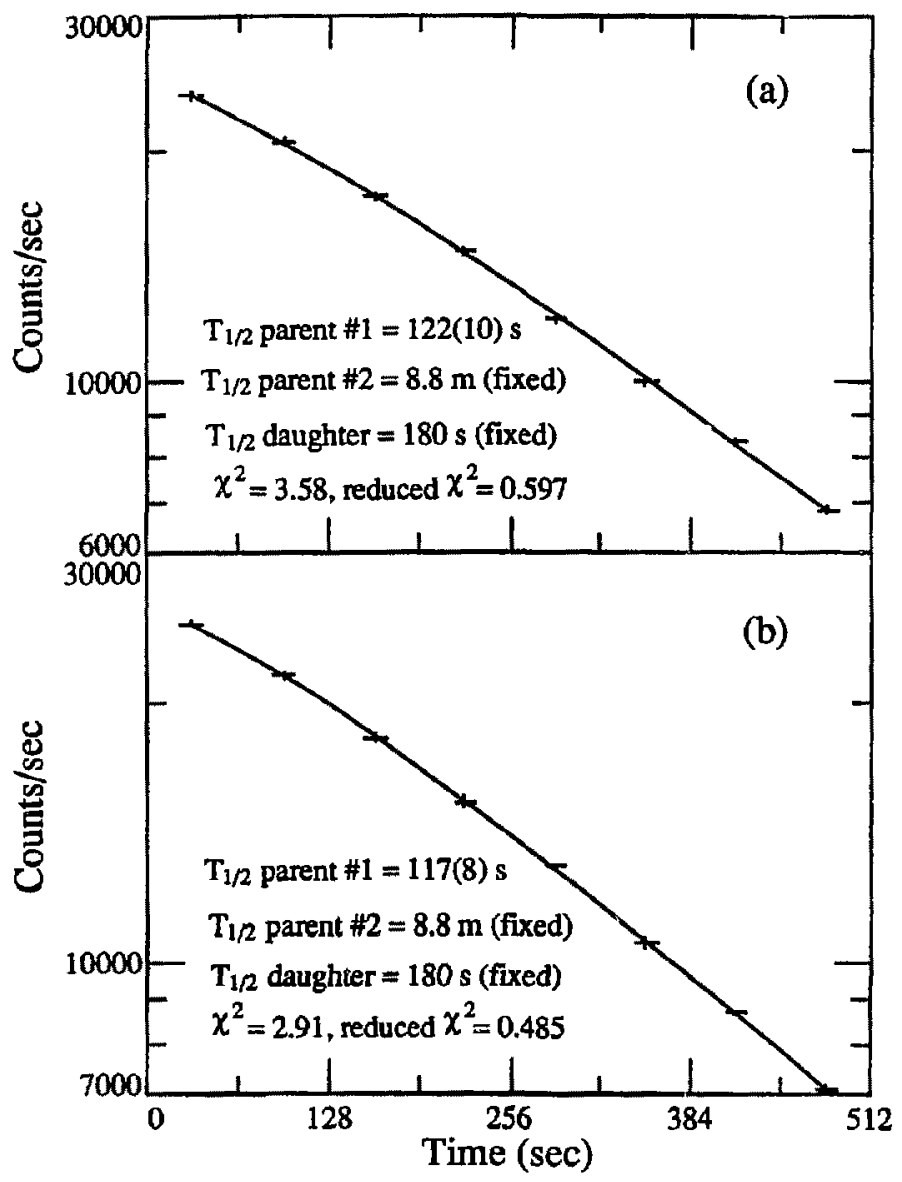

Figure 5.7. Calculated double growth and decay curves of ground and isomeric parent decay feeding one daughter actifity, $3.0-\mathrm{m} \mathrm{Ho}-168$, fit to the data from the 512-s nun; (a) 741-keV gamma-ray decay and (b) 821-keV gamma-ray decay. 
detected above the background in the singles data. Possible decay schemes are the following: a single, high-multipolarity, and highly-converted $\gamma$ transition ( $257 \mathrm{keV}$ ) decaying to the ground state of ${ }^{168} \mathrm{Ho}$; or a high-mulipolarity, highly-converted $\gamma$ transition that feeds one or more low energy ( $<10 \mathrm{keV}$ ) or highly-converted $\gamma$ transitions; or a high-multipolarity, low-energy ( $<10 \mathrm{keV}$ ) $\gamma$ transition that feeds a highly-converted $\gamma$ transition ( $257 \mathrm{keV}$ ). The prospect of finding the specifics of the last possibility was slim with the existing data due to the myriad of $\gamma$ energy and multipolarity combinations. The first two possibilities had one feature in common; a high-multipolarity, highly converted $\gamma$ ray with an energy $\geq 57 \mathrm{keV}$ that depopulates the isomeric level. A search for this gami.2a ray was performed by first calculating the energy and multipolarity of a $\gamma$ transition that would have a theoretical $\mathrm{K} x$-ray intensity equal to the measured value of $0.133(10)$. Figure 5.8 was prepared using internal conversion coefficient tables [R6s78] and dividing the $K$ conversion coefficient by the total conversion coefficient plus one (for the $\psi$-ray intensity) for each $\gamma$-ray energy and multipolarity. Transitions types E3, E4, M3, and M4 were the only multipolarities shown due to the level half-life requirements. The possible $\gamma$-ray energies $(59,91,127$, and $196 \mathrm{keV})$ for each transition type (M3, M4, E3, and E4, respectively) are indicated in Figure 5.8. Next, again using ICC tables [Rős78], theoretical absiolute $\gamma$-ray intensities were calculated, $\left(1+\alpha_{\text {tot }}\right)^{-1}$, for each of the four energies and multipolarities. The E3 and E4 transitions were not possible since their theoretical $\gamma$-ray intensities, $-6.0 \%$ and $\sim 6.8 \%$, respectively, indicate that the $\gamma$ ray would have been seen above the measured background. The theoretical Fray intensities of the 59-keV M3 and the 91-keV M4 $\gamma$ transitions, $-0.04 \%$ and $-0.03 \%$, respectively, are small enough for the $\gamma$ ray not to be measured. 


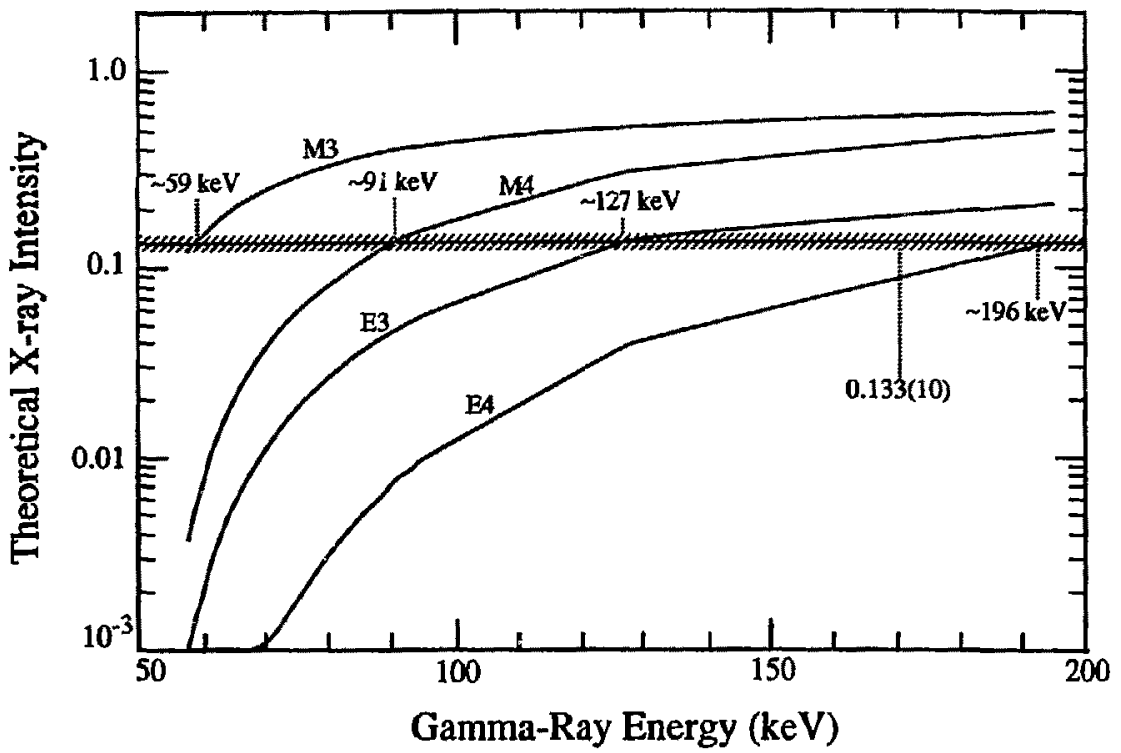

Figure 5.8. Theoretical $\mathrm{K} x$-ray intensities calculated for $\mathrm{M} 3, \mathrm{M} 4, \mathrm{E} 3$, and $\mathrm{E} 4$ gamma tansitions from ICC tables [Rös78] for a $Z \approx 67$ nucleus. Line at $x$-ray intensity $=0.133(10)$ is the measured value for the isomeric decay of Ho-168. 
Haif-lives of these $\gamma$ transitions were calculated using the single-particle model half-life formulae from Blatt and Weisskopf [Bla52]. They were corrected for their intemal conversion branches by multiplying the calculated half-life by the theoretical $y$ intensities calculated above. For a $Z=67$ and $A=168$ nucleus, a 59-keV M3 $\gamma$ transition would have a calculated half-life of $8.5 \mathrm{~s}$ and a 91-keV M4 $\gamma$ transition would have a 34-d half-life. The calculated half-life for the M4 transition was too large by four orders of magnitude, clearly in disagreement with M4 transitions, while the $M 3$ transition calculation was too short by a factor of $\sim 15$, well within measured distributions for this transition type [BlaS2]. Assuming a single IT M3 transition, a spin and parity of $6+$ is proposed for the isomeric level since the ground state of $16^{0--} .0$ has a $3+$ assignment [Hau71].

Similar isomerism is seen in another $\mathrm{N}=101$ nucleus, $1 / 2 \mathrm{Lu}$. This nucleus has an isomeric level that is depopulated by IT via a M3 gamma transition. Vaientin et al. [Val62] assigned 4 and I- spins and parities to the ground and isomeric states, respectively. They also proposed that these assignments arose from couplings of the $71^{\text {st }}$ proton level, $7 / 2+[404]$, with the $101^{\text {st }}, 1 / 2-[521]$, and 103rd, $5 / 2-[512]$, neutron levels. These levels, assignments and decay of $172 \mathrm{Lu}$ isomers are depicted in Figure 5.9a. These assignment of the 1- isomeric level consisting of the $7 / 2+[404]$ pioton and 5/2-[512] neutron levels has been further justified by the assignment of the ${ }^{174} \mathrm{Lu}$ (71 protons and 103 neutrons) ground state as 1-, also composed of the same proton and neutron level couplinge [One72]. Using the same single-particle model half-life formulae as above [Bla52], the calculated half-life after correction for intemal conversion for ${ }^{172} \mathrm{Lu}$ was $8.6 \mathrm{~s}$, too short by a factor of $\sim 26$. 


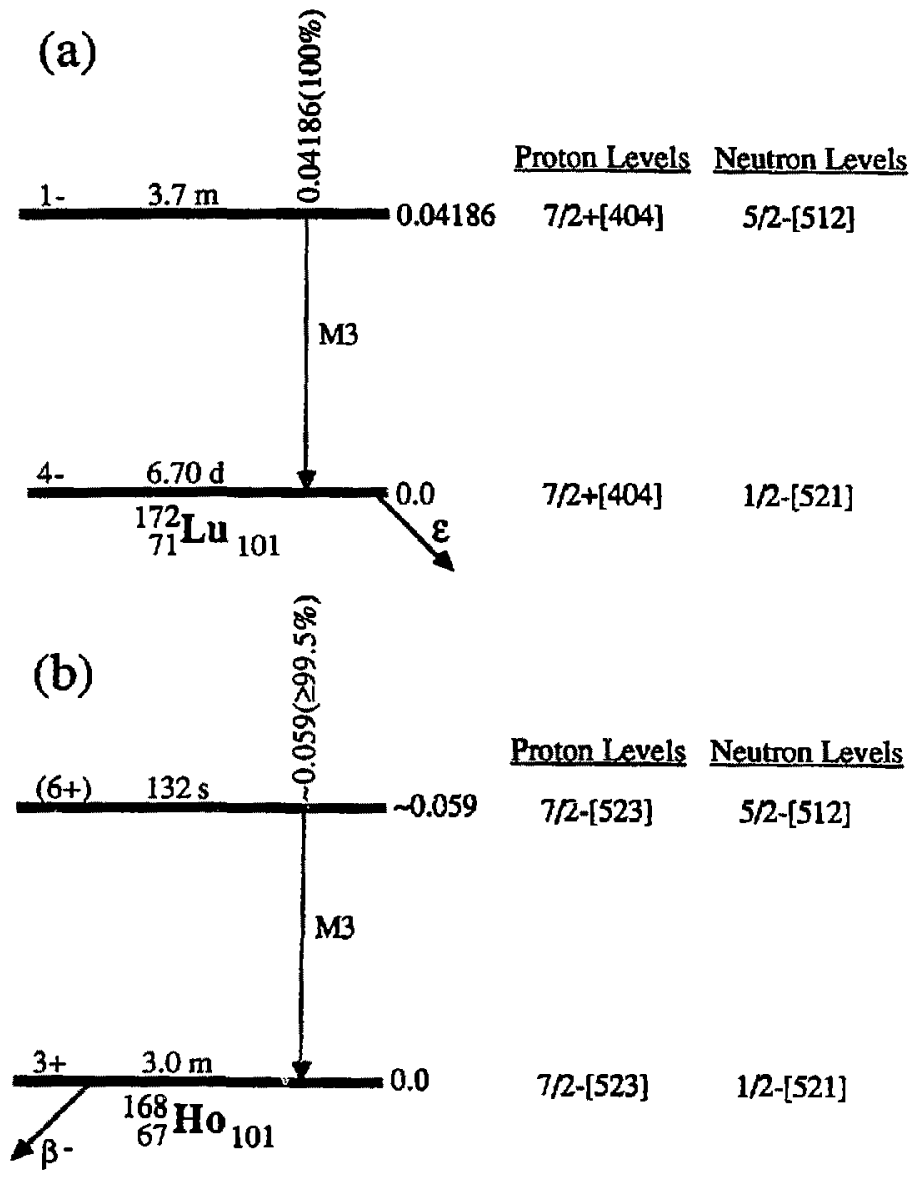

Figure 5.9. Level schemes of $\mathrm{N}=101$ isomers and their proton and neutron level couplings; (a) Lu-172 and Lu-172m, and (b) Ho-168 and proposed Ho-168m. 
If similar isomerism as ${ }^{172} \mathrm{Lu}$ is seen in ${ }^{168} \mathrm{Ho}$, the level scheme and assignments shown in Figure 5.9b result. The $7 / 2$-[523] spin and parity of the $67^{\text {th }}$ proton level has been well established for this region [Led78] and other evenmassed Ho isotopes [Kat78, Tuu78]. The ground state and isomeric levels result from couplings of the 7/2-[523] proton level with the $1 / 2-[521]$ and 5/2-[512] neutron levels. The assignment of the $6+$ isomeric state is further justified by the ground state assignment of ${ }^{170} \mathrm{Ho}$ (67 protons and 103 neutrons) as $6+$ consisting of couplings of the 7/2-[523] proton and 5/2-[512] neutron levels [Kat78, Tuu78]. It should be noted that the spin and parity assignments mentioned in this section are in agreement with predictions of Nilsson et al. [Ni169] using deformations predicted by Möller and Nix [Möl81].

The decay scheme in Figure 5.9b is the proposed scheme for the 132(4) s ${ }^{168} \mathrm{Ho}^{\mathrm{m}}$ isomer. Assignments of the spin, parity and energy of the isomeric level are uncertain due to the possibility of the $\sim 59 \mathrm{keV} \mathrm{M3}$ transition feeding another level which deexcites by a $\gamma$ ray with an energy too low $(<10 \mathrm{keV}$ ) to be detected by the measurement system or which is highly converted. However, due to similarities to ${ }^{172} \mathrm{Lum}$ decay, the proposed scheme in Figure $5.9 \mathrm{~b}$ is highly probable.

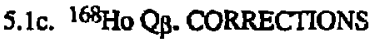

The accepted $Q_{\beta}$ - value for the decay of 3.0-m ${ }^{168} \mathrm{Ho}$ has been reported as 2.74(10) MeV [Kaw73]. This result was obtained from endpoint measurements of $\beta$ - particles coincident with gamma rays depopulating the 821 -ke $\mathrm{V}$ level in $168 \mathrm{Er}$. Recalculations from their data indicated an average endpoint energy of $1.90(9) \mathrm{MeV}$ for $\beta$ - particles in coincidence with 80,741 , arid $821-\mathrm{keV}$ gamma rays. Their reported $Q_{p}$ - value was determined using only the endpoint energy of the $821-\mathrm{keV}$ 
coincident $\beta$ - particles, $1.91(10) \mathrm{MeV}$. The beta endpoint of ${ }^{168} \mathrm{Ho}$ had also been measured prior to the results above [Kaw73] as $\sim 2.2 \mathrm{MeV}$ (with no reported error) [Tak61], however, this value was obtained with no gamma gating.

During the determination of the $\beta$ telescope efficiency, section 4.3, endpoint energy problems were noted. The endpoint energies in Figures 4.2 and 4.3, $2.10(6)$ and $2.10(6) \mathrm{MeV}$, for $\beta$ - particles coincident with 741- and 821-keV gamma rays , w not agree with the accepted value [Kaw73], but are similar to the value measured by Takah;:shi et al. [Tak61]. An extensive study of the beta endpoints for the decay of ${ }^{168} \mathrm{Ho}$ was undertaken. Beta endpoints were determined for $\beta$ feedings to levels at 821.1, 895.7, and 994.7 keV [Hau71]. These levels were chosen for their large $\beta$-feedings and small feedings from more excited levels of ${ }^{168} \mathrm{Er}$. Gates were set on the two strongest gamma transitions depopulating each level and their corresponding backgrounds in the $52 \%$ Ge detector. The beta spectra coincident with these gates were obtained. Beta spectra were generated by adding the two $\gamma$-gated $\beta$ telescope spectra and subtracting the background-gated $\beta$ telescope spectra for each level. Fermi-Kurie plots were calculated for each of the three beta spectra and their corresponding endpoints determined. Figures 5.10, 5.11 , and 5.12 show the gamma-coincident $\beta$ - spectra, corresponding Fermi-Kurie plots and $\beta$-endpoints. The results of this analysis are summarized in Table 5.3 which contains the level energy, $\%$ gates used, measured endpoints, and the calculated Qp. values. The beta endpoint found by Kawade et al. [Kaw73] for the 821-keV level is also listed in the table. A weighted average of 2.93(3) MeV was determined from the three $\mathrm{Q}_{\beta}$ - values in Table 5.3, which gives 2.93(3) MeV as the QB- value for the decay of $3.0-\mathrm{m}^{168} \mathrm{Hog}$. Comparisons of revised $\log f$ values 


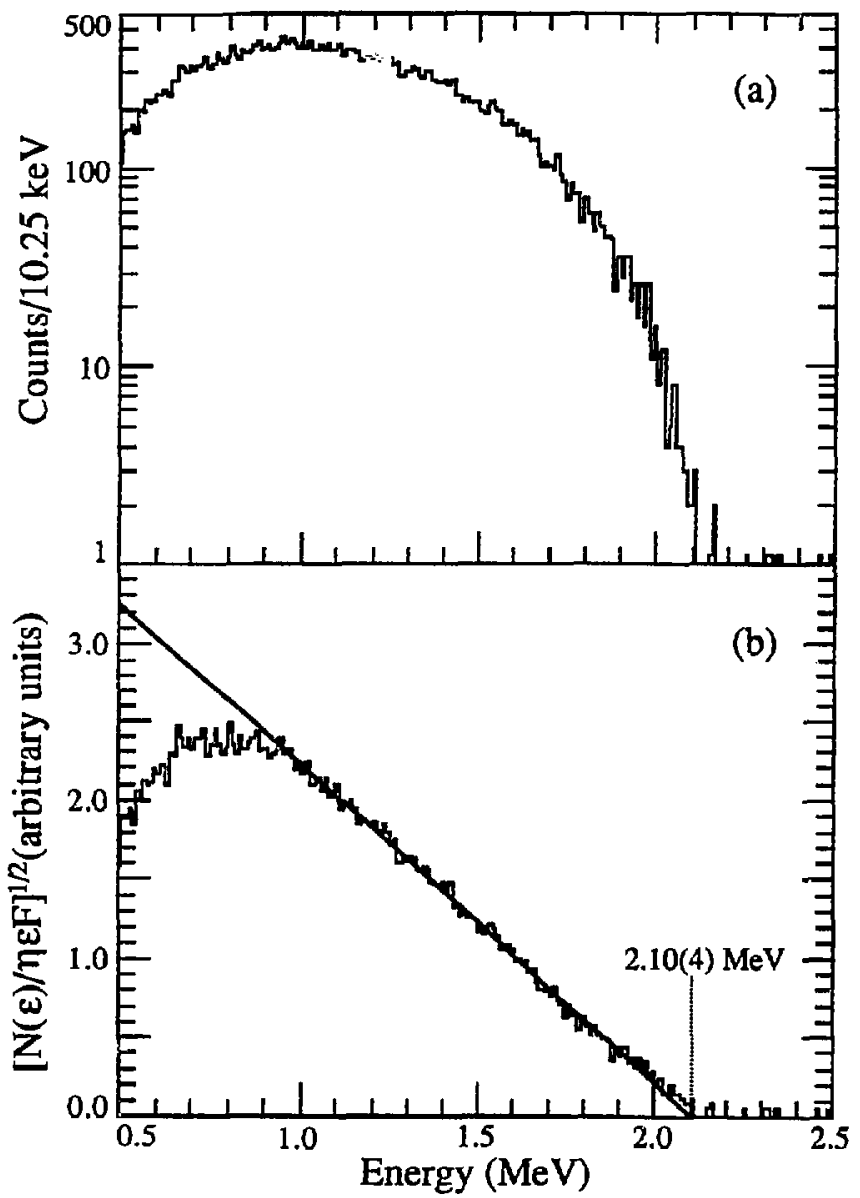

Figure 5.10. Beta decay data for $\beta$-particles measured in the beta telescope coincident with gamma rays depopulating the 821-keV level (see Table 5.3) in the decay of 3.0-m Ho-168; (a) actual coincident beta spectrum and (b) Fermi-Kurie plot and endpoint energy. 


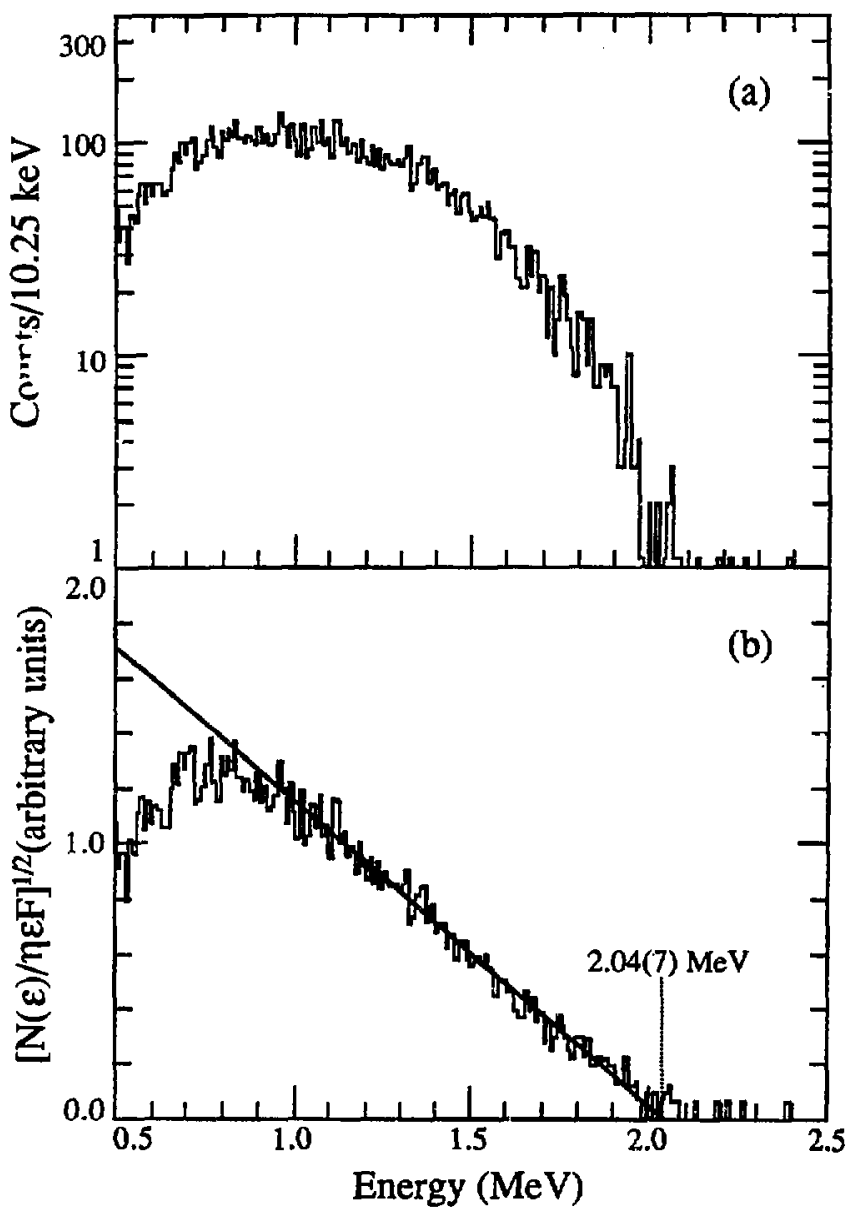

Figure 5.11. Beta decay data for $\beta$-particles measured in the beta telescope coincident with gamma rays depopulating the 896-keV level (see Table 5.3) in the decay of 3.0-m Ho-168; (a) actual coincident beta spectrum and (b) Fermi-Kurie plot and endpoint energy. 


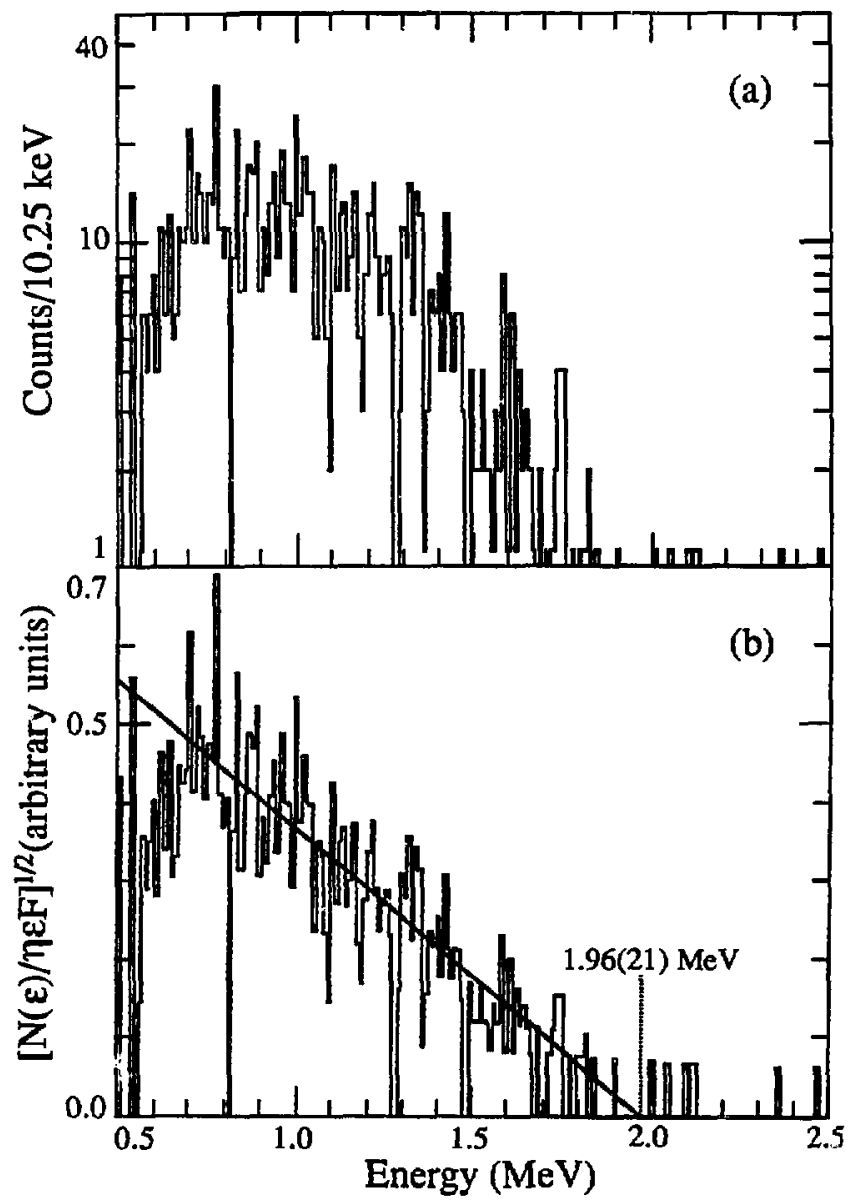

Figure 5,12. Beta decav data for $\beta^{-}$particles measured in the beta telescope coincident with gamma rays depopulating the 995-keV level (see Table 5.3) in the decay of 3.0-m Ho-168; (a) acrual coincident beta spectrum and (b) Fermi-Kurie plot and endpoint energy. 
Table 5.3. Beta endpoint data for $Q_{\beta}$ - value determinations for the decay of $3.0 \mathrm{~m}^{168} \mathrm{Ho}$, and the previously measured endpoint energy.

\begin{tabular}{|c|c|c|c|c|}
\hline $\begin{array}{c}\text { Level Energy } \\
(\mathrm{kev})\end{array}$ & $\begin{array}{l}\text { Yray Gates } \\
\text { Useci(keV) }\end{array}$ & $\begin{array}{c}\text { Measured } \beta \text { - } \\
\text { Endpoint (MeV) }\end{array}$ & $\begin{array}{l}\text { Endpoint from } \\
\text { [Kaw73] (MeV) }\end{array}$ & $\begin{array}{l}\text { Calculated } Q_{\beta}- \\
\text { Value }(\mathrm{MeV})\end{array}$ \\
\hline 821.1 & 741,821 & $2.10(4)$ & $1.90(9)$ & $2.92(4)$ \\
\hline 895.7 & 632,816 & $2.04(7)$ & - & $2.94(8)$ \\
\hline 994.7 & 731,915 & $1.96(21)$ & - & $2.96(21)$ \\
\hline
\end{tabular}

Table 5.4. Comparison of old $\log f t$ values [Led78] for the $\beta$ decay of 3.0-m ${ }^{168} \mathrm{Ho}$ with revised values calculated with new Qp. value 2.93(3)MeV.

\begin{tabular}{|c|c|c|c|}
\hline 168 Er level (keV) & $\beta-$ in $\%(\%)^{\mathrm{a}}$ & Old $\log f^{\mathrm{a}}$ & Revised $\log f t$ \\
\hline 821.1 & 71 & 5.3 & 5.4 \\
895.7 & 18 & 5.8 & 6.0 \\
994.7 & 2.0 & 6.6 & 6.8 \\
1541.4 & 2.6 & 5.9 & 6.2 \\
1569.5 & 0.15 & 7.1 & 7.4 \\
1848.2 & 0.58 & 6.1 & 6.4 \\
1915.3 & 0.13 & 6.6 & 7.0 \\
1930.2 & 0.33 & 6.2 & 6.5 \\
1994.6 & 0.19 & 6.3 & 6.7 \\
2193.0 & 1.7 & 4.9 & 5.4 \\
2254.7 & 0.38 & 5.3 & 5.9 \\
2424.9 & 0.46 & 4.6 & 5.4 \\
2484.6 & 0.072 & 5.1 & 6.0 \\
\hline
\end{tabular}

a Values are from [Led78]. 
calculated using the new $Q_{\beta}$. value with $\log f$ is [Led78] for the old $Q_{\beta}$. value are listed in Table 5.4.

\section{2. ${ }^{169}$ Dy - NEW ISOTOPE}

The $\mathrm{A}=169$ mass chain was studied using ${ }^{170} \mathrm{Er}$ ions with the standard OASIS target/ion source geometry (capillaries, W endplate, no window) or sources with W electroplated on Ta endplates. Natural erbium or ${ }^{170} \mathrm{Er}$ enriched $(\sim 1.7 \mathrm{~g}$ ${ }^{170} \mathrm{Er}$ strip spot-welded to nanural erbium closest to the plasma region of the SuperHILAC ion source) ion sources were used as the source material for the SuperHILAC to deliver beam intensities of 35 to 100 particle nanoamperes on target for -57 hours. This experiment was performed to search fol the new isotope ${ }^{169} \mathrm{Dy}$. Using the mass prediction compilation [Hau88], a simple average Q. value of $3.2 \mathrm{MeV}$ was calculated for the decay of this isotope. Half-lives of $38 \mathrm{~s}$ [Kla84] and $64 \mathrm{~s}$ using the GTBD code [Tak88] and the $\mathrm{Q}_{\beta}$-value above were predicted for this isotope. A tape cycle time of $160 \mathrm{~s}$ was chosen to maximize the measured activity. A summary of half-lives, decay modes, and $Q$ values of th.e other $A=169$ isobars [Bro86] that could be separated by OASIS is listed in Table 5.5. Actually, ${ }^{169} \mathrm{Hf}$ was not surface ionized well ( $10^{-4}$ efficiency compared to $\mathrm{Lu}$ isotopes) in the OASIS ion source, due to its high first ionization potential $(7.0 \mathrm{eV}$ versus $5.4 \mathrm{eV}$ for Lu).

High-energy singles $\beta$-particles were measured in the beta telescope with energies beyond the $\beta$ endpoints of the istoiopes listed in Table 5.5 and are shown in Figure 5.13a. Because $\beta+$ maximum energies are $1.022 \mathrm{MeV}$ less than the decay energy, ${ }^{169} \mathrm{Hf}$ and ${ }^{169} \mathrm{Lu}$ endpoints were ruled out. The most energetic beta endpoint, $2.03 \mathrm{MeV}$, for ${ }^{169} \mathrm{Ho}$ results from a small, $<\%$, beta branch to a 91.9 


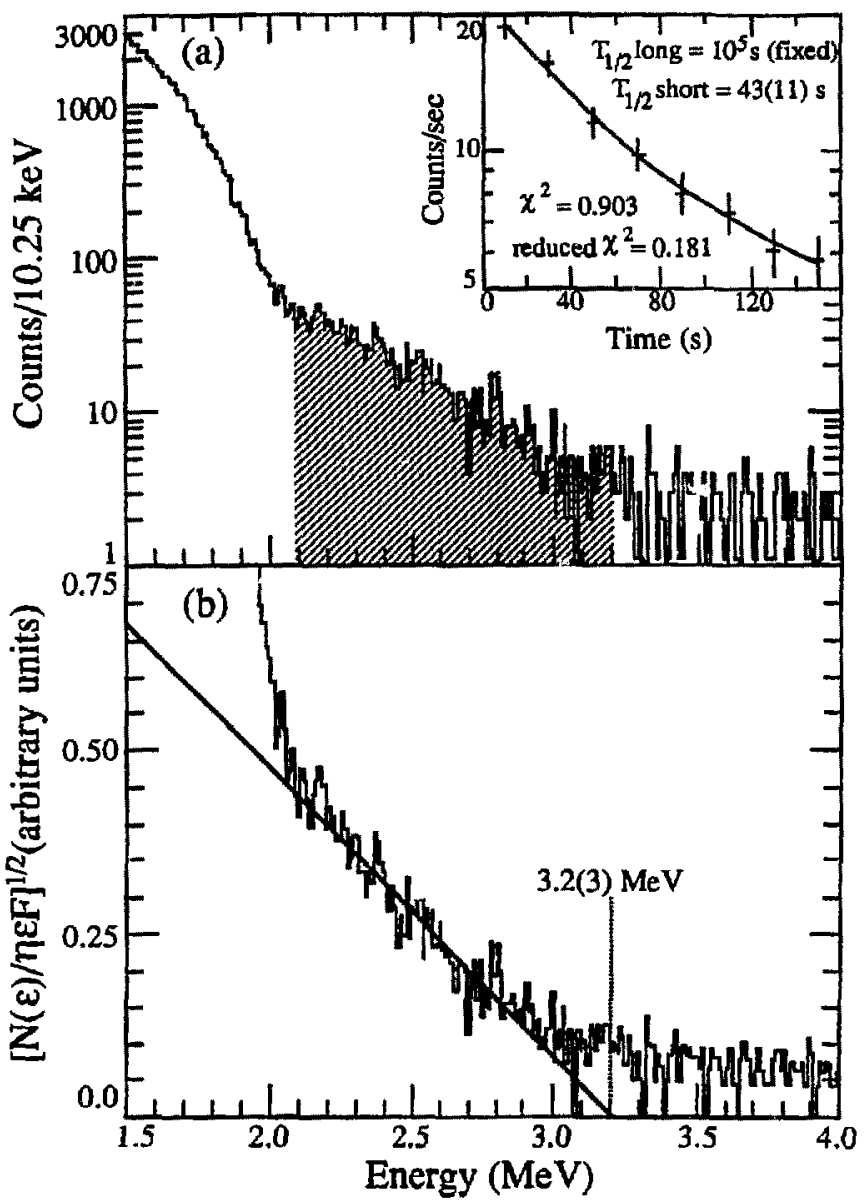

Figure 5.13. Beta decay data for $A=169$ mass chain; (a) beta particle spectrum measured in the beta telescope and (b) Fermi-Kurie plot and 3.2(3) MeV endpoint. The inset in (a) shows the two-component decay fit of the beta intensity from 2.1 to $3.2 \mathrm{MeV}$ (shaded porrion of the spectrum). 
Table 5.5. Summary of $A=169$ isobar information [Bro86] for nuclei that can be separated in OASIS. ${ }^{169} \mathrm{HF}\left(-10^{-4}\right.$ ionization efficiency relative to the other elements) is listed since some of this isobar might have been separated.

\begin{tabular}{|c|c|c|c|}
\hline Isotope & Half-life & Decay Mode & Q value (MeV)a \\
\hline${ }^{169} \mathrm{Hf}$ & $3.24 \mathrm{~m}$ & EC, $\beta^{+}$ & $3.35(10)$ \\
$169 \mathrm{Lu}$ & $1.42 \mathrm{~d}, 2.7 \mathrm{~m}^{\mathrm{b}}$ & EC, $\beta^{+}, \mathrm{TT}$ & $2.293(3)$ \\
$169 \mathrm{Yb}$ & $32.0 \mathrm{~d}, 46 \mathrm{~s}^{\mathrm{c}}$ & $\mathrm{EC}, \mathrm{IT}$ & $0.908(4)$ \\
$169 \mathrm{Tm}$ & stable & - & - \\
${ }^{169} \mathrm{Er}$ & $9.40 \mathrm{~d}$ & $\beta-$ & $0.3509(14)$ \\
${ }^{169} \mathrm{Ho}$ & $4.7 \mathrm{~m}$ & $\beta-$ & $2.125(20)$ \\
$169 \mathrm{Dy}$ & $-38 \mathrm{~s}, \sim 64 \mathrm{~s}^{\mathrm{d}}$ & $\beta-$ & -3.2 \\
\hline
\end{tabular}

a $Q$ value reported is for ground state decay.

b $169 \mathrm{Lu}$ has an IT decaying isomer at $24.2 \mathrm{keV}$. Ground state halflife is listed first.

c ${ }^{169} \mathrm{Yb}$ has an IT decaying isomer at $29.0 \mathrm{keV}$. Ground state halflife is listed first.

d Listed $\mathrm{T}_{1 / 2}$ values are predictions, [K]a84] and [Tak73] respectively.

- $Q$ value is an average from mass tables compiled in [Hau88].

keV level in $169 \mathrm{gr}$ [Hau71]. Beta particles with energies above the $2.03 \mathrm{MeV}{ }^{169} \mathrm{Ho}$ endpoint were seen clearly above the background (similar to the 3.5 to $4.0 \mathrm{MeV}$ region in Figure 5.13a). The inset to Figure 5.13a shows the two-component decay fit of the background-subtracted beta intensity between 2.1 and $3.2 \mathrm{MeV}$ 'shaded region), with the long component held fixed at a very long half-life, $10^{5} \mathrm{~s}$, to represent background that wasn't completely subtracted. A half-life of $43(11) \mathrm{s}$ was obtained for the shor-lived activity, which again does not agree with any of the 
listed radiations in Table 5.5. A Fermi-Kurie plot of the $\beta$ particles measured is shown in Figure 5.13b. A $\beta$-endpoint of 3.2(3) $\mathrm{MeV}$ was obrained from fitting the region from 2.1 to $2.9 \mathrm{MeV}$. Gumma coincidence spectra with these energetic $\beta$ particles produced only background radiations. To check the $\beta$ telescope energy calibration $\gamma$ gates were set on $\gamma$ radiations depopulating the $853-\mathrm{ke} V$ level in the decay of ${ }^{169} \mathrm{Ho}$ and a beta endpoint of $1.3(1) \mathrm{MeV}$ was otrained, in excellent agreement with $1.3 \mathrm{MeV}$ found in the literature [Hau71].

Close inspection of the $\gamma$-ray and $x$-ray singles data yielded only one shortlived gamma ray at $1578.2(4) \mathrm{keV}$. It decayed with a half-life of $35(11) \mathrm{s}$ as shown in the two-component decay fit in Figure 5.14. Holding the long-component fixed at a long half-life, $10^{5} \mathrm{~s}$, produced the best chi-squared value for this decay. No Ho $\mathrm{K} x$ rays were observed and all other $\gamma$ rays measured were longer lived. The beta particle spectrum gated by this $1578-\mathrm{keV} \boldsymbol{\gamma}$ ray is shown in Figure 5.15. Statistics were too poor to obtain a beta endpoint, but visual inspection of the spectrum indicates an endpoint of $-1.5 \mathrm{MeV}$, which is consistent with a total decay energy of $3.2 \mathrm{MeV}$. The time histogram of the 38 beta particles in coincidences with $1578-$ $\mathrm{keV} \gamma$ rays is shown in Figure 5.16a. A maximum likelihood fit of these data is shown in Figure 5.16b. (Details on the maximum likelihood formulae and computer code used are given in Appendix B.) The likelihood calculation reached a maximum at $58 \mathrm{~s}$ and dropped to $50 \%$ of this maximum ( $68 \%$ confidence limits) at 42 and $90 \mathrm{~s}$ which gave a half-life of $58\left(\begin{array}{c}+32 \\ -16\end{array}\right) \mathrm{s}$.

These results indicated a beta decaying isotopes with a 39(8) s half-life, obtained from a weighted average of the high-energy $\beta$ - particle and 1578-keV $\gamma$ ray half-lives, which decays to the ground state, with a maximum $\beta$ - energy of 3.2(3) $\mathrm{MeV}$, or w a $1578.2(4)-\mathrm{keV}$ level which deexcites directly to the ground state. 


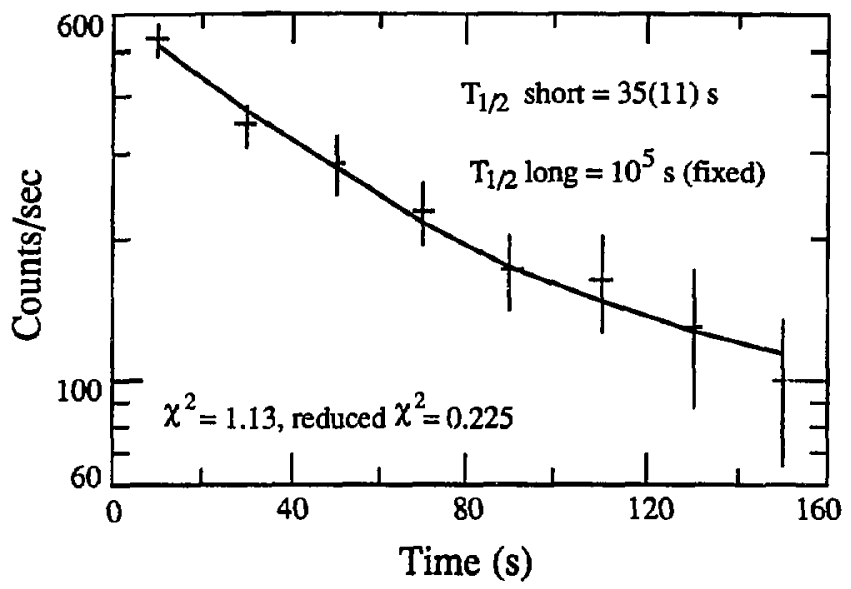

Figure 5.14. Two-component decay curve fit to the intensity distribution of the $1578.2(4) \mathrm{keV}$ gamma rays measured in the $52 \%$ Ge detector for the $A=169$ mass chain.

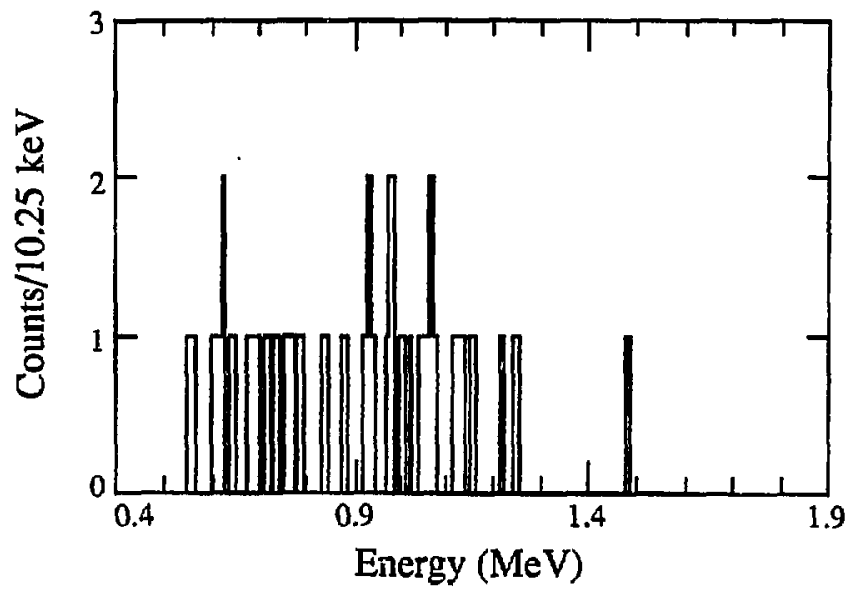

Figure 5.15. Beta particle spectum measured in cuincidence with $1578-\mathrm{keV}$ gamma mys in the $A=169$ mass chain. 


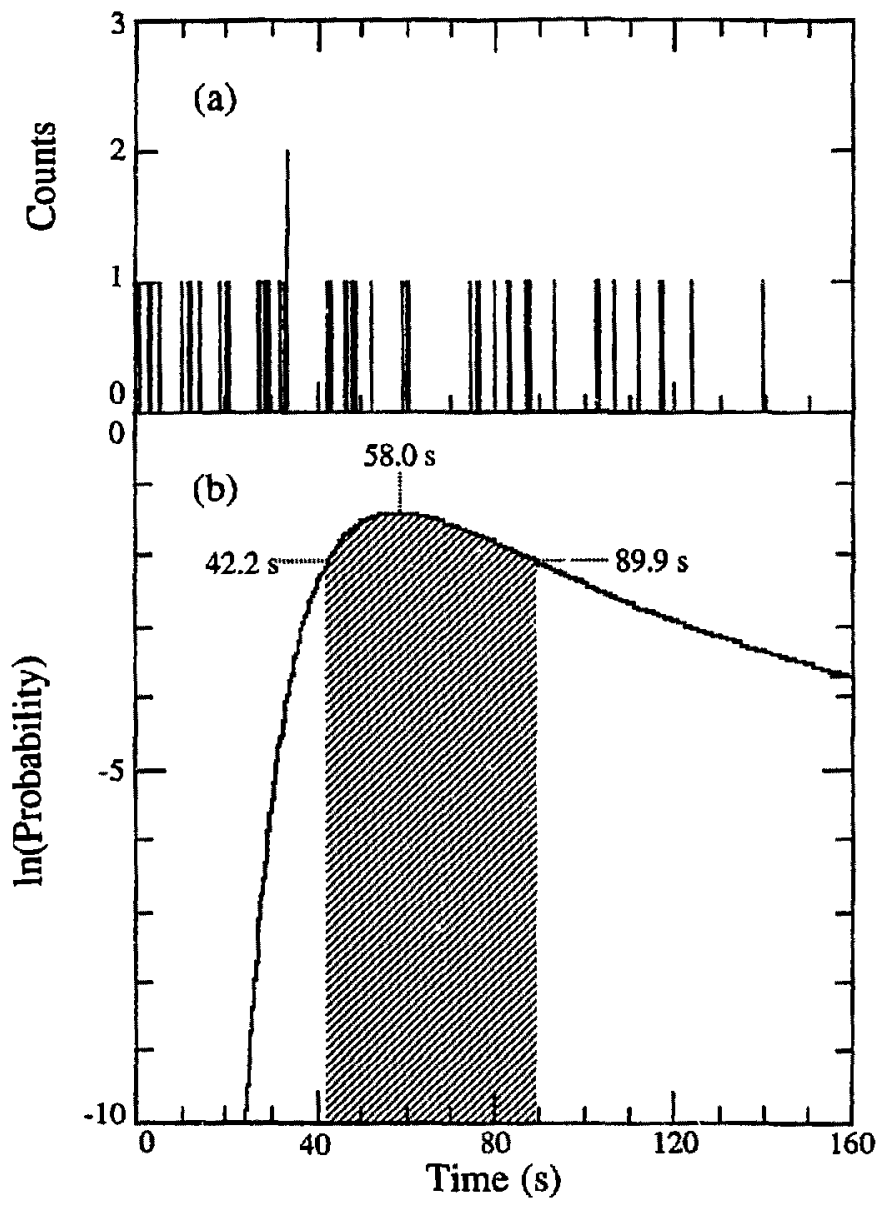

Figure 5.16. Time information of the beta particles measured in coincidence with 1578-keV gamma rays in the $A=169$ mass chain; (a) time distribution and (b) maximum likelihood calculation showing the probabilty of the time being the correct half-life to fit the time distribution. Shaded region shows where the probability drops to 50\% of the maximum value ( $68 \%$ confidence limits). 
Based on the agreement of the measured beta endpoint to the predicted $Q_{\beta}$ - value ror ${ }^{169}$ Dy and of the measured half-life with the predicted values, the discovery of the new isotope ${ }^{169} \mathrm{Dy}$ is suggested. The intensity of the ground state beta branch was determined by calculating a $\beta$ - spectrum with a $3.2 \mathrm{MeV}$ endpoint as was done for the $\beta$ telescope calibration, section 4.3. This calculated spectrum was normalized to the measured $\beta$ spectrum in the 2.3 to $3.0 \mathrm{MeV}$ interval. The measured and calculated $\beta$ spectra are shown in Figure 5.17. The integral of the calculated $\beta$ spectrum was corrected for the $\beta$ telescope efficiency to determine the ground state $\beta$ - intensity. The $1578 \mathrm{keV} \gamma$-ray's intensity was determined from the $52 \% \mathrm{Ge}$ detector because the statistics were poor in the $24 \%$ Ge detector (low efficiency at this energy, see Figure 4.1) and summing was not a problem since there were no other gamma rays in coincidence. Ground state and 1578-keV beta branches of 0.78 and 0.22 , respectively, were determined. Estimated errors on these quantities are $-20 \%$ due to uncert. .ies in the method to determine the ground state $\beta$ intensity and possible missed weak $\gamma$ rays.

The tentative decay scheme proposed for ${ }^{169} \mathrm{Dy}$ is shown in Figure 5.18 . This scheme is consistent with the lack of $\mathrm{x}$ rays since there would be very little internal conversion. The assignments of the $103^{\text {nd }}$ neutron (odd particle in ${ }^{169} \mathrm{Dy}$ ) and the $67^{\text {h }}$ proton (add particle in ${ }^{16 \%} \mathrm{Ho}$ ), 5/2-[512] and 7/2-[523], respectively, are well established for this region [Led78 and discussion in secrion 5.1b]. The measured $\log f t=5.4$ for the ground state beta branch is consistent with the $\log f t=$ 5.8 for the 7/2-[523] proton to $5 / 2-[512]$ neutron transition reported in the decay of ${ }^{167} \mathrm{Ho}$, but it is lower than the $\log f t=6.4$ for the $5 / 2-[512]$ neutron to $7 / 2-[523]$ proton transition previously measured in ${ }^{171} \mathrm{Er}$ decay [Led78]. The $\log _{\mathrm{g}} f t=4.8$ for the $\beta$-feeding to the $1578-\mathrm{keV}$ level is faster than any known $\beta$ transition involving 

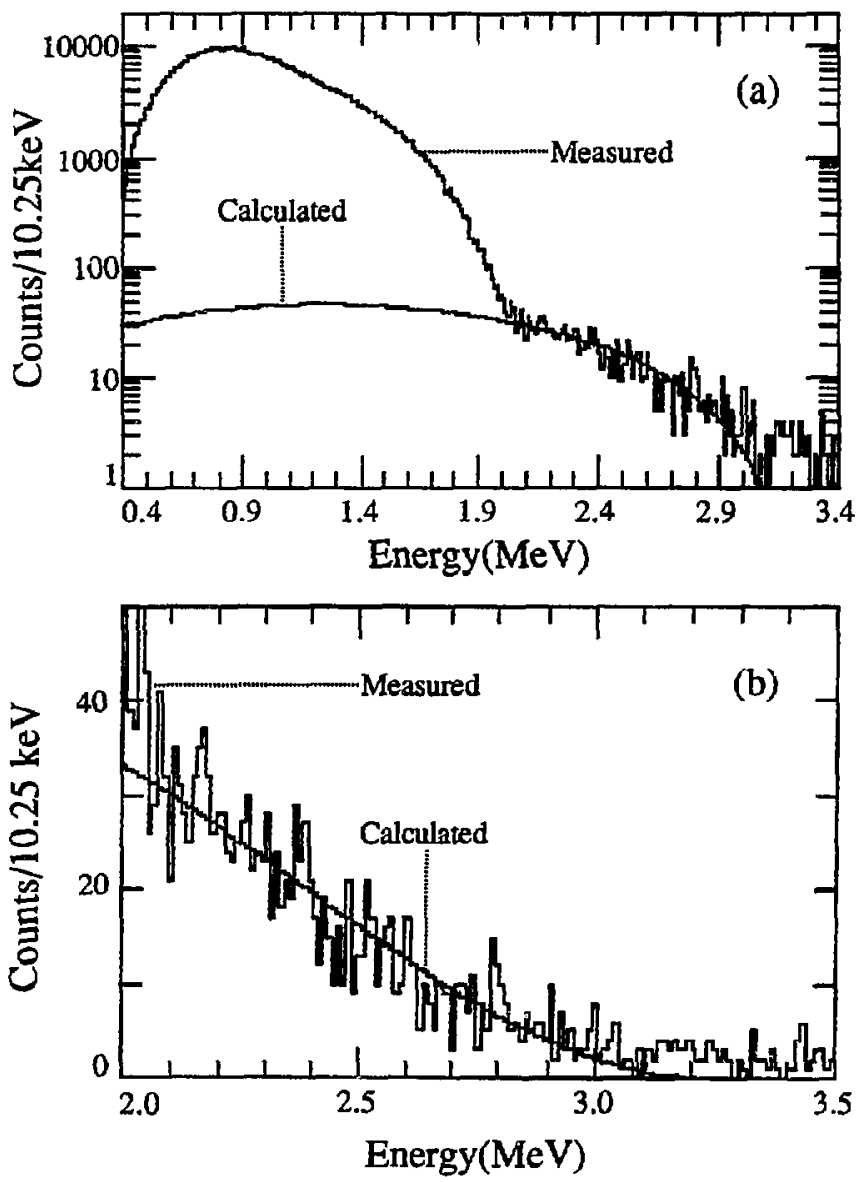

Figure 5.17. Fit of the measured $A=169$ beta spectrim with the calculated $\beta$ spectrum with a $3.2 \mathrm{MeV}$ endpoint, normalized to the 2.1 to $2.9 \mathrm{MeV}$ interval; (a) entire spectrum and (b) the 2.0 to $3.5 \mathrm{MeV}$ region of the spectrum. 


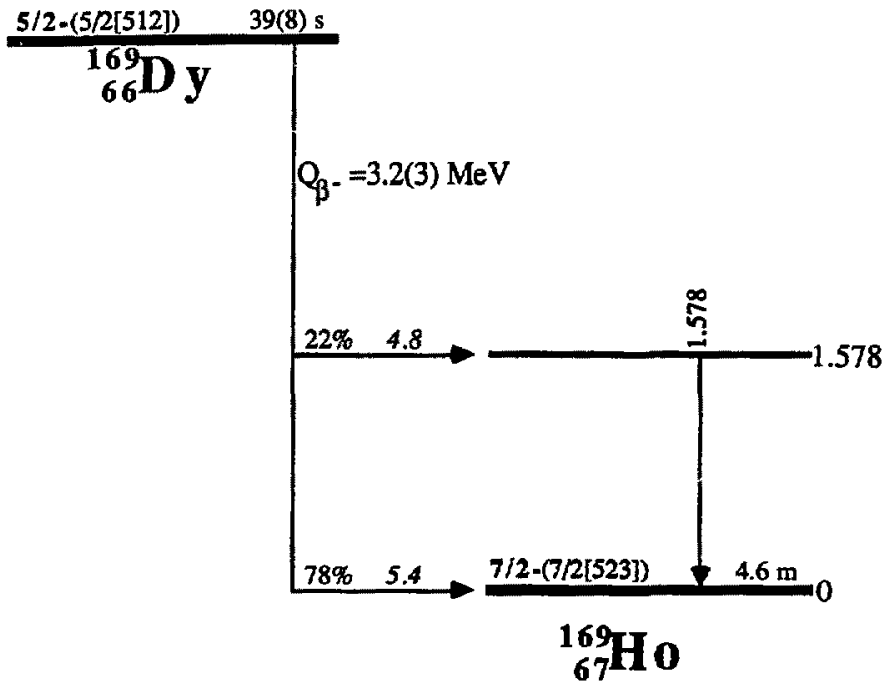

Figure 5.18. Proposed tentative decay scheme for $39 \mathrm{~s} D y-169$. Logft's are in icalics. All energies are in $\mathrm{MeV}$. The $\mathrm{Q}$ value was determined in the experiment. 
the 5/2-[512] neutron level. A three quasiparticle state could be a possible assignment of this level composed of $7 / 2-[523]$ proton and 5/2-[512] neutron particle states and a 5/2-[523] neutron hole state. This assignment would have a resulting $7 / 2$ - spin and parity which permits allowed beta feeding ( $\Delta \mathrm{I}=1, \Delta \pi=n$ ) and strong ground state $\gamma$ feeding. The assignment of the 5/2-[512] neutron level to the $39-\mathrm{s}{ }^{169}$ Dy parent and the $7 / 2$-[523] proton orbital to the measured ${ }^{169} \mathrm{Ho}$ ground state are tentatively proposed and in agreement with nuclear level predictions of Nilsson er al. [Nil69]. The 1578-keV level has been left unassigned.

\section{3. ${ }^{171}$ Ho DECAY SCHEME}

The $A=171$ mass chain was studied in both the ${ }^{176} \mathrm{Yb}$ and ${ }^{170} \mathrm{Er}$ beam experiments. Natural ytterbium ion sources were used in the SuperHIILAC to deliver beam intensities of 18 to 27 particle nanoamperes of $176 \mathrm{Yb}$ ions on target for -30 hours. This experiment was performed to look for ${ }^{171}$ Ho which was thought to be an undiscovered isotone. Half-life predictions were 72.4 [Kla84] and $84.7 \mathrm{~s}$ [Tais73], so a tape cycle time of $256 \mathrm{~s}$ was chosen. The production rate for this ( $3 p 2 n$ ) out reaction channel was extremely low, but Er $\because$ x rays were seen to decay on-line with a half-life of $\sim 50 \mathrm{~s}$. At this point, a tape cycle time of $64 \mathrm{~s}$ was used to try to maximize the coincidence infommation. Due to limited ${ }^{176} \mathrm{Yb}$ beam time and the low production rate, very linle data on ${ }^{171}$ Ho decay were actually obtained. After the experiment, it was found in two annual reponts from GSI [Ryk84] and Mainz [Ryk88] that the $A=171$ mass chain had been studied before. Both papers refer to the same experiment performed by a GSI collaboration where they reported a 45(5) s component in the electron spectrum reconded with a thin plastic scintillator and weak erbium $\mathrm{K}$ x rays in coincidence with them. The authors made a 
centative assignment of this activity to either the $\beta$ - decay of the new isotope ${ }^{171} \mathrm{Ho}$ or the $\mathrm{IT}$ decay of the new isomer ${ }^{171} \mathrm{E}^{\mathrm{m}} \mathrm{m}$, both decaying by strongly converted transitions. Discrimination between the two tentative assignments was not possible from their data since the plastic scintillawor used could not distinguish between high energy beta particles and conversion electrons.

The data from the $176 \mathrm{Yb}$ beam experiments were analyzed, but the low production rate yielded little information. Erbium $\mathrm{K} \times$ rays were detected which decayed with a half-life of $49(5)$ s. These $x$ rays were seen in coincidence with $\beta$ particles in the $\beta$ telescope having energies up to $-2.5 \mathrm{MeV}$ and in weak coincidence with the known 198- and 280-keV $\gamma$ transitions in ${ }^{171} \mathrm{Er}$ [Shi84]. These results unambiguously assigned the new activity detected in this research and in previously reported results [Ryk84, Ryk88] to the $\beta$ - decay of ${ }^{171} \mathrm{Ho}$. These results have been published [Cha89]. Later in 1989, Rykaczewsti et al. formally published their $A=171$ mass chain findings [Ryk89]. They assigned the $49 \mathrm{~s}$ activity to ${ }^{171} \mathrm{Ho}$ using half-life and systematic arguments to eliminate the ${ }^{171} \mathrm{Er}^{\mathrm{m}}$ possibility. No further findings were reported.

During the ${ }^{170} \mathrm{Er}$ beam experiments, the $A=171$ mass chain was studied to obtain more decay data and try to assemble a desay scheme. Nanural erbium and ${ }^{170}$ Er enriched ion sources were used by the SuperHILAC to deliver beam intensities of 30 to 82 particle nanoamperes on target for -45 hours. A tape cycle time of $128 \mathrm{~s}$ was used this time since the $49 \mathrm{~s}$ half-life was known. The production rate of ${ }^{171}$ Ho was much greater during this experiment, due to much larger beam intensities and probably due to the more favorable three nucleon transfer reaction $((2 n)$ in, (1p) out) relative to the five nucleon transfer reaction $((3 p, 2 n)$ out) for the ${ }^{176} \mathrm{Yb}$ experiment. However, other $A=171$ isotopes, mainly ${ }^{17} I_{\mathrm{Er}}$ and ${ }^{17} 1_{\mathrm{L} u}$, were 
Table 5.6. Summary of $A=171$ isobar information [Shi84] for nuclei that can be sepurated in OASIS.

\begin{tabular}{|c|c|c|c|}
\hline Isotope & Half-life & Decay Mode & Q value (MeVa \\
\hline${ }^{171} \mathrm{Hf}$ & $12.1 \mathrm{~h}$ & EC, $\beta^{+}$ & $2.4(2)$ \\
$171 \mathrm{Lu}$ & $8.24 \mathrm{~d}, 1.32 \mathrm{~m}^{\mathrm{b}}$ & $\mathrm{EC}, \beta^{+}, \mathrm{IT}$ & $1.480(2)$ \\
$171 \mathrm{Yb}$ & stable & -- & - \\
$171 \mathrm{Tm}$ & $1.92 \mathrm{y}$ & $\beta^{-}$ & $0.0964(10)$ \\
${ }^{171} \mathrm{Er}$ & $7.52 \mathrm{~h}$ & $\beta^{-}$ & $1.4907(13)$ \\
${ }^{171} \mathrm{Ho}$ & $72.4 \mathrm{~s}, 83.0 \mathrm{~s}^{\mathrm{c}}$ & $\beta$ & $3.16^{\mathrm{d}}$ \\
\hline
\end{tabular}

a $Q$ value reported is for ground state decay.

b 171 Lu has an IT decaying isomer at $71.1 \mathrm{keV}$. Ground state halflife is listed first.

c Listed $\mathrm{T}_{1 / 2}$ values are predictions, [Kla84] and [Tak73] respectively.

d $Q$ value is an average from rables compiled in [Hau88].

made with even larger cross sections than ${ }^{171} \mathrm{Ho}$. A summary of the half-lives, decay modes, and $Q$ values of the $A=171$ isobars [Shi84] that could have been produced in the experiment are listed in Table 5.6. The $Q_{45}$ - value for ${ }^{171} \mathrm{Ho}$ is an average from the mass compilation of Haustein [Hau88]. The maximum $\beta$ energy obtainable for the other $A=171$ isotopes results from ${ }^{171}$ Er decay with $2.3 \%$ of its $\beta$ intensity feeding the ground state or the $5.03-\mathrm{keV}$ level in ${ }^{171} \mathrm{Tm}$. The main $\beta$ branch (94\%) of ${ }^{171} \mathrm{Er}$ feeds a level at $425 \mathrm{keV}$ in ${ }^{171} \mathrm{Tm}$ giving a maximum beta energy of 1.065(94) MeV [Shi84].

The beta spectrum measured in the ${ }^{170}$ Er experiment for the $A=171$ mass chain is shown in Figure 5.19a. The high-energy $\beta$ - particles, 1.0 to $3.0 \mathrm{MeV}$, were seen to decay with a half-life of $56(4) s$ after background suberaction (see inset 


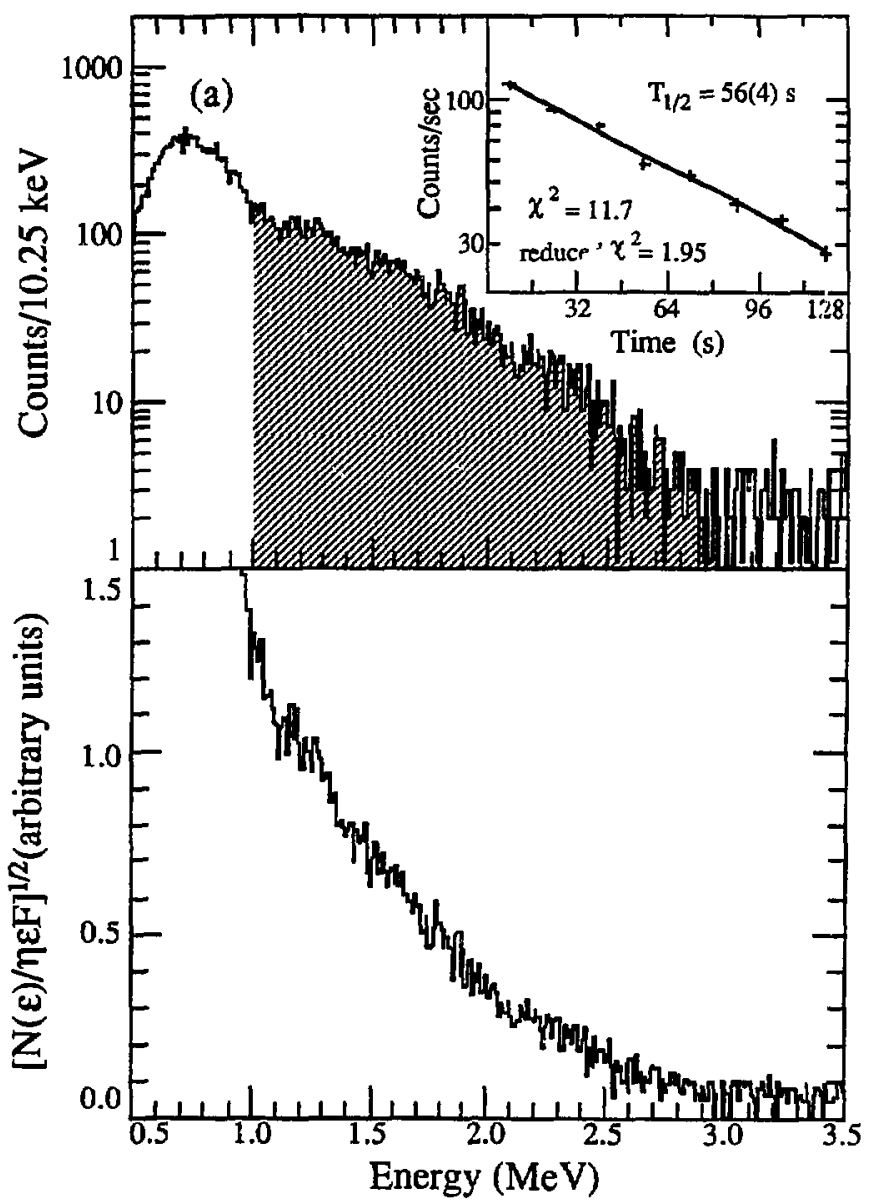

Figure 5.19. Beta decay data for $A=171$ mass chain; (a) beta particle spectrum measured in the beta telescope and (b) Fermi-Kurie plot. The inset in (a) shows the decay of 1.0 to $3.0 \mathrm{MeV}$ beta particles (shaded portion of spectrum), after subtraction of background. 


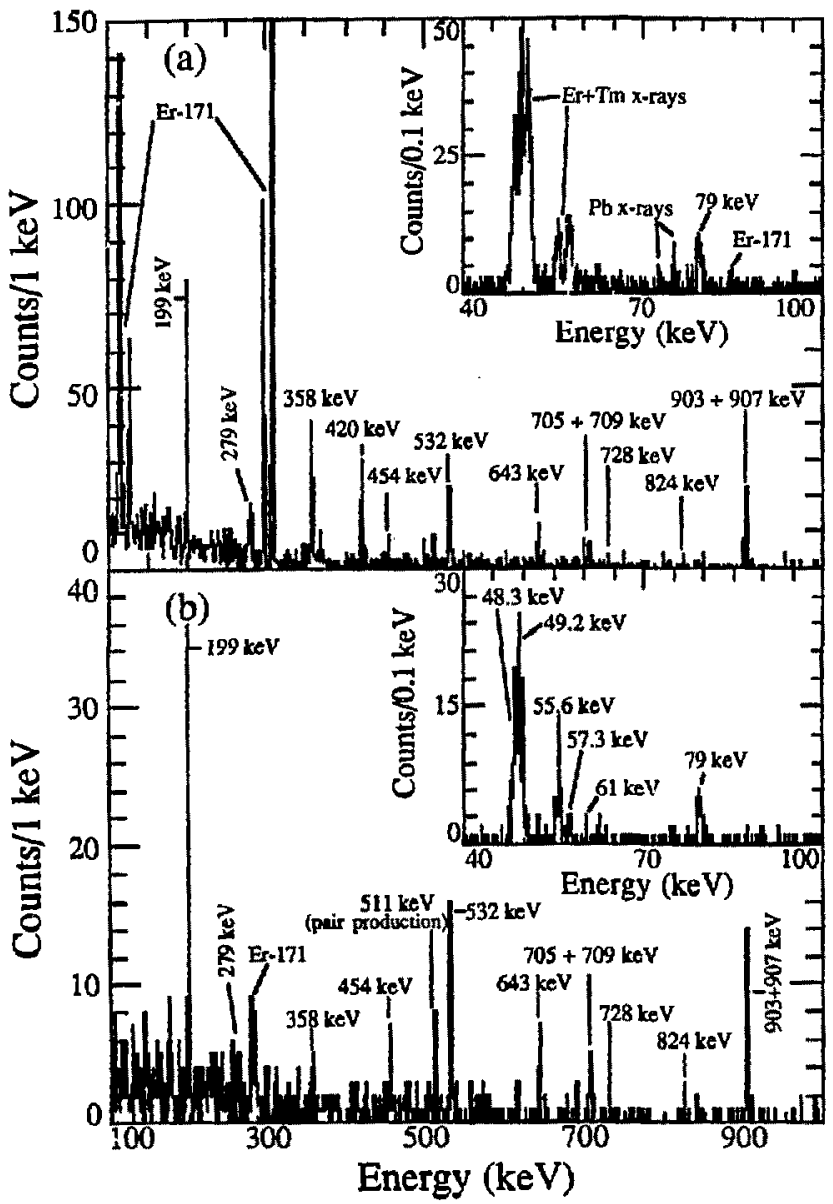

Figure 5.20. Gamma spectra in coincidence with beta particles measured in the beta telescope for the $A=171$ mass chain; (a) for betas with $E \geq 0.3 \mathrm{MeV}$ and (b) for betas with $E \geq 1.0 \mathrm{MeV}$. The insers show the $x$-ray region for each beta gatt. 
to Figure 5.19a). A Fermi-Kurie analysis was performed on this beta data, but it did not show a single $\beta$-component in the high-energy region, see Figure $5.19 \mathrm{~b}$. Spectra of the $\gamma$ rays measured in the beta telescope in coincidence with beta particles with energies $20.3 \mathrm{MeV}$ are shown in Figure 5.20a and its inset. The labeled $\gamma$ lines were previously unobserved. Contamination from ${ }^{171} \mathrm{Er} \gamma$ rays at $-120 \mathrm{keV}, \sim 300$ $\mathrm{keV}$ and $\operatorname{Tm} \mathrm{K} \mathrm{x}$-ray energies is indicated. Looking at $\gamma$ rays coincident with $\beta$ particles with energies $21.0 \mathrm{MeV}$ produced mivin cleaner spectra as shown in Figure $5.20 \mathrm{~b}$ and its inset. This gate removed all of the $\operatorname{Tm} \mathrm{K} \times$ rays and left only a small contamination from the strongest ( $65.6 \%$ absolute intensity) $\gamma$ transition of ${ }^{171} \mathrm{Er}$ decay at $308 \mathrm{keV}$ [Gra72].

The $\gamma$ rays seen in coincidence with the high-energy beta particles (Figure 5.20b) were analyzed in the singles data. Difficulties arose for the analysis of the 199- and 279- $\mathrm{keV} \gamma$ rays due to interfering radiations at these energies induced in the detectors by the neutron background. Analysis of the 358-keV $\gamma$-ray was complicated by a sum peak from the $308-k e V \gamma$ ray and the $T m K_{\alpha} x$ rays in ${ }^{171} \mathrm{Er}$ decay [Gra72]. The 61-keV $\gamma$-ray could not be identified in the singles data due to Lu $K_{\beta} \times$ rays at this energy. These $\gamma$ rays were all short-lived with an errorweighted average half-life of 54(3) s. Table 5.7 lists the Er $\mathrm{K} \times$ rays and fifteen $\mathrm{y}$ rays assigned to the decay of ${ }^{171}$ Ho with their measured relative intensities and $\gamma$-ray coincidences. The $61.2-\mathrm{keV} \gamma$-ray intensity was determined relative to the $79-\mathrm{keV} \gamma$ ray in a $\gamma$ spectrum measured in coincidence with beta signals in the plastic scintillator. The $1687-\mathrm{keV} \gamma$ ray was first noted in the coincidence spectra with $x$ rays and the $79-\mathrm{keV} \gamma$ ray and was later seen in coincidence with $\beta$ - particles. Six of the $\gamma$ rays listed in Table $5.7(79,199,279,358,705$, and 903 keV) were observed previously in the adopted level scheme for ${ }^{171} \mathrm{Er}$ [Shi84]. 
Table 5.7. Gamma-ray energies, $E_{\gamma}$, intensities, $I_{\gamma}($ rel.), and $\gamma$ coincidences measured in the decay of ${ }^{171} \mathrm{Ho}$.

\begin{tabular}{|c|c|c|}
\hline$E_{\gamma}(\mathrm{keV})$ & $I_{y}(\mathbf{r e l})^{\mathrm{a}}$ & Ccincident $\psi$-rays $(\mathrm{X}=\mathrm{Er} \mathrm{K} \times$ rays) \\
\hline $48.2 \mathrm{Er} \mathrm{K}_{\mathrm{O} 2}$ & $51(8)$ & $\begin{array}{l}X,(61), 199,279,(454),(642), \\
(708),(903),(907),(1687)\end{array}$ \\
\hline $49.1 \mathrm{Er} \mathrm{K}_{\alpha 1}$ & $106(7)$ & b \\
\hline $55.7 \mathrm{Er} \mathrm{K}_{\hat{\rho 1}}$ & $34(3)$ & $\mathrm{X}, 199,279,(358),(454),(824)$ \\
\hline $61.2(5)$ & 4(2) & $\mathrm{X}, 199,642$ \\
\hline $79.1(1)$ & $25(2)$ & $x, 279,(454),(824),(1687)$ \\
\hline $198.6(1)$ & $88(23)$ & $x, 61,642,705,(708)$ \\
\hline $279.2(4)$ & $60(9)$ & $x, 79$ \\
\hline $358.3(3)$ & $49(33)$ & $x$ \\
\hline $453.8(3)$ & $13(4)$ & $x, 79$ \\
\hline $532.2(1)$ & $58(4)$ & $\mathbf{x}$ \\
\hline $642.5(2)$ & $39(5)$ & $X,(61), 199$ \\
\hline $704.7(3)$ & $27(5)$ & $x, 199$ \\
\hline $708.5(1)$ & $34(19)$ & $\mathrm{X}, 199$ \\
\hline $727.6(1)$ & $44(5)$ & $\mathbf{x}$ \\
\hline $823.9(5)$ & $30(6)$ & $\mathrm{X}, 79$ \\
\hline $903.3(4)$ & $100(6)$ & c \\
\hline $907.2(2)$ & $57(6)$ & $(\mathrm{X})$ \\
\hline $1687.1(10)$ & $25(8)$ & $\mathrm{x},(79)$ \\
\hline
\end{tabular}

a Intensities are relative to 100 for the $903.3-\mathrm{keV}$ gamma transition.

b Clean coincidence gate could not be set due to interferences of Tm $K_{\mathbf{\alpha} 2} \times$ rays.

c No coincidences were observed. 
The proposed decay scheme for ${ }^{17}{ }_{1} \mathrm{Ho}$, based on the $\gamma$-ray coincidence and intensity information and the adopted level scheme [Shi84], is shown in Figure 5.21. This decay scheme is consistent with decay via many beta groups and the observation of a multi-component high-energy beta spectrum in Figure 5.19b. A half-life of 200(30) ns, Figure 5.22, was measured for the 199-keV level, in good agreeme.it with previously published results of $210(10) \mathrm{ns}$ [Shi84]. The placement of the ievel at $841-\mathrm{keV}$ is uncertain because of the possible reversal of the order of the 61 - and $641-\mathrm{keV} \gamma$-cascade and could alkematively be placed at $259.8 \mathrm{keV}$. Thare is no indication of direct $\beta$ - feeding to this level. The beta intensities and log $f$ 's listed in Figure 5.21 are based on no ground-state feeding. Since $\beta$ transitions have been measured between the 7/2-[523] proton and the 5/2-[512] neutron states [Led78], ground-state feeding is expected in ${ }^{171}$ Ho decay, but could no: be determined. The spin and parity assiguments for the ${ }^{171} \mathrm{Er}$ levels in the decay scheme are from the adopted level scheme [Shi84]. The assignment of the ${ }^{171} \mathrm{Ho}$ parent state (the $67^{\text {th }}$ proton) as 7/2-[523] was discussed in section 5.1b. The upper-limit, assuming no ground-state feeding, for beta feeding of the 199-keV level is $\leq 2 \%$, but because decay to this level would be highly forbidden $(\Delta I=3, \Delta \pi=n o)$, no beta feeding to this level is proposed. A ..alf-life of $55(3) \mathrm{s}$ was determined as a weighted average from all the available gamma and heta decay information.

The beta spectrum measured in coincidence uith 199-, 643-, 705-, 709-, 824-, 903-, and $907-\mathrm{keV} \gamma$ rays, and hence feeding the 903- and 907-keV levels, is shown in Figure 5.23a. The Fermi-Kurie plot of this spectra is shown in Figure $5.23 \mathrm{~b}$ with an endpoint of $2.3(6) \mathrm{MeV}$. This is consistent with the predicted average $\mathrm{Q}_{\beta}$ - value of $3.2 \mathrm{MeV}$ [Hau88]. A Q $\beta$. value of 3.2(6) MeV is assigned to this isotope. 


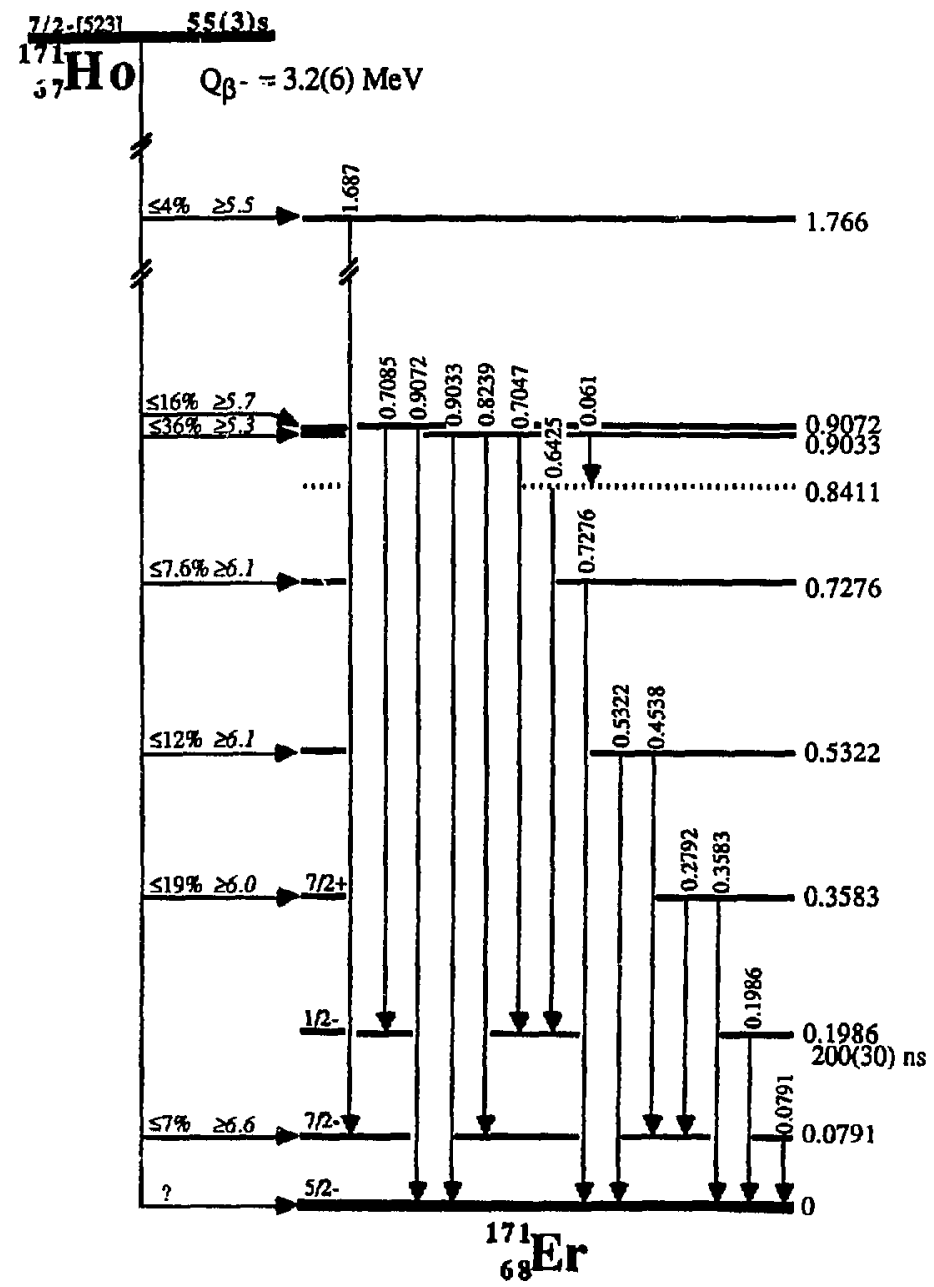

Figure 5.21. Proposed decay scheme for 55-s Ho-171. All energies are in MeV. Beta branches and $\log f t$ 's ane based on no ground-state beia branch. 


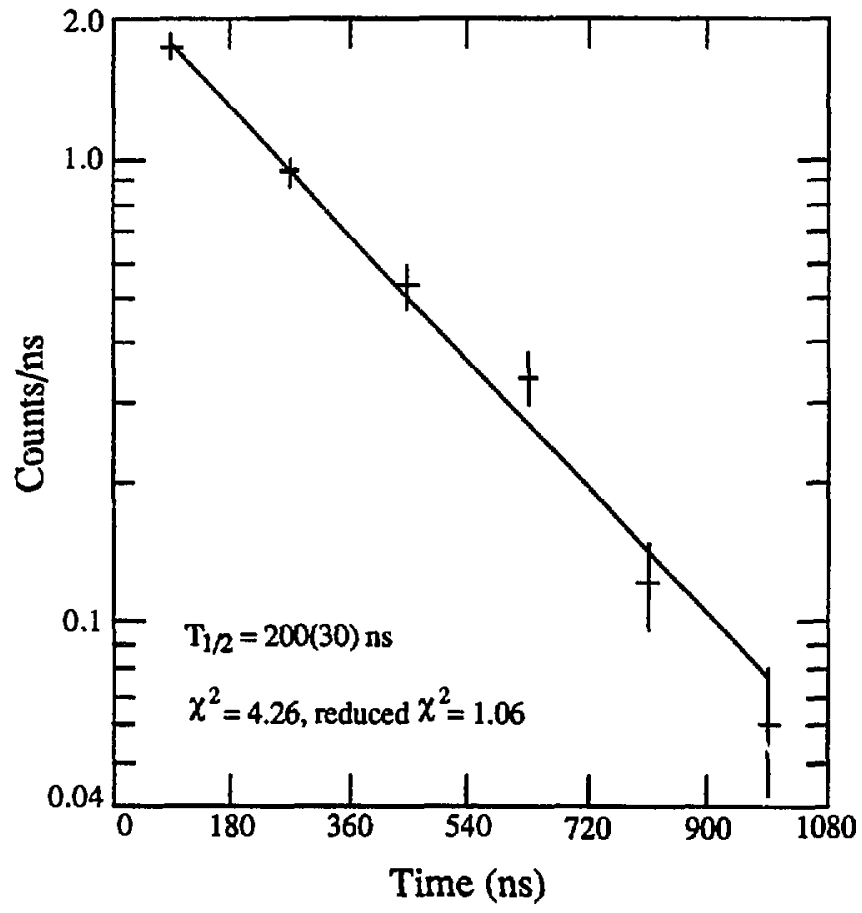

Figure 5.22. Single-component decay fit to the timing information between $199-\mathrm{keV}$ gamma rays and radiations feeding the 199-keV level in Er- 171 . 


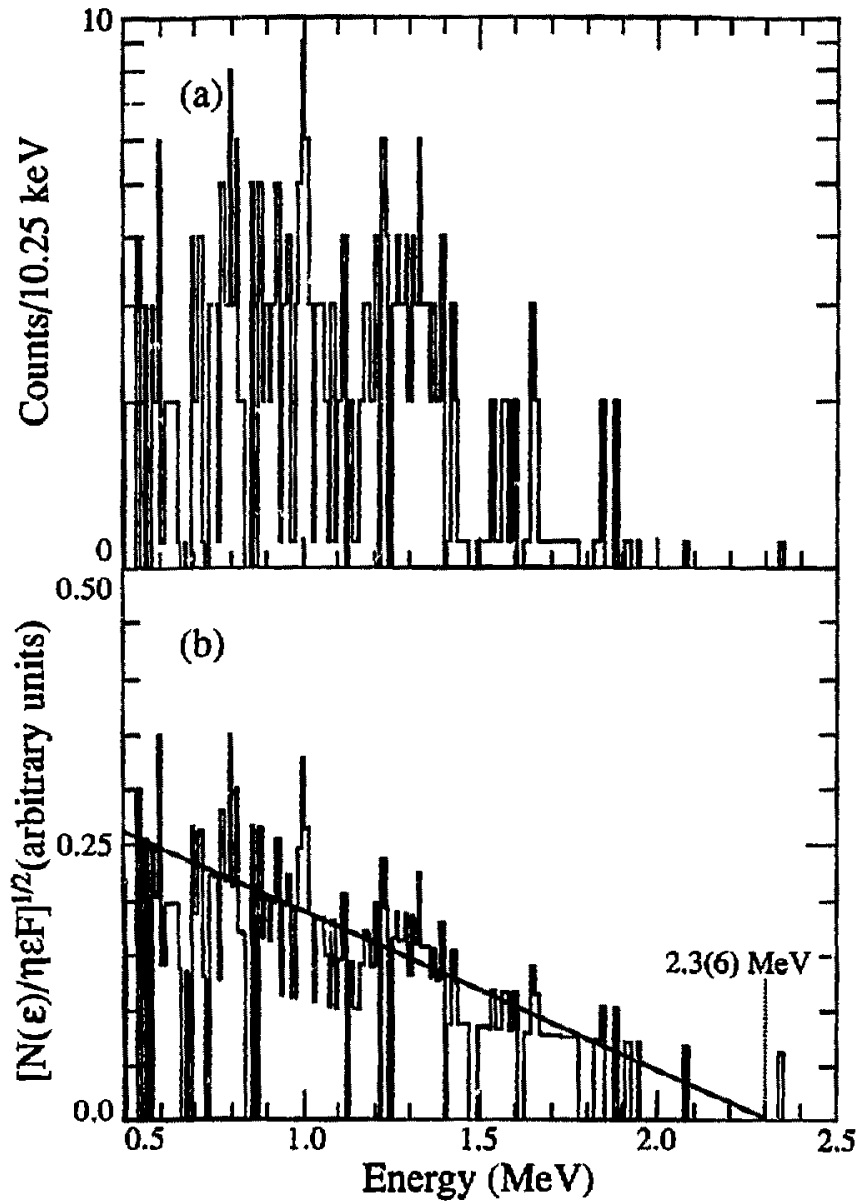

Figure 5.23. Beta decay data measured in coincidence with $199,642,705,708$, 824,903 , and $907 \mathrm{keV}$ gamma rays (gamma transitions associated o.., th beta dutay to the proposed 903 and $907 \mathrm{keV}$ levels of $\mathrm{Er}-171$ ) of Ho-171 decay; (a) beta particle spectrurn and (b) Fenmi-Kurie plot with a 2.3(6) MeV endpoint. 


\section{4. ${ }^{174} \mathrm{Er}$ - NEW ISOTOPE}

The $A=174$ mass chain was the first multinucleon transfer reaction experiment performed witt. OASIS. Event descriptions and parameters were not optimum for $\beta$-decaying isotopes for the half-lives in this region. The lessons learned from this ${ }^{176} \mathrm{Yb}$ beam experiment gave information which allowed the design of the successful ${ }^{170} \mathrm{Er}$ beam experiment in which almost all of the results mentioned in the previous sections were obtained in only two weeks of beam time. Even though the detection system was not optimized for neutron-rich nuclides in this experiment, useful new results were obtained for the $A=174$ mass chain. Natural ytterbium ion sources were used in the SuperHILAC to deliver beam intensities of 5 to 24 particle nanoarnperes of ${ }^{176} \mathrm{Yb}$ ions on target in OASIS for $\sim 60$ hours. The experiment was performed to search for the new isotope ${ }^{174} \mathrm{Er}$. Mass predictions compiled by Haustein [Hau88] gave an average $\mathrm{Q}_{\beta}$ - value of $1.7 \mathrm{MeV}$ (the value given by Comay et al. [Com88] was excluded because it was crastically different from the other eight values listed for this isotope). The half-life predictions were $80 \mathrm{~s}$ [Kla84] and $15 \mathrm{~m}$ [Tak73]. A half-life of $395 \mathrm{~s}$ was calculated using the GTBD code [Tak88] and the $1.7 \mathrm{MeV} \mathrm{Q}_{\beta}$ - value above. A tape cycle time of $512 \mathrm{~s}$ was chosen initially, and when $\mathrm{Tm} \mathrm{K}$ x rays (from the decay of either ${ }^{174} \mathrm{Er}$ or a ${ }^{174} \mathrm{Tm}$ isomser) were seen to decay with $\sim 175 \mathrm{~s}$ half-life on-line, the tape cycle time was continued. The information on ${ }^{174} \mathrm{Er}$ decay contained below has been previously published [Cha89] unless otherwise noted.

The twelve gamma rays and three $\operatorname{Tm} \mathrm{K} \times$ rays with energies, relative intensicies, and gamma coincidences listed in Table 5.8 are assigned to the decay of the isotope ${ }^{174} \mathrm{Er}$. These gamma rays decayed with a half-life of $3.3(2) \mathrm{m}$ which did 
Table 5.8. Gamma-ray energies, $E_{\gamma}$ intensities, I (rirel.), and coincidences assigned to the decay of $3.3 \mathrm{~m}^{174} \mathrm{Er}$.

\begin{tabular}{|c|c|c|}
\hline $\mathrm{E}_{\gamma}(\mathrm{keV})^{\mathbf{a}}$ & $I_{\gamma}(r e l .)^{b}$ & Gamma Coincidences $c$ \\
\hline 49.7 $\operatorname{TmK}_{\alpha 2}$ & $137(5)$ & $\begin{array}{l}X,(58.5), 71.7,100,122, \\
(130), 152,637,642\end{array}$ \\
\hline $50.7 \mathrm{Tm} \mathrm{K}_{\alpha \mathbf{l}}$ & 263(9) & $d$ \\
\hline $57.4 \mathrm{Tm} \mathrm{K}_{\beta 1}$ & $67(3)$ & $\begin{array}{l}x, 71.7,100,(122), 152 \\
(637),(642)\end{array}$ \\
\hline $58.5 e$ & & \\
\hline 71.7 & $15(1)$ & $X,(58.5),(122), 637,643$ \\
\hline 100.4 & $100(1)$ & $\mathrm{X}, 152$ \\
\hline 121.8 & $39(6)$ & $x,(58.5),(71.7), 130$ \\
\hline 130.4 & $42(8)$ & $122,637,643$ \\
\hline 151.8 & $91(15)$ & $\mathrm{X}, 100.4$ \\
\hline 636.7 & $27(5)$ & $X,(58.5),(71.7), 130$ \\
\hline 642.7 & $25(2)$ & $x,(58.5),(71.7), 130$ \\
\hline 708.4 & $93(7)$ & $(58.5)$ \\
\hline 714.4 & $56(6)$ & $(58.5)$ \\
\hline 766.9 & 92(9) & f \\
\hline 772.9 & $75(8)$ & $f$ \\
\hline
\end{tabular}

a The uncertainty in the $\gamma$-ray energies is $\pm 0.2 \mathrm{keV}$.

b Intensities are relative to 100 for the $100.4-k e V \gamma$ transition.

c $X=\operatorname{Tm} K x$ rays. () denotes a weak coincidence.

d Clean coincidence gate could not be set.

e Not seen in the singles data. See text.

$f$ No coincidences measured. 
not match any of the known half-lives of this isobaric chain. All of the $\gamma$ rays were seen in coincidence with $\beta$-particles in the $\beta$ telescope and plastic scintillator and were not in coincidence with any of the known $\gamma$ rays from the decay of ${ }^{174} \mathrm{Tm}$ [Bro84]. Some of the gamma rays were seen in coincidence with $T m \mathrm{~K} x$ rays as indicated in Table 5.8. The first and last points (new half-life and $T \mathrm{~m} \mathrm{~K} \mathrm{x}$-ray coincidences) are either consistent with a possible isomer of ${ }^{174} \mathrm{Tm}$ or the new isotope ${ }^{174} \mathrm{E}$, however the ${ }^{174} \mathrm{Tm}$ isomer is ruled out by points two and three ( $\beta$ cuincidences and absence of ${ }^{174} \mathrm{Tm}$ y coincidences). The 58.5-keV gamma transition was nct seen in the HPGe singles data due to interfering $x$-ray activity (Yb $K_{\beta} \times$ rays from ${ }^{174} \mathrm{Tm}$ decay and $\mathrm{Tm} \mathrm{K}_{\beta} \times$ rays from the decay of ${ }^{174} \mathrm{Er}$ itself!) at that energy, but was clearly identified in the coincidence spectra. The low energy part of the HPGe detector singles spectrum is shown in Figure 5.24 with all pertinent peaks labelled.

Based on the coincidence and intensity information, the decay scheme in Figure 5.25 has been proposed for ${ }^{174} \mathrm{Er}$. The even-even isotope, ${ }^{174} \mathrm{Er}$ has a $0+$ ground state. The two strong $\beta$ - transitions to the 766.9- and 772.9-keV levels are similar to the $0+$ to $1+$ and (1)- $\beta$ - transitions in $172 \mathrm{Er}$ decay [Led78]. Assuming then a spin difference of one unit between the ${ }^{174} \mathrm{Er}$ ground state and the $770-\mathrm{keV}$ levels, the observed strong $\gamma$ transitions to the proposed ${ }^{174} \mathrm{Tm}$ ground state are inconsistent with a tentative 4-assignment for the ${ }^{174} \mathrm{Tm}$ ground state [Bro84]. This discrepancy can be explained by an isomer in either ${ }^{174} \mathrm{Tm}$ or ${ }^{174} \mathrm{Er}$ which would change the spins of the parent or daughter ground states. Even-even isotopes, such as ${ }^{174} \mathrm{Er}$, rarely have isomers, but for $\mathrm{N}=106$ isolopes, isomers of even-even ${ }^{176} \mathrm{Yb}$ and $178 \mathrm{Hf}$ do exist. However, their isomers both decay by intense isomeric transitions [Led78]. Isomers are highly probable for the odd-odd isotope 


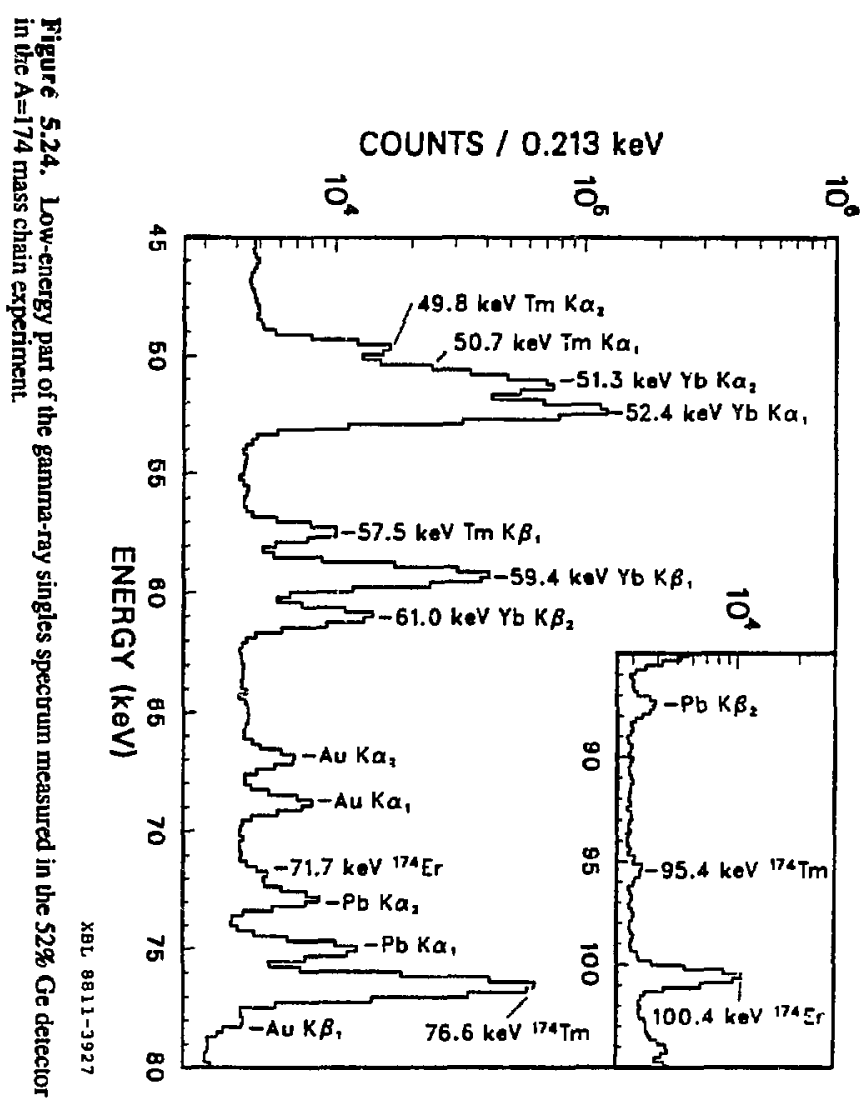




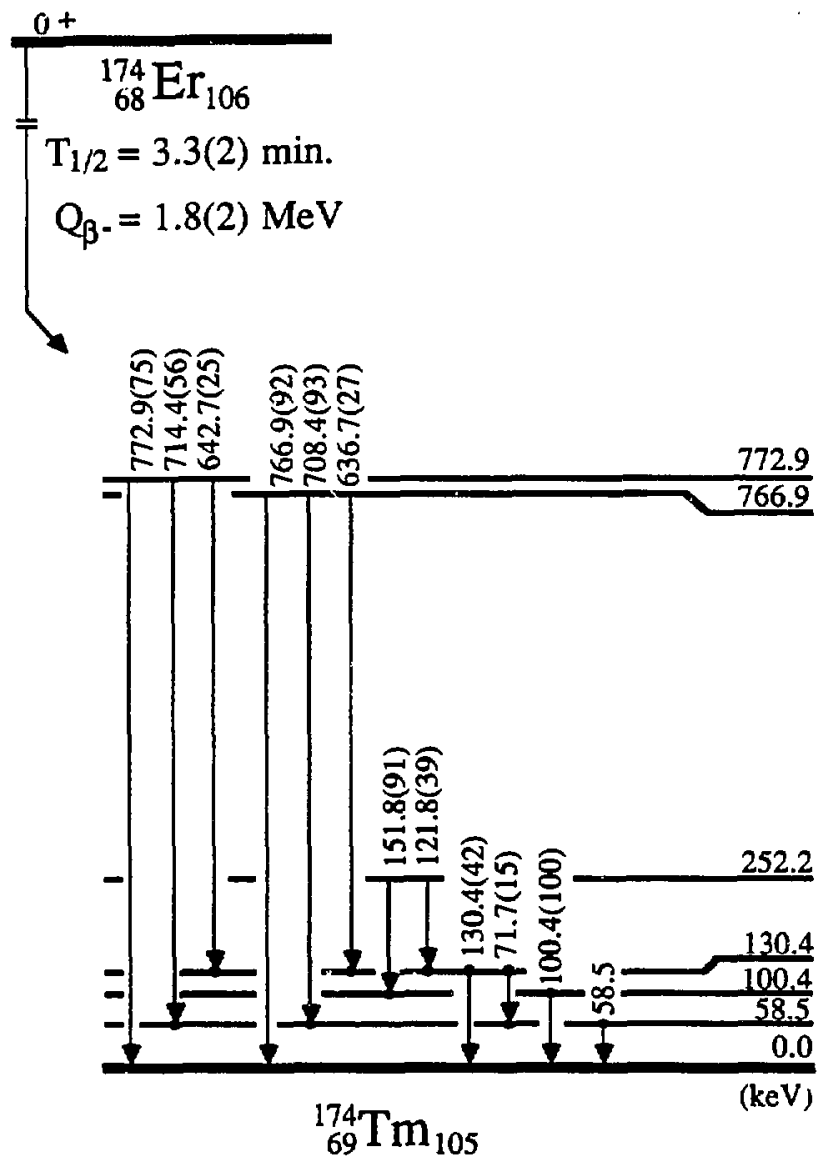

Figure 5.25. Proposed decay scheme for Er-174. Intensities are relative to the 100.4keV transition. 
${ }^{174} \mathrm{Tm}$. Consequently, spin and parities of the ${ }^{174} \mathrm{Tm}$ levels in Figure 5.25 have not been proposed.

After the $\beta$ - spectrum analysis programs were written and used with excellent success on the ${ }^{170} \mathrm{Er}$ beam experiment data, the beta data for the $\mathrm{A}=174$ mass chain was reanalyzed using similar techniques. The $\beta^{-}$spectrum feeding the 767- and 773-keV levels in ${ }^{174} \mathrm{Tm}$, shown in Figure 5.26a, was generated by gating with the $637,643,708,714,767$, and $773 \mathrm{keV}$ gamma rays. The Fermi-Kurie plot and endpoint of $1.05(23) \mathrm{MeV}$ are shown in Figure 5.26b. The $\mathrm{Q}_{\beta}$ - value of 1.8(2) $\mathrm{MeV}$ is suggested for the decay of 3.3-m $174 \mathrm{Er}$, in good agreement with average predicied value of $1.7 \mathrm{MeV}$. The $\beta$ telescope calibration for the $176 \mathrm{Yb}$ experiment was checked by measuring the $\beta$ - particle spectrum in coincidence with $366-\mathrm{keV} \gamma$ rays from the decay of ${ }^{174} \mathrm{Tm}$, shown in Figure 5.27a. A $\beta$ - endpoint of $1.22(5)$ $\mathrm{MeV}$ was obtained from the Fermi-Kurie plot in Figure 5.27b, in good agreement with the value of $1.21(5) \mathrm{MeV}$ reported in the literature [Bro84]. The $\beta$ - endpoint results reported here were not published in the ${ }^{174} \mathrm{Er}$ discovery paper [Cha89].

\subsection{TARGETION SOURCE TESTS}

The nomal targevion source geometry of OASIS was designed to optimize production and ionization of compound nucleus recoiling products. The target was placed immediately upstream of narrow capillary tubes with small acceptance angles to selectively allow the compound nucleus recoils (small lab frame angle) to pass and discriminate against other taansfer reaction products. (See Figure 3.4) For the multinucleon transfer reactions snudied in this research. large recoil angles were expected (see discussion in chapter 2). During the first ${ }^{176} \mathrm{Yb}$ beam experiment, modifications to the anode of the ion source were made as shown in Figure 5.28a. 


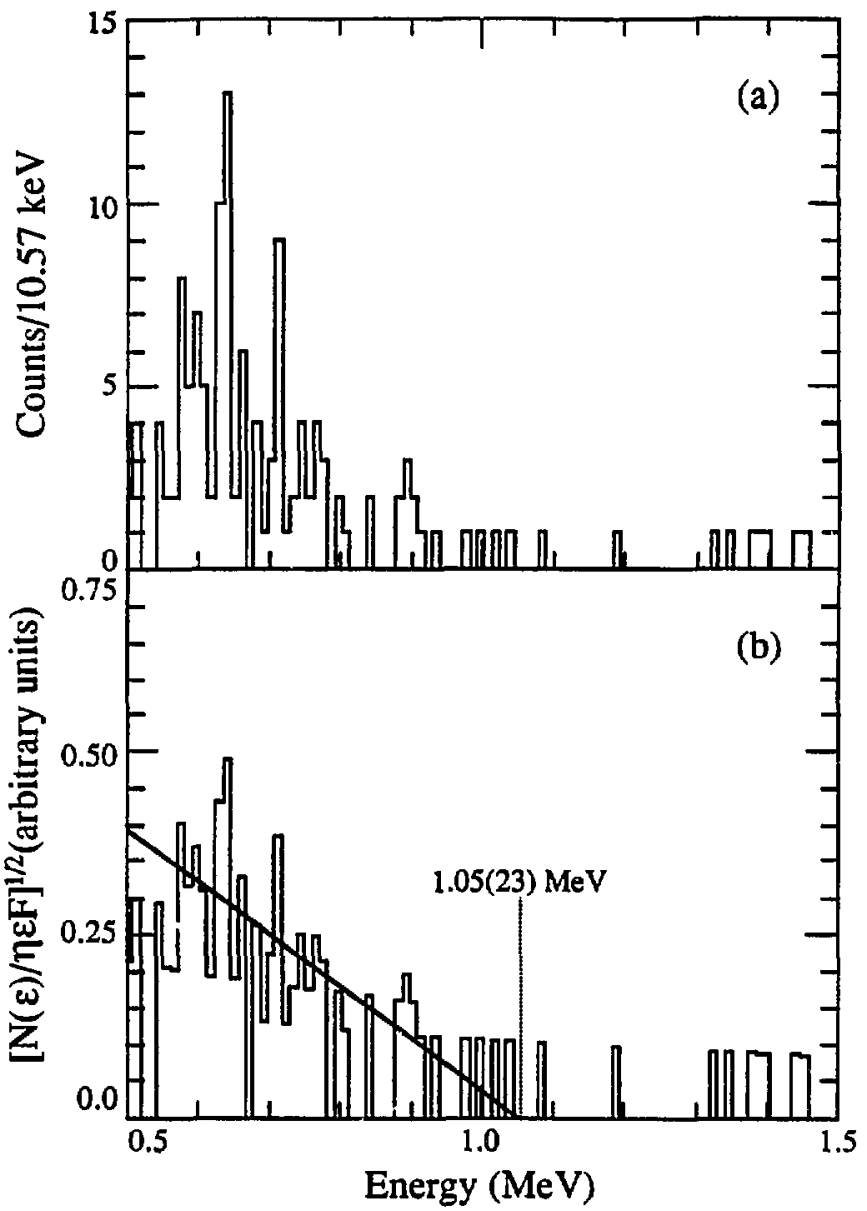

Figure 5.26. Beta particle data measured in coincidence with $637,643,708,714$, 767 , and $773 \mathrm{keV}$ gamma rays in the $A=174$ mass chain; (a) beta particle spectrum and (b) Fermi-Kurie plot and 1.05(23) MeV endpoint. 


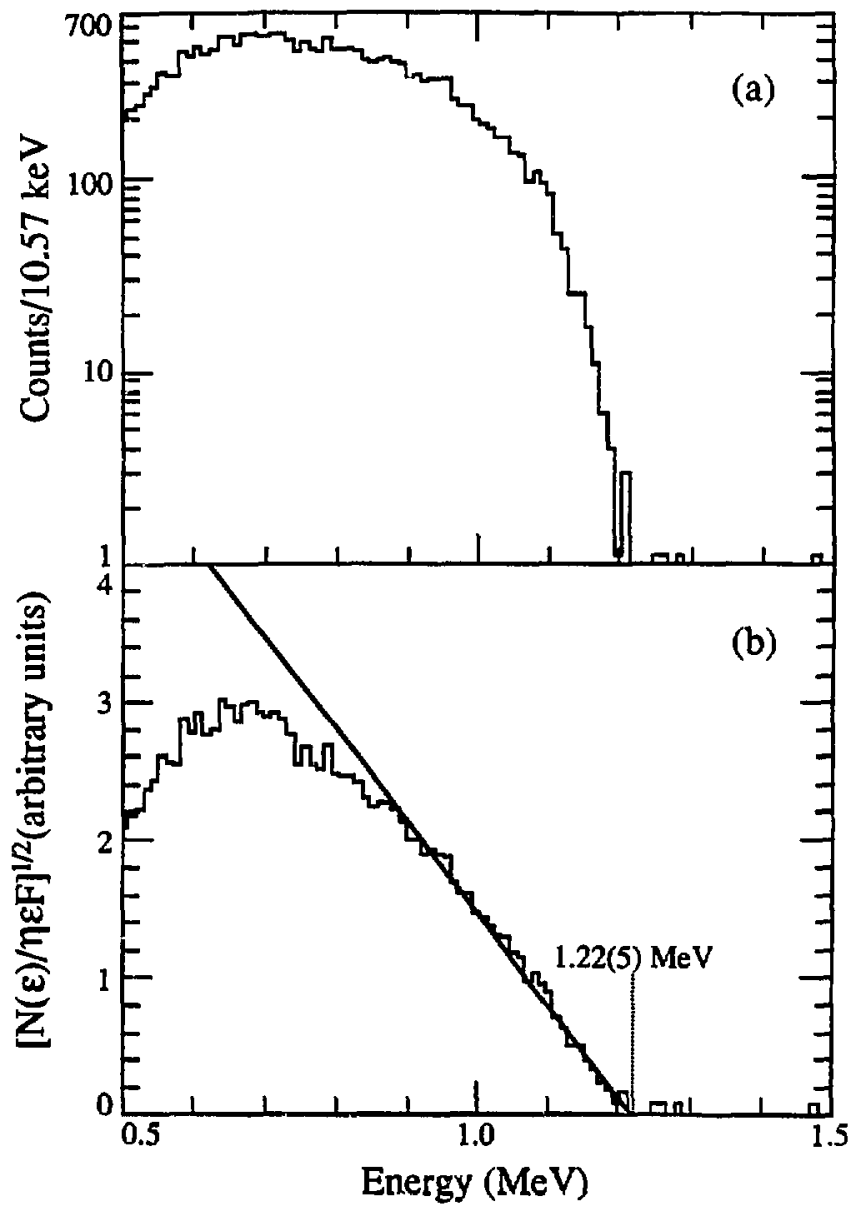

Figure 5.27. Beta particle data measured in coincidence with 366-keV gamma rays from the decay of $\mathrm{Tm}-174$; (a) beta particle spectrum measured in the beta telescope and (b) Fermi-Kurie plot and beta endpoint of $1.22(5) \mathrm{MeV}$. 


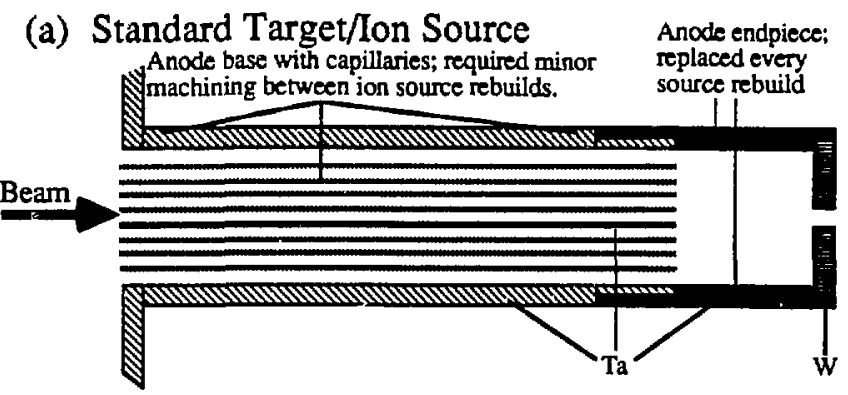

(b) W coated Ta (W/Ta) Target/Ion Source

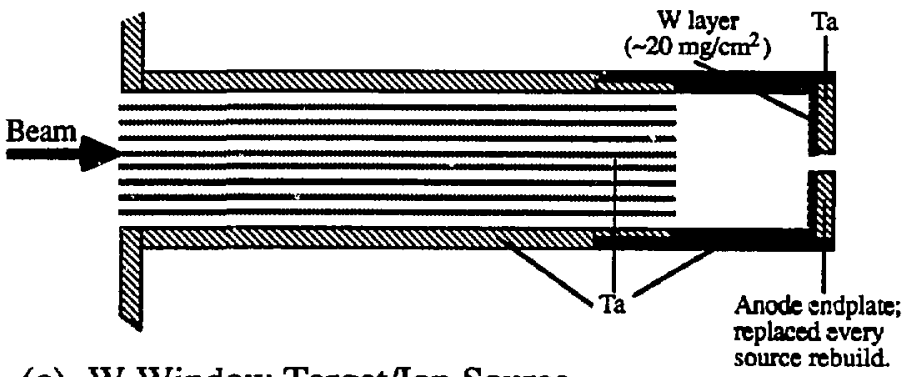

(c) W Window Target/Ion Source

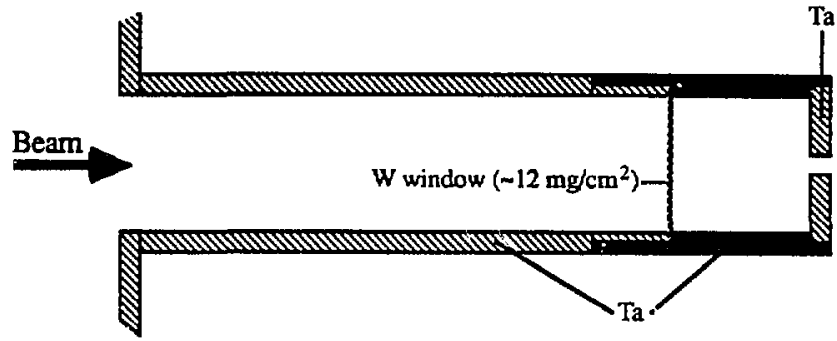

Figure 5.28. Targetion source geometries used in the Er-170 beam experiments; (a) standard targevion source, (b) W/Ta target/ion source, and (c) W windcw target/ion source. 
The anode (anode base, capillaries, anode endpiece and endplate) was normally made entirely of Ta because of its high temperature stability and good diffusion and surface ionization properties. For this research, the target was removed and the $\mathrm{Ta}$ endpiece was replaced with W. This endpiece served as the target, recoil catcher, and to some degree as ionizer. Since the target was removed, the ion source was open to the SuperHILAC vacuum. Only the capillaries stopped the recoils, once in a gaseous state, from migrating back into the vacuum of the SuperHILAC. During the ${ }^{176} \mathrm{Yb}$ beam experiment, one other target/ion source geomerry was tried; where a Ta endpiece was used and $a \sim 17 \mathrm{mg} / \mathrm{cm}^{2} \mathrm{~W}$ foil was placed just downstream of the capillaries. This foil was thicker than the calculated $\sim 11.5 \mathrm{mg} / \mathrm{cm}^{2}$ of W necessary to slow the ${ }^{176} \mathrm{Yb}$ beam below the Coulomb barrier. This source showed almost a two-fold increase in the production rate, but no quantitative results were obtained.

During the $170 \mathrm{E}$ r beam experiment attempts were made to quantify the differences in various target/ion source geometries. The three geometries tested are shown in Figure 5.28. The $\mathrm{W}$ coated (W/Ta) geomery (Figure 5.28b) was used since Ta shows better diffusion than $W$ and is much easier to machine. The $\sim 20$ $\mathrm{mg} / \mathrm{cm}^{2} \mathrm{~W}$ layer was acually evaporated onto both sides of the endplate and was thicker than the $-12.6 \mathrm{mg} / \mathrm{cm}^{2}$ necessary to slow the $170 \mathrm{Er}$ beam below the Coulomb barrier. The $W$ window geometry (Figure 5.28c) was a modification of the $W$ foil source used in the $176 \mathrm{Yb}$ beam experiment where the capillaries were removed to allow the full bean to hit the $W$ window. The $12-\mathrm{mg} / \mathrm{cm}^{2}$ thickness was chosen on the following criteria: maximize the amount of $W$ encountered by beam with energy greater than the Coulomb barrier, and yet keep it thin enough to allow the recoils to exit the window even at large multinucleon transfer reaction angles. A computer code was written to determine the optimum target thickness. The code 
assumed the recoils emerged at the quarter-point angle [Wil80] (an over-estimate for all of the recoils, but a good average), calculated the recoil energy for a binary reaction, and, using range and stopping power tables [Hub80], determined if the target-like recoil had enough energy to exit the foil. Using the above criteria, it was determined that the maximum effective target thickness occurred at the Coulomb barrier thickness, $12.6 \mathrm{mg} / \mathrm{cm}^{2}$, and that $\sim 99 \%$ of the recoils would leave the window/target. A $12-\mathrm{mg} / \mathrm{cm}^{2} \mathrm{~W}$ foil was used since this was the closest commercially available thickness.

The tests of the three target/ion source geometries were performed with the $A=168$ mass chain and measuring the production rate of detected ${ }^{168} \mathrm{Ho}$ and ${ }^{168} \mathrm{Lu}$ gamma radiations. For ${ }^{168} \mathrm{Ho}, \gamma$ transitions at 741,815 , and $821 \mathrm{keV}$ were used, and for ${ }^{168} \mathrm{Lu}$, gamma transitions at 511 (from positron annihilation), 896, and 979 $\mathrm{keV}$ were used. Peaks were integrated on-line using the $52 \%$ Ge singles data, background was subtracted, and the results were normalized by dividing by the integrated beam intensity. Tests ware performed for -6 hours on each targetion source geometry to obtain good average relative intensities (number of backgroundcorrected $y$ rays detected in a set time divided by the beam integral) for each $y$ ray. The results of the tests are listed in Tables 5.9 and 5.10 where the results are also normalized to the standand target/ion source geometry. The W/Ta targetion source showed $\sim 25 \%$ increase versus the standand geometry for the production of $168 \mathrm{Ho}$ and ${ }^{168} \mathrm{Lu}$, and the $\mathrm{W}$ window geometry had a $110 \%$ increase in the production of $168 \mathrm{Ho}$ and a $40 \%$ increase in the production of $16 \% \mathrm{Lu}$. The differences between the increases of the ${ }^{168} \mathrm{Ho}$ and ${ }^{168} \mathrm{Lu}$ production for the W window source is not understood, but might have something do with a different reaction to produce 
Table 5.9. Results of the target/ion source tests on the production of 168Ho. Errors are escimated as $\sim 10 \%$.

\begin{tabular}{|c|c|c|c|c|}
\hline \multirow{2}{*}{$\begin{array}{c}\text { Targetion } \\
\text { Source }\end{array}$} & \multicolumn{2}{|c|}{ Relative Intensities of ${ }^{168} \mathrm{Ho} \gamma$ Rays } & \multirow{2}{*}{$\begin{array}{c}\text { Ave. Normalized } \\
\text { to Standard }\end{array}$} \\
\cline { 2 - 5 } & $741 \mathrm{keV}$ & $815 \mathrm{keV}$ & $821 \mathrm{keV}$ & 1.0 \\
Standard & 3.5 & 1.64 & 3.4 & 1.3 \\
W/Ta & 4.5 & 2.2 & 4.3 & 2.1 \\
W Window & 7.2 & 3.4 & 7.0 & 2.0 \\
\hline
\end{tabular}

${ }^{168} \mathrm{Lu}$, a (3p) in, (5n) out reaction channel, versus the (pn) out reaction for ${ }^{168} \mathrm{Ho}$ produccion. The $W$ window geometry was clearly the best one to use, but lasted oniy -6 hours at which point the window melted due to beam heating. The W/Tz target ion source geometry was used the most after the tests due to its increase in yield over the standard source, durability (it lasted $\sim 24$ hours like the standard ion/target ssurce), and the case of machining Ta versus $W$ endplates.

Table 5.10. Results of target/ion source test on the production of ${ }^{168} \mathrm{Lu}$. Errors are extimated as $\sim 10 \%$.

\begin{tabular}{|c|c|c|c|c|}
\hline \multirow{2}{*}{$\begin{array}{c}\text { TargetIon } \\
\text { Source }\end{array}$} & \multicolumn{2}{|c|}{ Rel. Intensities of 168 Lu Radiacions } & Ave. Normalized \\
\cline { 2 - 4 } & $511 \mathrm{keV}$ & $896 \mathrm{keV}$ & $979 \mathrm{keV}$ & to Standand \\
\hline Standard & 1.21 & 0.76 & 0.95 & 1.0 \\
W/Ta & 1.45 & 0.93 & 1.1 & 1.2 \\
W Window & 1.6 & 1.1 & 1.3 & 1.4 \\
\hline
\end{tabular}




\section{CONCLUSIONS}

Neutron-rich rare-earth nuclides were prcduced using multinucleon transfer reactions betweer ${ }^{170} \mathrm{Er}$ and ${ }^{176} \mathrm{Yb}$ ion beams and natw targets. Two new isotopes and one new isomer were discovered in this study. Decay schemes were deremined for these three nuclides and also for two previously discovered isotopes. A brief summary of the results for these nuclides will be discussed in the following paragraphs.

The ${ }^{176} \mathrm{Yb}$ beam experiment produced the new isotope ${ }^{174} \mathrm{Er}$ [Cha89], which had a measured half-life of 3.3(2) min.. Fifteen $x$ rays and $\gamma$ rays (Table 5.8) were assigned to the decay of this isotop" and were placed in the tentative decay scheme shown in Figure 5.25. The strong beta branches to the 767- and 773-keV levels in ${ }^{174} \mathrm{Tm}$, which both have strong ground state $\gamma$ transitions, ae inconsistent with a $0+$ assignment fo- ${ }^{174} \mathrm{Er}$ and a 4 - assignment for the measured ${ }^{174} \mathrm{Trn}$ ground state. An isomer of the odd-odd ${ }^{174} \mathrm{Tm}$ could explain the measured results. Arrother possiole explanation would be an isomer of eyen-even ${ }^{174} \mathrm{Er}$ which has an expected $0+$ ground state. Though even-even isomers are rare for this region, even-even $\mathrm{N}=106$ isomers exist for both ${ }^{176} \mathrm{Yb}$ and ${ }^{178} \mathrm{Hf}$. These isomers seem in result from the unpairing of the neutrons in the last neutron level, $7 / 2-[514]$, and with one neutron remaining in that level and one in the next neutron level, $9 / 2+[524]$, resulting in the 8- assignments for both of the ${ }^{176} \mathrm{Yb}$ and ${ }^{178} \mathrm{Hf}$ isomers. However, an 8 assignment for ${ }^{174} \mathrm{Er}$ is inconsistent with the proposed decay scheme and the ${ }^{174} \mathrm{Tm}$ ground state assignment. For the same reasons, a $0+$ assignment for ${ }^{174} \mathrm{Er}$ is inconsistent. Also, the 8 - isomers of ${ }^{176} \mathrm{Yb}$ and ${ }^{178} \mathrm{Hf}$ both decay by suong $I T$ branches [Led78]. This research suggests that the measured ${ }^{174} \mathrm{Er}$ parent state has a 
0+ spin and parity assignment. Attempts to solve the problems of the parent and daughter spins would require source intensities at least an order of magnitude greater than produced in this study since no evidence of isomers were seen using the $512 \mathrm{~s}$ tape cycle time. The SuperHILAC could possibly give a factor of 2 to 5 times greater beam intensities with $176 \mathrm{Yb}$ enriched ion sources and an optimally running accelerator, but this would not be efficient enough. However, using the 2 to 5 times more intense $11.4 \mathrm{MeV} / \mathrm{u}{ }^{176} \mathrm{Yb}$ beams obtainable at the UNLAC accelerator at GSI, would allow use of twice the previous target thichress. Yields of $\sim 10$ times the source intensities of this study then could be obtained, which might give new information.

In the $\mathrm{A}=168$ mass chail, studied during the ${ }^{170} \mathrm{Er}$ beam experiment, the decay scheme of 8.8(3)-m ${ }^{168}$ Dy was determined, Figure 5.4. Gamma- and x-ray intensities were obtained for the five previously identified $y$ transitions [Geh82] plus two new $\gamma$ transitions and the two Ho $K_{\alpha} \times$ rays, Table 5.2. During the analysis of the ${ }^{168} \mathrm{Dy}$ data, a new isomer, ${ }^{168} \mathrm{Hom}$, was identified. The isomer had a measured half-life of 132(4) s from Ho $\mathrm{K} x$ rays, but the proposed $I T$ y transition was not measured due to its large invernal conversion coefficient. The proposed decay scheme for ${ }^{168} \mathrm{Hom}$, Figure $5.9 \mathrm{~b}$, is consistent with similar isomerism seen in ${ }^{172} \mathrm{Lu}$ [Va162], Figure 5.9a. Also during the data analysis for this mass chain, the $Q_{\beta}$ value for 3.0-m ${ }^{168} \mathrm{Hos}$ [Kaw73] was determined. Gamma-ray coincident beta spectra were generated and Kurie plots calculated to determine $\beta$ - endpoints to three ${ }^{168} \mathrm{Er}$ levels, $\mathrm{Fi}_{\delta}$ gures 5.10-5.12. A Q $\beta$ - value of $2.93(3) \mathrm{MeV}$ is proposed for ${ }^{168} \mathrm{Hog}$.

The new isotope, ${ }^{169} \mathrm{Dy}$, was identified during the ${ }^{170} \mathrm{Er}$ beam experiment. High-energy $\beta$ - particles, with a 3.2(3) MeV endpoint, were observed to decay with 
a 43(11) $s$ half-life and showed no coincidences above background. Only a 1578$\operatorname{keV} \gamma$ ray decayed with a short half-life of $35(11) \mathrm{s}$, while all other $\gamma$ rays decayed with longer half-lives. Absence of Ho $\mathrm{Kx}$ rays indicates that there was no internal conversion of $\gamma$ rays from Dy $\beta$ - decay. The assignment of these activities to the decay of ${ }^{169}$ Dy was based on the agreement of the experimental half-life, 39(8) $\mathrm{s}$, and $Q_{\beta}$ - value, 3.2(3) MeV, with theoretical half-life predictions, $38 \mathrm{~s}$ [Kla84] and 64 $\mathrm{s}$ [Tak73], and mass table calculations for decay energies, 3.2 MeV [Hau88]. The proposed decay scheme for ${ }^{169} \mathrm{Dy}$ is shown in Figure 5.18.

The $A=171$ mass chain was studied in both the ${ }^{176} \mathrm{Yb}$ and ${ }^{170} \mathrm{Er}$ beam experiments in hopes of determinirig the decay scheme for ${ }^{171} \mathrm{Ho}$ [Ryk84, Ryk88, Ryk89]. The ${ }^{176} \mathrm{Yb}$ beam experiment yielded only enough information to confirm the discovery [Ryk84, Ryk88]. During the $170 \mathrm{Er}$ beam experiment, $18 \mathrm{x}$ rays and $\gamma$ rays (see Table 5.7) were measured to decay with a 55(3) s half-life. A partial decay scheme, Figure 5.21, was devised for the decay of ${ }^{171^{\prime}}$ Ho. Beta particle decay data for this mass chain had high-energy $\beta$ - particles that decayed with the conect halflife. Kurie analysis of $\beta$ - particles coincident with $\gamma$ rays depopulating the $900-\mathrm{keV}$ levels yielded a $\beta^{-}$endpoint of 2.3 (6) $\mathrm{MeV}$ and a $\mathrm{Q}_{\beta}$ - value of $3.2(6) \mathrm{MeV}$.

The beta telescope used on OASIS, $718 \mu \mathrm{m}$ Si/HFGe, produced very useful beta spectra for determining decay energies. Comparisons of the measured $Q_{\beta}$ values with those calculated from nuclear mass tables has always been a goal of this research. The most recent nuclear masses calculations have been compiled by Haustein [Hau88] and made available on magneric tape for ease of computer usage. Throughout this thesis, an average of the $Q_{\beta}$. values calculated using all the mass tables was used. An average was chosen over using a particular mass formula for the following reasons: the region studied was close to $\beta$ stability where most 
formulae should predict masses well, and selecting a formula by its fit to known masses in this region would be biased towards formulae that used a large number of known masses in determining ther-calculation paramerers. It was also believed by the author of this thesis that the mass formulae would scatter about the real masses, such that an average would yield reasonable values. The mass formula of Pape and Antony [Pap88] did not calculate masses for this region and the mass table of Wapscra er al. [Wap88] was a compilation of known masses with an occasional extrapolated mass. Neither of these two were used in the average calculations. Occasionally, the $Q_{\beta}$ - value calculated with the mass formula of Comay et al. [Com88] was $1 / 2$ of all the other values calculate and would be excluded from the average. Table 6.1 shows the comparison of the Q. values measured in this research and the average values calculated using the mass tables [H: a88]. The errors listed in Table 6.1 indicate that averagiig the $Q_{\beta}$ - values calculated from the mass

Table 6.1. Comparison of measured $Q \beta$ - values with theoretical values : calculated using the nuclear mass tables [Hau 88 and references therein].

\begin{tabular}{|c|c|c|c|}
\hline Nuclide & $\begin{array}{c}\text { Experimental } \mathrm{Q}_{\beta}- \\
\text { Value (MeV) }\end{array}$ & $\begin{array}{l}\text { Ave. Theoretical } \\
\text { Q. Valuea (MeV) }\end{array}$ & $\begin{array}{c}\text { Error (Theory - Exp.) } \\
\text { (MeV) }\end{array}$ \\
\hline${ }^{168} \mathrm{Ho}$ & $2.93(3)$ & 2.97 [9] & $0.04(3)$ \\
\hline${ }^{169} \mathrm{Dy}$ & $3.2(3)$ & 3.19 [9] & $-0.01(30)$ \\
\hline${ }^{171} \mathrm{Ho}$ & $3.2(6)$ & $3.16[9]$ & $-0.04(60)$ \\
\hline $174 \mathrm{Er}$ & $1.8(2)$ & $1-^{-+}[8]^{b}$ & $-0.09(20)$ \\
\hline
\end{tabular}

a Repored value is an average of the calculated Qq. values. The number of mass tables used in the calculation is in [ ].

b The Q8-value calculated using the mass table in reference [Com88] was not used in determining the average for this isotope due to it's large discrepancy with the other values calculated. 
tables works well for this region.

The production of more undiscovered neutron-rich rare-earth nuclides by the methods of this research is not a very likely proposition. The actual experimental time required for identifying the new isotopes ${ }^{169} \mathrm{Dy}$ and ${ }^{174} \mathrm{Er}$ was approximately 3 days running time for each. Due to the fact that the cross sections drop off by an order of magnitude for each neutron added, producing new Dy, $\mathrm{Ho}$, and Er isotopes would require -30 days of experinents at similar source intensities. Since the most neutron-rich isotopes of $\mathrm{Er}$ and $\mathrm{Xb}$ were already used for this research, no gain can be obtained by changing the target/projectile selection. Increases in the beam intensity could help; however, the SuperHILAC was running extremely well during the ${ }^{170}$ F.r beam experiments, and probably only a factor of two increase could be obrained. Performing this research at the UNILAC accelerator in GSI would allow use of $11.4 \mathrm{MeV} / \mathrm{u}$ ion beams, with similar intensities as those from the SuperHIIAC. This increase in team energy w'ould allow for more than twice the target material to be traversed before the ion beam energy dropped below the Coulomb barrier. With both an increase in the target thickness, due to increased beam energy, and the beam intensity, the new isotopes might be extended one more neutron in this region by multinucleon transfer reactions.

Production of these isotopes by other reaction mechanisms might be a more feasible possibility. Production of actinium and thorium isotopes using proton spallation reactions on ${ }^{238} \mathrm{U}$ yielded strong sources [Chu83]. Cioss-sections were calculated in this reseanch for ${ }^{231} \mathrm{Ac},{ }^{233} \mathrm{Ac},{ }^{231} \mathrm{Th}$, and ${ }^{233} \mathrm{Th}$. The same isotopes were produced in multinucleon transfer reactions of ${ }^{238} \mathrm{U}$ on natw targets at GSI [Gip86]. The cross-sections for ${ }^{231} \mathrm{Ac},{ }^{233} \mathrm{Ac},{ }^{231} \mathrm{Th}$, and ${ }^{233} \mathrm{Th}$ in the GSI study were approximately the same as those found in the proton spallation of ${ }^{238} \mathrm{U}$. 
However, due to differences in the beams used, protons at $28 \mathrm{GcV} / \mathrm{u}$ versus $238 \mathrm{U}$ at $11.4 \mathrm{MeV} / \mathrm{u}$, much more target material was subtended by the protons $(\sim 2$ to 10 times thicker targets were used). Also, proton beams are easier to accelerate than heavy-ion beams, so 100 times more intense beams were used. Both of these factors resulted much higher yields, of the order of $10^{3}$ times more, from spallation of uranium with protons than from muldinucleon transfer reactions of ${ }^{238} \mathrm{U}$ projectiles on narW targets [Gip86]. There is a drawback to the proton spallation reactions in that most of the recoils stop in the target. Chemical separations of the uranium targets used in the proton spallation reactions [Chu83] were performed approximately every eight minutes to separate the actinium and thorium isotopes from the uranium. 


\section{APPENDIX A}

\section{COMPUTER CODE FOR ANALYSIS OF BETA SPECTRA}

Variables involving 'SPEC' in their title deal with the binary file saving format also used when storing other spectra generated in this study.

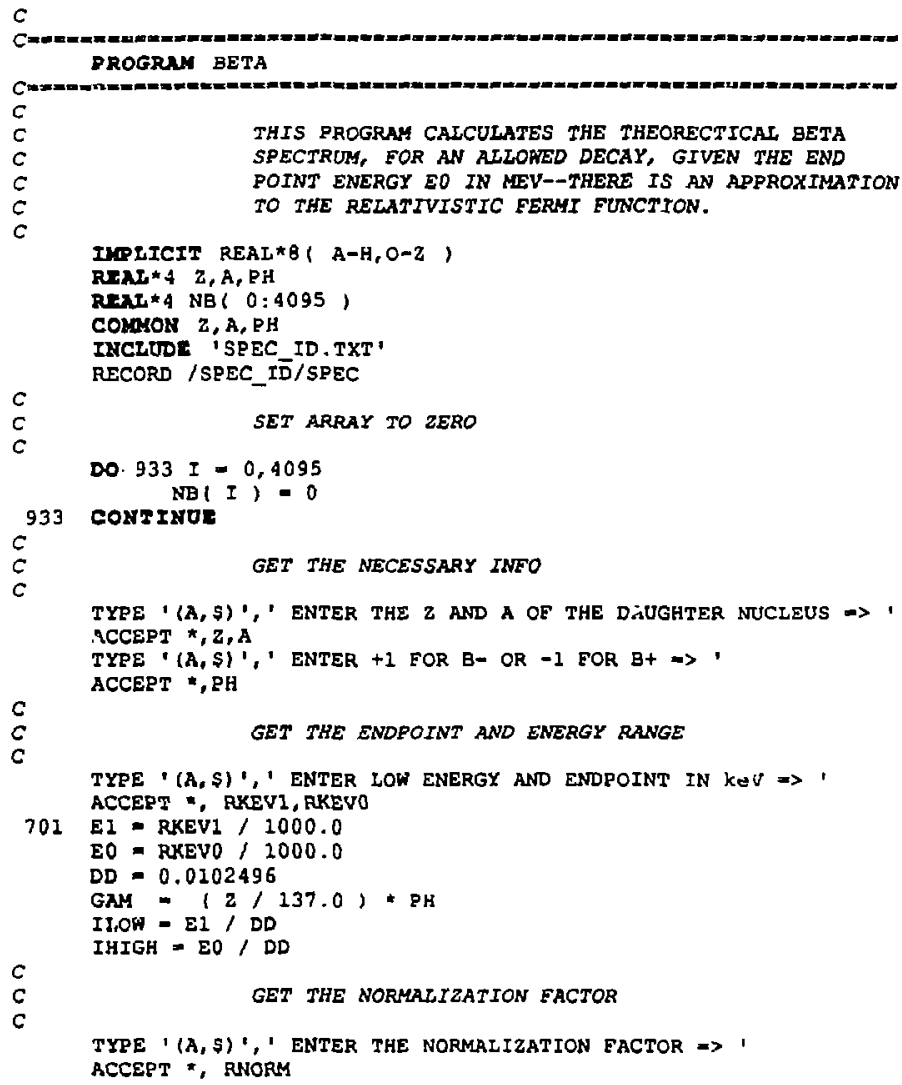




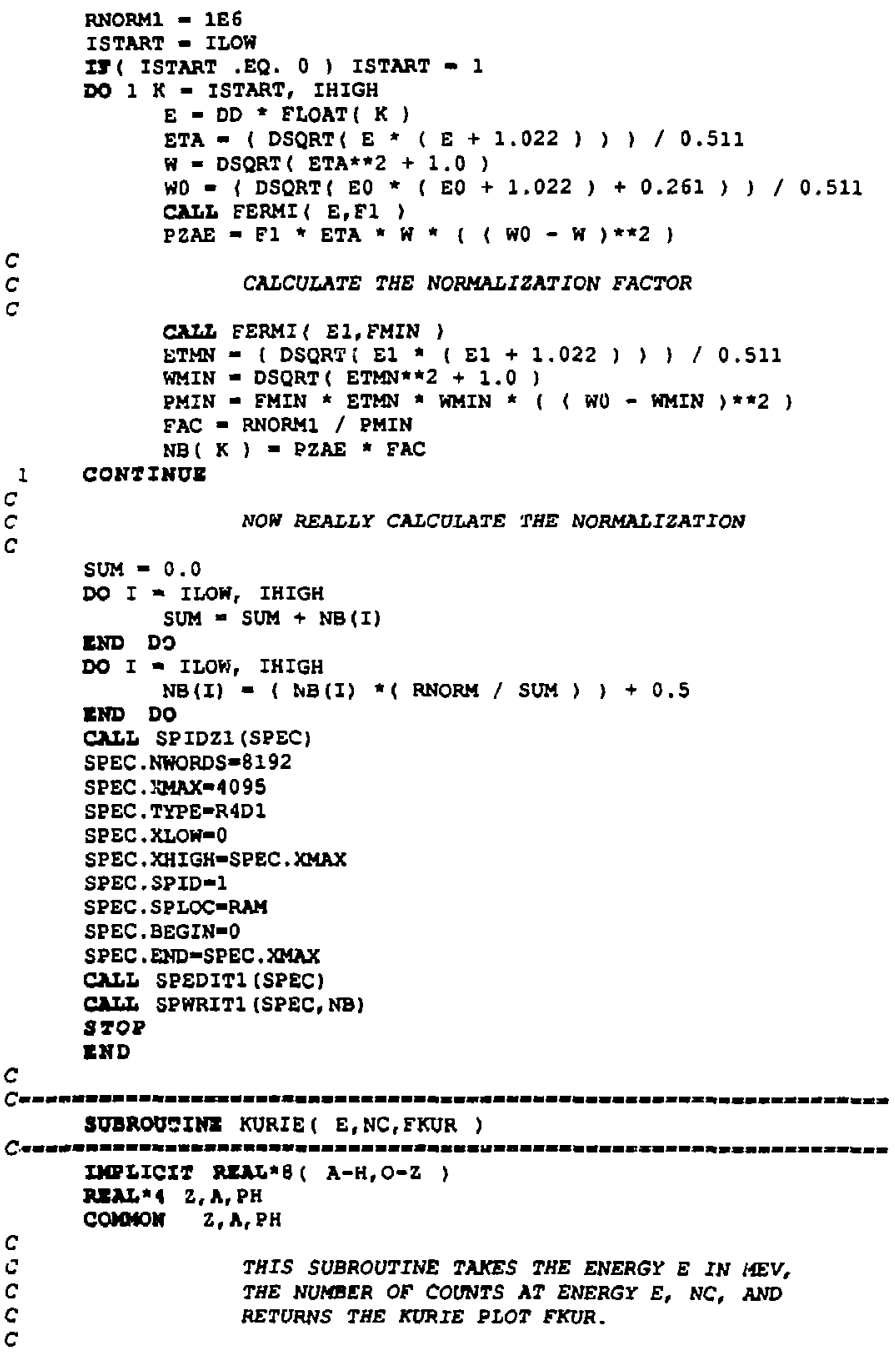




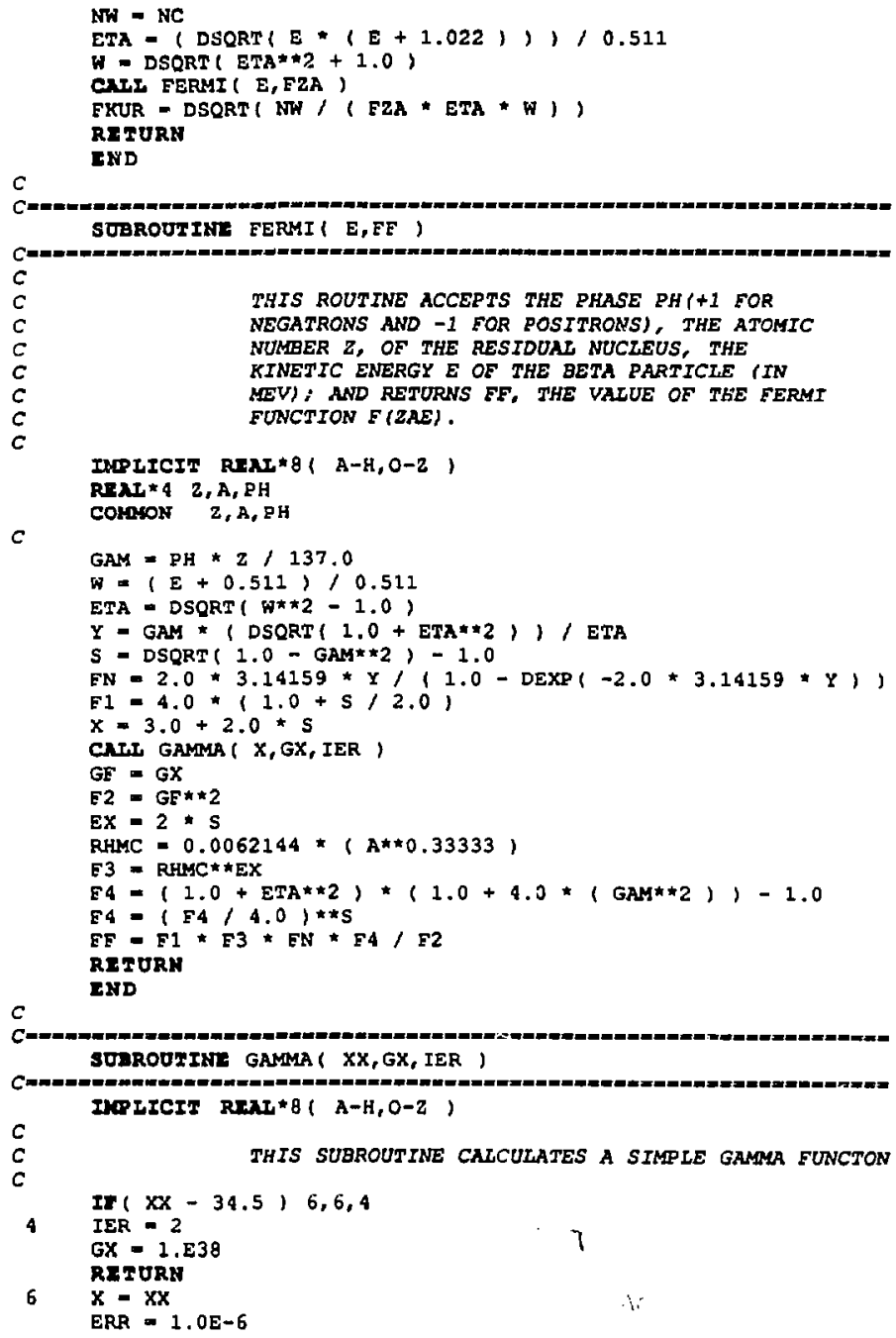

THIS SUBROUTINE CALCULATES A SIMPLE GAMRA FUNCTON 


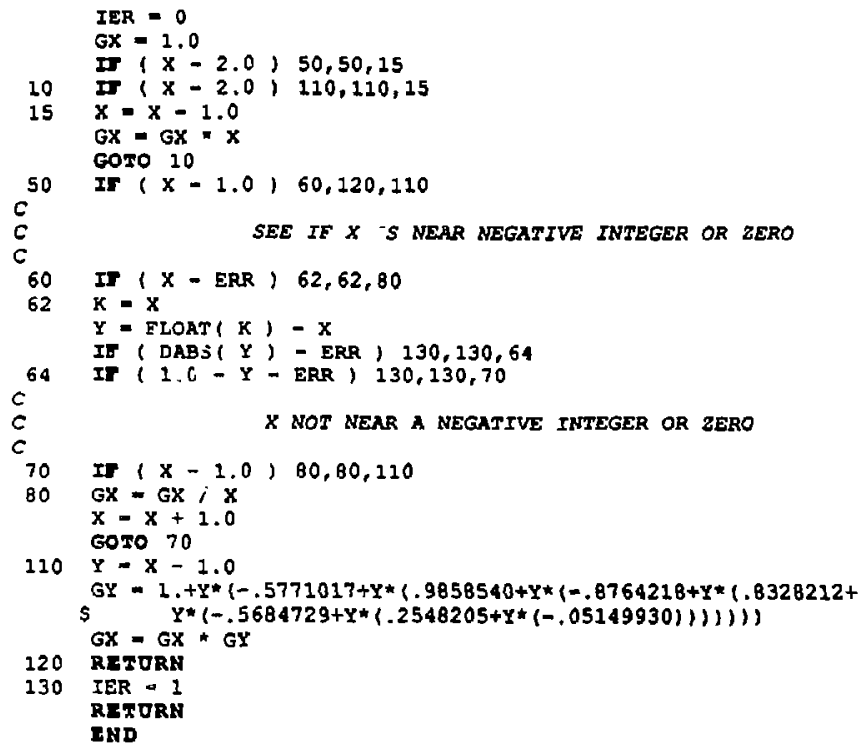




\section{APPENDIX B}

\section{MAXTMUM LIKELIHOOD COMPUTER CODE}

On-line data measured in this research contained timing information with resolution equal to the tape cycle time divided by $4 \mathrm{k}$ (a 4096-channel LeCroy scaler was used). Time binning this data and using a least-squares decay fitting program does not extract all possible informaton. A maximum likelihood code was written to determine half-lives from these data. The code used assumes only one radioactive substance is being measured and no background radiations. The probability for observing decay between time $t$ and $t+d r$ during measuring interval $T$ is:

$$
G(\lambda, t) d t=\lambda e^{-\lambda t}\left(1 \cdot e^{-\lambda T}\right)^{-1} d t
$$

Now, the probability for observing $M$ decays at imes $t_{1}, t_{2}, \ldots, t_{M}$, is proportional to:

$$
G\left(\lambda, t_{1}, t_{2}, \ldots, t_{M}\right)=\lambda^{M} \prod_{i=1}^{M} e^{-\lambda t_{i}}\left(1-e^{-\lambda T}\right)^{-1}
$$

The probability, $\mathrm{G}$, is a function of $\lambda$, and the procedure involves finding the value of $\lambda$ which maximizes $G(\lambda)$ [or $\ln (G(\lambda))]$ :

$$
\ln \left(G\left(\lambda, t_{1}, t_{2}, \ldots, t_{M}\right)\right)=-M \ln \left(1-e^{-\lambda T}\right)+M \ln \lambda \cdot \sum_{i=1}^{M} \lambda t_{i}
$$

Using the relation between $\lambda$ and $t_{1 / 2}, \lambda=\ln (2) / t_{1 / 2}, \ln \left(G\left(t_{1 / 2}\right)\right)$ was calculated by the code listed below. The output file was examined to determine the channel, or time, with the maximum probability. This tirne value corresponded to the maximum 
likelihood half-life. A simple error procedure was used where the probability histogram was examined to ascertain the two time values where the probability dropped to $50 \%$ of the maximum value. The range of these two values has been found to correspond to $68 \%$ confidence limits [Lyo86]. However, since the in of the probability was calculated, one looked to determine where the probability decreased by 0.693 , i.e., $-\ln (1 / 2)$.

Variables involving 'SPEC' in their tide deal with the binary file saving format also used when storing other spectra generated in this study. Formulae (1-3) above and the full details of their derivation can be found in Segre [Seg77].

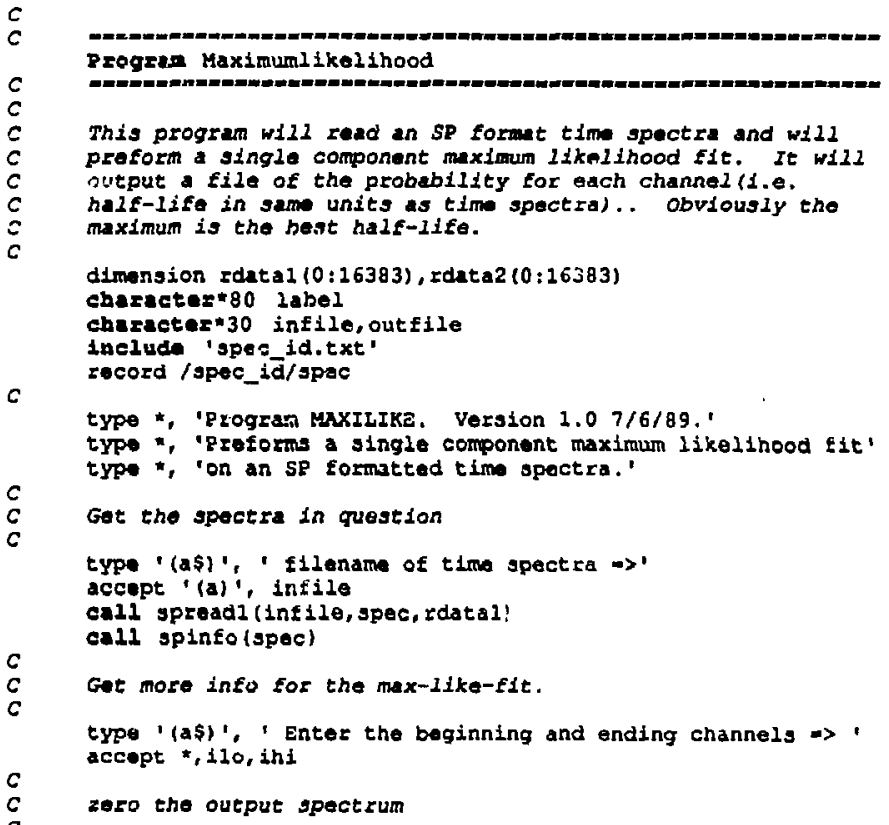




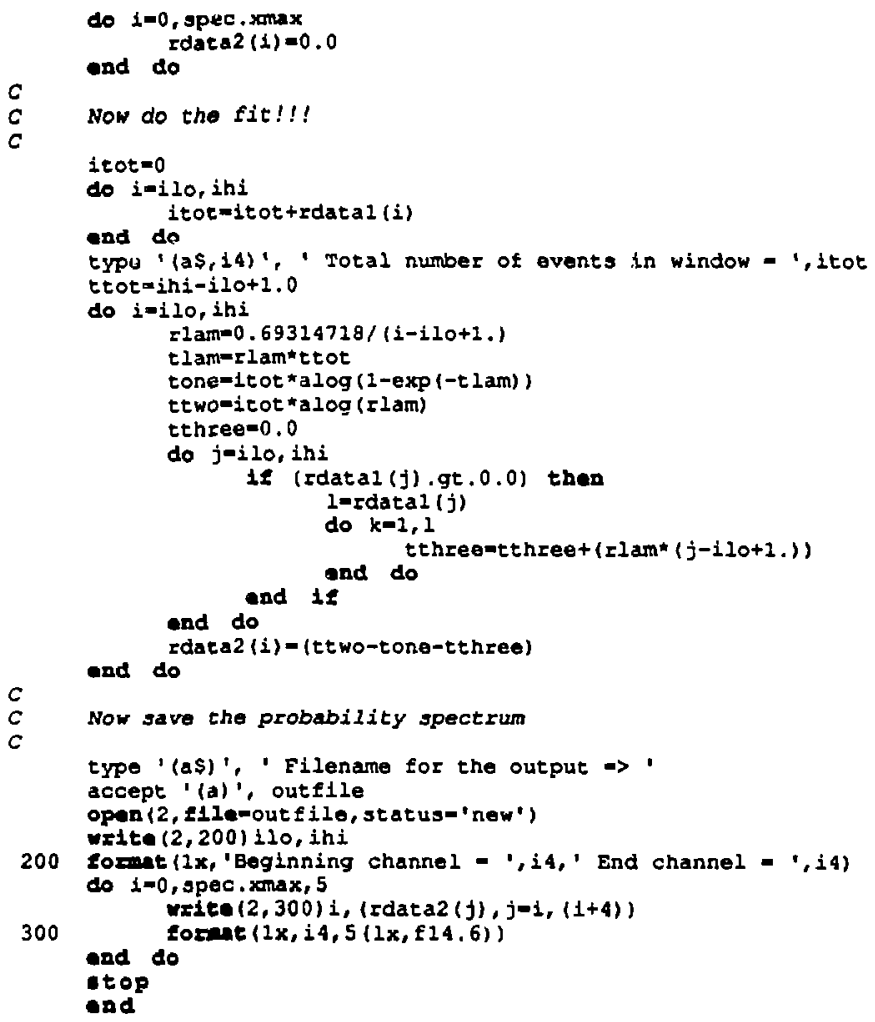




\section{REFERENCES}

[Avi81] F.T. Avigrone, H. Noma, D.M. Moltu, and K.S. Toth, Nucl. Instrum. \& Methods 189, 453 (1981).

[Bel86] R.A. Belshe and M.K. Lee, Nucl. Instrum. \& Methods in Physics Research A253, 65 (1986).

[Bel87a] R.A. Belshe, EVA Reference Manual, Engineering Division PUB-3062, Lawrence Berkeley Laboratory (198?).

[Bel87b] R.A. Belshe, SUSEE Reference Manual, Engineering Division PUB3061 Rev., Lawrence Berkeley Laboratory (1987).

[Bla52] J.M. Blatt and V.F. Weisskopf, Theoretical Nuclear Physics, (John Wiley and Sons, New York, 1952) p.627.

[Bos88] U. Bosch, W.-D. Schrmidt-Oth, E. Runte, P. Tidemand-Petersson, P. Koschel, F. Meissner, R. Kirchner, O. Klepper, E. Roeckl, K Rykaczewski, and D. Schardt, Nuch. Phys. A477, 89 (1988).

[Bro84] E. Browne, Nucl. Data Sheets 41, 511 (1984).

[Bro86] E. Browne and R.B. Firestone, Table of Radioactive Isoropes, (Joinn Wiley \& Sons, Inc., 1965).

[Cha89] R.M. Chasteler, J.M. Nitschks, R.B. Firestone, K.S. Vierinen, P.A. Wilmarth, and A.A. Shihab-Eldin, Z. Phys. A 332, 239 (1989).

[Chu83] Y.Y. Chu and M.L. Zhou, Phys. Rev. C 28, 1379 (1983).

[Com88] E. Comay, I. Kelson, and A. Zidon, At. Data Nucl. Data Tables 39, 235 (1988).

[Cow70] G.A. Cowan, "Heavy Element Synthesis by Prompt Multiple Neutron Caprure", in Proceedings of The Robert A. Welch Foundation 
Conferences on Chemical Research, XIII. The Transuraniurs Eleinents The Mendeleev Centennial, edited by W.O. Milligan (Houston, Texas, 1970) p. 291.

[Dus88] G. Dussel, E. Caurier, and A.P. Zuker, At. Data Nucl. Data Tables 39. 205 (1988).

[Geh82] R.J. Gehrke, R.C. Greenwood, J.D. Baker, and D.H. Meikrantz, Z. Phys. A 306, 363 (1982).

[Gip86] K.-L. Gippert, E. Runte, W.-D. Schmidt-Ott, P. Tidemand-Petersson, N. Kaffrell, F. Peuser, R. Kirchuer, O. KJepper, W. Kuncewicz, P.O. Larsson, E. Roeckl, D. Schardt, and K. Rykaczewski, Nucl. Phys. A453, 1 (1986).

[Gra84] R.L. Graham, J.S. Geiger, and M.W. Johns, Can. J. Phys. 50, 513 (1972).

[Gre85] K.E. Gregorich, Actinide Production in ${ }^{136} \mathrm{Xe}$ Bombardments of ${ }^{249} \mathrm{Cf}$, Ph.D. Thesis, LBL-20192 (1985).

[Gre87] R.C. Greenwood, R.A. Anderl, J.D. Cole, and H. Willmes, Phys. Rev. C 35, 1965 (1987).

[HauTi] P.E. Haustein and A.B. Tucker, Nucl. Phys. A173, 321 (1971).

[Hau88] P.E. Haustein, At. Data Nucl. Data Tables 39, 185 (1988). Nuclear nass tables referenced therein: [Pap88], [Dus88], [M81882], [Mul88b], [Com:s8], [Sat88], [Tac88], [Spa88], [Jăn88], [Mas88], and [Wap88].

[Hub80] F. Hubert, A. Fleury, R. Bimbol, and D. Gardes, Supplement aux Annules de Physique 5, 1 (1980).

[Hyd71] E.R. Hyde, The Nuclear Properties of the Heavy Elements III, Fission Properties, (Dover Publications, Inc., New York, 197!) p. 128. 
[Jăn88] J. Jänecke and P.J. Masson, At. Data Nucl. Data Tables 39, 265 (1988).

[Kat78] R. Katajanheimo, T. Tuumala, and E. Hammaren, Z. Phys. A 286, 57 (1978)

[Kaw73] K. Kawade, H. Yamamoto, K. Tsuchiya and T. Katoh, J. Phys. Soc. Jpn 34, 857 (1973).

[Kaw88] Y. Kawase and K. Okano, Z. Phys. A 330, 231 (1988).

[Kla84] H.V. Klapdor, J. Metzinger, and T. Oda, At. Data Nucl. Data Tables 31, 81 (1984).

[Kra79] J.V. Kratz, W. Brulchle, G. Franz, M. Schädel, J. Warnecke, G. Wirth, and M. Weis, Nucl. Phys. A332, 477 (1979).

[Led78] Table of Isotopes, 7th Edition, edited by C.M. Lederer and V.S. Shirely (John Wiley \& Sons, Inc., New York, 1978).

[Lyo86] L. Lyons, Statistics for nuclear and particle physicists, (Cambridge University Press, Cambridge, 1986) p. 88-90.

[Mac86] H. Mach, A. Piorowski, R.L. Gill, R.F. Casten, and D.D. Wamer, Phys. Rev. Lett. 56, 1547 (1986).

[Mas88] P.J. Masson and J. Jănecke, At. Data Nucl. Data Tables 39, 273 (1988).

[Mei89] F. Meissner, K. Becker, W.-D. Schmidt-Ott, U. Bosch, V. Kunze, H. Salewsti, E. Roeckl, H. Keller, R. Kirchner, O. Klepper, and D. Schard, GSI Scientific Repon 1988, ISSN 0174-0814, p. 32, (1989).

[Mbl81] P. Mbller and J.R. Nix, At. Data Nucl. Data Tables 26, 165 (1981).

[Mol88a] P. Moller and J.R. Nix, At. Data Nucl. Data Tables 39, 213 (1988).

[M8188b] P. Mbller, W.D. Myers, W.J. Swiatecki, and J. Treiner, At. Data Nucl. Data Tables 39, 225 (1988). 
[Mol89] P. Möller and J. Randrup, Lawrence Berkeley Laboratory Report No. 27504 (1989), to be published in Nucl. Phys. A.

[Mor81] L.G. Moretto and R.P. Schmitt, Kep. Prog. Phys. 44, 533 (1981).

[Nil69] S.G. Nilsson, C.F. Tsang, A. Sobiczewski, Z. Szymanski, S. Wycech, C. Gustafson, I.-L. Lamm, P. Moller, and B. Nilsson, Nucl. Phys. A131, 1 (1969).

[Nit83] J.M. Nitschke, Nucl. Instum. \& Methods 206, 341 (1983).

[One72] R.A. O'Neil and D.G. Burke, Nucl. Phys. A195, 207 (1972).

[Pag72] L. Pages, E. Bertel, H Joffre, and L. Sklavenities, Atomic Data 4, 1 (1972).

[Pap88] A. Pape and M.S. Antony, At. Data Nucl. Data Tables 39, 201 (1988).

[Ros78] F. Rosel, H.M. Fries, K. Adler, and H.C. Pauli, At. Data Nucl. Data Tables 21, 91 (1978).

[Rou69] J.T. Routti and S.G. Prussin, Nucl. Instrum. \& Methods 72, 125 (1969).

[Run85] E. Runte, K.-L. Gippert, W.-D. Schmidt-Ott, P. Tidemand-Petersson, L. Ziegeler, R. Kirchner, O. Klepper, P. O. Larsson, E. Roeckl, D. Schardt, N. Kaffrell, P. Peuser, M. Bemas, P. Dessagne, M. Langevin, and K. Rykaczewski, Nucl. Phys. A441, 237 (1985).

[Ryk84] K. Rykaczewski, P.O. Larsson, R. Kirchner, O.Klepper, E. Roeckl, D. Schardt, K.L. Gippert, E. Runte, W.-D. Schmidt-Ott, P. TidemandPetersson, N. Kaffrell, P. Peuser, W. Kurcewicz and J. Zylicz, Institut für Kernchemie Universităt Mainz, Jahresbericht 1983, IKMz 84-1, p. 72, (1984). 
[Ryk88] K. Rykaczewski, K.-L. Gipper, N. Kaffrell, R. Kirchner, O. Klepper, V.T. Koslowsky, W. Kurcewicz, W. Nazarewicz, E. Roeckl, E. Runte, D. Schardt, W.-D. Schmidt-Ort, and P. Tidemand-Petersson, GSI Scientific Report 1987, ISSN 0174-0814, p. 30 (1988).

[Ryk89] K. Rykaczewski, K.-L. Gipper, N. Kaffrell, R. Kirchner, O. Klepper, V.T. Koslowsky, W. Kurcewicz, W. Nazarewicz, E. Roeckl, E. Runte, D. Schardt, W.-D. Schmidt-Ort, and P. Tidemand-Petersson, Nucl. Phys. A499, 529 (1989).

[Sat88] L. Sarpathy and R.C. Nayak, At. Data Nucl. Data Tables 39, 241 (1988).

[Sch76] W.U. Schrtyder, J.R. Birkelund, J.R. Huizenga, K.L. Wolf, J.R. Unik, and V.E. Viola, Jr., Phys. Rev. Lett. 36, 514 (1976).

[Sch77a] W.U. Schröder and J.R. Huizenga, Ann. Rev. Nucl. Sci. 27, 465 (1977).

[Sch77b] W.U. Schroder, J.R. Birkelund, J.R. Huizenga, K.L. Wolf, and V.E. Viola, Jr., Phys. Rev. C 16, 623 (1977).

[Seg77] E. Segre, Nuclei and Particles, Second Edition, (W.A. Benjamin, Inc., Massachusetts, 1977) p. 193.

[Shi84] V.S. Shirley, Nucl. Data Sheets 43, 127 (1984).

[Spa88] L. Sparier and S.A.E. Johansson, At. Data Nucl. Data Tables 39, 259 (1988).

[Tac88] T. Tachibana, M. Uno, M. Yamada, and S. Yamada, At. Data Nucl. Data Tables 39, 251 (1988).

[Tak61] K. Takahashi, T. Kuroyanagi, H. Yuta, K. Kotajima, K. Nagatani, and H. Morinaga, J. Phys. Soc. Jpn 16, 1664 (1961). 
[Tak73] K. Takahashi, M. Yamada, and T. Kondoh, At. Data Nucl. Data Tables 12, 101 (1973).

[Tak88] K. Takahashi, privare communication (1988).

[Tuu78] T. Tuumala, R. Katajanheimo, and O. Heinonen, Phys. Scripta 18, 31 (1978).

[Val62] J. Valentin, D.J. Horen, and J.M. Hollander, Nucl. Phys. 31, 373 (1962).

[Van76] R. Vandenbosch, M.P. Webb, and T.D. Thomas, Phys. Rev, Lett. 36. 459 (1976).

[Wap88] A.H. Wapstra, G. Audi, and R. Hoekstra, At. Data Nucl. Data Tables 39. 281 (1988).

[Wil60] R.G. Wille and R.W. Fink, Phys. Rev. 118, 242 (1960).

[Wil80] W.W. Wilcke, J.R. Birkelund, H.J. Wollersheim, A.D. Hoover, J.R. Huiznega, W.U. Schrbder, and L.E. Tubbs, At. Data Nucl. Data Tables 25, 389 (1980).

[Wil88] P.A. Wilmarth, Beta-Delayed Proton Emission in Neutron-Deficient Lanthanide Isotopes, Ph.D. Thesis, LBL-26101 (1988).

[Wol84] H.-J. Wollersheim, GSI Preprint GSI-84-34 (1984).

[Zyc83] 1. Zychor, K. Rykaczewski, H. Ahrens, H. Folger, W. Kurcewicz, K. Summerer, N. Kaffrell, and N. Trautmann, Radiochim. Acta 33, 1 (1983). 